\title{
The Decarboxylative Strecker Reaction
}

\author{
Deepankar Das, Matthew T. Richers, Longle Ma and Daniel Seidel* \\ Department of Chemistry and Chemical Biology \\ Rutgers, The State University of New Jersey, Piscataway, New Jersey 08854 (USA)
}

\section{Supporting Information}

General Information: The amino acids were purchased from commercial sources and used as received. Trimethylsilyl cyanide (TMSCN) and reagent grade $n$-butanol were purchased from Sigma-Aldrich and used as received. The aldehydes were purified either by distillation or by recrystallization prior to use. Ethyl glyoxalate solution ( $\sim 50 \%$ in toluene) was freshly distilled under nitrogen prior to use. Microwave reactions were carried out in a CEM Discover reactor. Silicon carbide ( $\mathrm{SiC}$ ) passive heating elements were purchased from Anton Paar. Purification of reaction products was carried out by flash column chromatography using Sorbent Technologies Standard Grade silica gel (60 A, 230-400 mesh). Analytical thin layer chromatography was performed on EM Reagent $0.25 \mathrm{~mm}$ silica gel $60 \mathrm{~F}_{254}$ plates. Visualization was accomplished with UV light, potassium permanganate, and Dragendorff-Munier stains followed by heating. Melting points were recorded on a Thomas Hoover capillary melting point apparatus and are uncorrected. Infrared spectra were recorded on an ATI Mattson Genesis Series FT-Infrared spectrophotometer. Proton nuclear magnetic resonance spectra $\left({ }^{1} \mathrm{H}-\mathrm{NMR}\right)$ were recorded on a Varian VNMRS-500 MHz and are reported in ppm using the solvent as an internal standard $\left(\mathrm{CDCl}_{3}\right.$ at $\left.7.26 \mathrm{ppm}\right)$. Data are reported as app $=$ apparent, $\mathrm{s}=$ singlet, $\mathrm{d}=$ doublet, $\mathrm{t}=$ triplet, $\mathrm{q}=$ quartet, $\mathrm{m}=$ multiplet, comp = complex, br = broad; and coupling constant(s) in Hz. Proton-

decoupled carbon nuclear magnetic resonance spectra $\left({ }^{13} \mathrm{C}-\mathrm{NMR}\right)$ were recorded on a Varian VNMRS-500 $\mathrm{MHz}$ and are reported in ppm using the solvent as an internal standard $\left(\mathrm{CDCl}_{3}\right.$ at $77.0 \mathrm{ppm}$ ). Mass spectra were recorded on a Finnigan LCQ-DUO mass spectrometer. Products $\mathbf{2 a},{ }^{1} \mathbf{4 a},{ }^{2} \mathbf{5},{ }^{3} \mathbf{1 0},{ }^{4} \mathbf{1 2},{ }^{5} \mathbf{1 4},{ }^{6}$ and $\mathbf{1 5}{ }^{7}$ were previously reported and their published characterization data matched our own in all respects.

General Procedure: A $10 \mathrm{~mL}$ microwave reaction tube was charged with a $10 \times 8 \mathrm{~mm} \mathrm{SiC}$ passive heating element, amino acid $(1.3 \mathrm{mmol}), n$-BuOH $(2 \mathrm{~mL})$, aldehyde $(1 \mathrm{mmol})$ and TMSCN (1.2 mmol). The reaction tube was sealed with a Teflon-lined snap cap, and heated in a microwave reactor at $200{ }^{\circ} \mathrm{C}(200 \mathrm{~W}, 70-150 \mathrm{psi})$ for 10 minutes (Note: SiC passive heating elements must not be used in conjunction with stir bars for they may score glass and cause vessel failure). After cooling with compressed air flow, the reaction mixture was transferred to a round bottom flask and the vessel was rinsed with EtOAc $(4 \times 2 \mathrm{~mL})$. Solvent was then removed in vacuo and the reaction mixture was loaded onto a short column and purified by silica gel chromatography. Note: Due to the use of TMSCN and the potential for HCN formation, all operations should be conducted inside a well ventilated fume hood. 
1-(2-Chlorobenzyl)pyrrolidine-2-carbonitrile (4b): Following the general procedure<smiles>N#CC1CCCN1Cc1ccccc1Cl</smiles>
compound $4 \mathbf{b}$ was obtained from $L$-proline and $o$-chlorobenzaldehyde as a colorless liquid in 97\% yield $\left(\mathrm{R}_{\mathrm{f}}=0.17\right.$ in hexanes/EtOAc 95:5 v/v); IR $(\mathrm{KBr})$ 2960, 2815, 2221, 1572, 1474, 1444, 1376, 1335, 1248, 1138, 1052, 1039, 882, $755 \mathrm{~cm}^{-1}$; ${ }^{1} \mathrm{H}$ NMR $\left(500 \mathrm{MHz}, \mathrm{CDCl}_{3}\right) 7.43$ (app dd, $\left.J=7.2,2.0 \mathrm{~Hz}, 1 \mathrm{H}\right), 7.38-$ 7.34 (m, 1H), 7.27-7.19 (comp, 2H), 3.98 (d, $J=13.8 \mathrm{~Hz}, 1 \mathrm{H}), 3.86$ (d, $J=13.8$ $\mathrm{Hz}, 1 \mathrm{H}), 3.77$ (dd, $J=7.4,2.6 \mathrm{~Hz}, 1 \mathrm{H}), 2.95-2.88(\mathrm{~m}, 1 \mathrm{H}), 2.70-2.62(\mathrm{~m}, 1 \mathrm{H})$, 2.24-2.09 (comp, 2H), 2.02-1.84 (comp, 2H); ${ }^{13} \mathrm{C}$ NMR (125 MHz, $\left.\mathrm{CDCl}_{3}\right) \delta 135.3,134.3$, $130.5,129.7,128.7,126.7,118.3,53.6,53.5,51.1,29.7,22.0 ; m / z(E S I-M S) ~ 194.2 ~[M-C N]^{+}$.

1-(3-Chlorobenzyl)pyrrolidine-2-carbonitrile (4c): Following the general procedure compound<smiles>N#CC1CCCN1Cc1cccc(Cl)c1</smiles>
4c was obtained from $L$-proline and $m$-chlorobenzaldehyde as a colorless liquid in $89 \%$ yield $\left(\mathrm{R}_{\mathrm{f}}=0.14\right.$ in hexanes/EtOAc 95:5 v/v); IR $(\mathrm{KBr}) 3062$, 2961, 2881, 2820, 2222, 1600, 1576, 1475, 1431, 1373, 1334, 1210, 1144, 1076, 995, 883, 786, 685; ${ }^{1} \mathrm{H}$ NMR $\left(500 \mathrm{MHz}, \mathrm{CDCl}_{3}\right) 7.36$ (s, 1H), 7.277.20 (comp, 3H), 3.87 (d, $J=13.3 \mathrm{~Hz}, 1 \mathrm{H}), 3.71(\mathrm{dd}, J=7.5,2.4 \mathrm{~Hz}, 1 \mathrm{H})$, $3.65(\mathrm{~d}, J=13.2 \mathrm{~Hz}, 1 \mathrm{H}), 2.92(\mathrm{ddd}, J=12.7,8.5,4.2 \mathrm{~Hz}, 1 \mathrm{H}), 2.61-2.52$ (m, 1H), 2.23-2.07 (comp, 2H), 2.01-1.87 (comp, 2H); $\left.{ }^{13} \mathrm{C} \mathrm{NMR} \mathrm{(125} \mathrm{MHz,} \mathrm{CDCl}_{3}\right) \delta 139.7$, $134.3,129.7,128.6,127.6,126.8,117.7,55.8,53.2,51.1,29.4,21.8 ; \mathrm{m} / z$ (ESI-MS) 194.1 [M$\mathrm{CN}]^{+}$.

1-(4-Chlorobenzyl)pyrrolidine-2-carbonitrile (4d): Following the general procedure<smiles>N#CC1CCCN1Cc1ccc(Cl)cc1</smiles>
compound 4d was obtained from $L$-proline and $p$-chlorobenzaldehyde as a white sticky solid in $97 \%$ yield $\left(\mathrm{R}_{\mathrm{f}}=0.13\right.$ in hexanes/EtOAc 95:5 v/v); IR (KBr) 2960, 2819, 2222, 1644, 1491, 1447, 1409, 1376, 1334, 1124, 1084, 1016, 881, $840 \mathrm{~cm}^{-1}$; ${ }^{1} \mathrm{H}$ NMR (500 MHz, $\mathrm{CDCl}_{3}$ ) 7.29 (comp, 4H), 3.87 $(\mathrm{d}, J=13.2 \mathrm{~Hz}, 1 \mathrm{H}), 3.67(\mathrm{dd}, J=7.4,2.2 \mathrm{~Hz}, 1 \mathrm{H}), 3.63(\mathrm{~d}, J=13.2 \mathrm{~Hz}$, $1 \mathrm{H}), 2.94-2.86(\mathrm{~m}, 1 \mathrm{H}), 2.59-2.51(\mathrm{~m}, 1 \mathrm{H}), 2.20-2.07$ (comp, 2H), 2.001.84 (comp, 2H); ${ }^{13} \mathrm{C}$ NMR $\left(125 \mathrm{MHz}, \mathrm{CDCl}_{3}\right) \delta 136.1,133.1,130.0,128.6,117.7,55.7,53.1$, $51.1,29.4,21.8 ; m / z$ (ESI-MS) $194.1[\mathrm{M}-\mathrm{CN}]^{+}$.

1-(2-Methylbenzyl)pyrrolidine-2-carbonitrile (4e): Following the general procedure<smiles>Cc1ccccc1CN1CCCC1C#N</smiles>
compound $4 \mathbf{e}$ was obtained from $L$-proline and $o$-tolualdehyde as a colorless liquid in $95 \%$ yield $\left(\mathrm{R}_{\mathrm{f}}=0.21\right.$ in hexanes/EtOAc 95:5 v/v); IR $(\mathrm{KBr}) 3018$, 2957, 2882, 2813, 2221, 1693, 1494, 1460, 1375, 1334, 1286, 1184, 1125, 1051, 882, $745 \mathrm{~cm}^{-1}$; ${ }^{1} \mathrm{H}$ NMR $\left(500 \mathrm{MHz}, \mathrm{CDCl}_{3}\right) 7.33$ (app d, $\left.J=7.4 \mathrm{~Hz}, 1 \mathrm{H}\right)$, $7.23-7.15$ (comp, 3H), 3.93 (d, $J=13.0 \mathrm{~Hz}, 1 \mathrm{H}), 3.67$ (d, $J=12.9 \mathrm{~Hz}, 1 \mathrm{H})$, 3.68 (dd, $J=7.3,2.9 \mathrm{~Hz}, 1 \mathrm{H}), 2.91$ (ddd, $J=12.7,8.4,4.3 \mathrm{~Hz}, 1 \mathrm{H}), 2.65-2.58$ (m, 1H), 2.40 (s, 3H), 2.21-2.08 (comp, 2H), 2.01-1.85 (comp, 2H); ${ }^{13} \mathrm{C} \mathrm{NMR} \mathrm{(125} \mathrm{MHz,}$ $\left.\mathrm{CDCl}_{3}\right) \delta 137.3,135.6,130.3,129.3,127.4,125.7,118.1,54.4,53.2,51.0,29.5,21.8,18.9 ; \mathrm{m} / \mathrm{z}$ (ESI-MS) $174.1[\mathrm{M}-\mathrm{CN}]^{+}$. 
1-(3-Methylbenzyl)pyrrolidine-2-carbonitrile (4f): Following the general procedure compound<smiles>Cc1cccc(CN2CCCC2C#N)c1</smiles>
$4 f$ was obtained from $L$-proline and $m$-tolualdehyde as a colorless liquid in $92 \%$ yield $\left(\mathrm{R}_{\mathrm{f}}=0.14\right.$ in hexanes/EtOAc 95:5 v/v); IR $(\mathrm{KBr}) 2959,2922$, 2881, 2814, 2221, 1610, 1487, 1460, 1378, 1334, 1160, 1124, 1089, 886, 789, $700 \mathrm{~cm}^{-1} ;{ }^{1} \mathrm{H}$ NMR $\left(500 \mathrm{MHz}, \mathrm{CDCl}_{3}\right) 7.23(\mathrm{~m}, 1 \mathrm{H}), 7.20-7.15$ (comp, 2H), 7.10 (app d, $J=7.4 \mathrm{~Hz}, 1 \mathrm{H}), 3.90(\mathrm{~d}, J=12.9 \mathrm{~Hz}, 1 \mathrm{H}), 3.71$ $(\mathrm{dd}, J=7.4,2.4 \mathrm{~Hz}, 1 \mathrm{H}), 3.64(\mathrm{~d}, J=12.9 \mathrm{~Hz}, 1 \mathrm{H}), 2.95$ (ddd, $J=12.7$, 8.5, 4.2 Hz, 1H), 2.63-2.56 (m, 1H), 2.36 (s, 3H), 2.25-2.07 (comp, 2H), 2.02-1.86 (comp, 2H); ${ }^{13} \mathrm{C}$ NMR $\left(125 \mathrm{MHz}, \mathrm{CDCl}_{3}\right) \delta 138.0,137.4,129.4,128.2,128.1,125.8,117.8,56.4,53.1,51.1$, 29.4, 21.7, 21.2; $\mathrm{m} / z(\mathrm{ESI}-\mathrm{MS}) 174.1[\mathrm{M}-\mathrm{CN}]^{+}$.

1-(4-Methylbenzyl)pyrrolidine-2-carbonitrile (4g): Following the general procedure<smiles>Cc1ccc(CN2CCCC2C#N)cc1</smiles>
compound $\mathbf{4 g}$ was obtained from $L$-proline and $p$-tolualdehyde as a colorless liquid in $93 \%$ yield $\left(\mathrm{R}_{\mathrm{f}}=0.14\right.$ in hexanes/EtOAc $\left.95: 5 \mathrm{v} / \mathrm{v}\right)$; IR (KBr) 2960, 2815, 2220, 1633, 1573, 1473, 1445, 1376, 1132, 1052, 1039, $755,683 \mathrm{~cm}^{-1}$; ${ }^{1} \mathrm{H}$ NMR $\left(500 \mathrm{MHz}, \mathrm{CDCl}_{3}\right) 7.26(\mathrm{~d}, J=7.9 \mathrm{~Hz}, 2 \mathrm{H}), 7.15$ $(\mathrm{d}, J=7.8 \mathrm{~Hz}, 2 \mathrm{H}), 3.89(\mathrm{~d}, J=12.9 \mathrm{~Hz}, 1 \mathrm{H}), 3.69(\mathrm{dd}, J=7.4,2.6 \mathrm{~Hz}$, $1 \mathrm{H}), 3.64(\mathrm{~d}, J=12.9 \mathrm{~Hz}, 1 \mathrm{H}), 2.94(\mathrm{ddd}, J=12.5,8.4,4.2 \mathrm{~Hz}, 1 \mathrm{H}), 2.62-$ 2.55 (m, 1H), 2.35 (s, 3H), 2.21-2.07 (comp, 2H), 2.01-1.85 (comp, 2H); ${ }^{13} \mathrm{C} \mathrm{NMR} \mathrm{(125} \mathrm{MHz,}$ $\left.\mathrm{CDCl}_{3}\right) \delta 137.0,134.4,129.1,128.7,117.9,56.1,53.0,51.1,29.4,21.8,21.0 ; \mathrm{m} / z$ (ESI-MS) $174.1[\mathrm{M}-\mathrm{CN}]^{+}$.

1-(4-Methoxybenzyl)pyrrolidine-2-carbonitrile (4h): Following the general procedure<smiles>COc1ccc(CN2CCCC2C#N)cc1</smiles>
compound $4 \mathbf{h}$ was obtained from $L$-proline and $p$-anisaldehyde as a colorless liquid in 92\% yield as a mixture of regioisomers; $\mathrm{rr}=4: 1\left(\mathrm{R}_{\mathrm{f}}=\right.$ 0.19 in hexanes/EtOAc 90:10 v/v); Characterization data of the major regioisomer: IR (KBr) 2958, 2816, 2222, 1612, 1513, 1462, 1377, 1301, 1245, 1174, 10393, $821 \mathrm{~cm}^{-1}$; ${ }^{1} \mathrm{H}$ NMR $\left(500 \mathrm{MHz}, \mathrm{CDCl}_{3}\right) 7.27$ (d, $J=$ $8.6 \mathrm{~Hz}, 2 \mathrm{H}), 6.86(\mathrm{~d}, J=8.6 \mathrm{~Hz}, 2 \mathrm{H}), 3.85(\mathrm{~d}, J=12.9 \mathrm{~Hz}, 1 \mathrm{H}), 3.79$ (s, $3 \mathrm{H}), 3.65(\mathrm{dd}, J=12.4,2.7 \mathrm{~Hz}, 1 \mathrm{H}), 3.59$ (d, $J=12.8 \mathrm{~Hz}, 1 \mathrm{H}), 2.91$ (ddd, $J=12.5,8.5,4.2 \mathrm{~Hz}$, 1H), 2.59-2.52 (m, 1H), 2.20-2.06 (comp, 2H), 2.00-1.82 (comp, 2H); ${ }^{13} \mathrm{C} \mathrm{NMR} \mathrm{(125} \mathrm{MHz,}$ $\left.\mathrm{CDCl}_{3}\right) \delta 158.9,129.9,129.6,117.9,113.7,55.7,55.1,52.9,51.0,29.3,21.7 ; \mathrm{m} / z$ (ESI-MS) $190.0[\mathrm{M}-\mathrm{CN}]^{+}$.

1-(4-Nitrobenzyl)pyrrolidine-2-carbonitrile (4i): Following the general procedure, but<smiles>N#CC1CCCN1Cc1ccc([N+](=O)[O-])cc1</smiles>
performing the reaction at $180^{\circ} \mathrm{C}$ for $30 \mathrm{~min}$, compound $4 \mathbf{i}$ was obtained from $L$-proline and $p$-nitrobenzaldehyde as an off-white solid in $80 \%$ yield $\left(\mathrm{R}_{\mathrm{f}}=0.24\right.$ in hexanes/EtOAc 80:20 v/v); mp: 80-83 ${ }^{\circ} \mathrm{C}$; IR $(\mathrm{KBr})$ 2961, 2820, 2220, 1606, 1518, 1346, 1108, 1015, 853, 806, $739 \mathrm{~cm}^{-1} ;{ }^{1} \mathrm{H}$ NMR $\left(500 \mathrm{MHz}, \mathrm{CDCl}_{3}\right) 8.17(\mathrm{~d}, J=8.4 \mathrm{~Hz}, 2 \mathrm{H}), 7.53(\mathrm{~d}, J=8.4 \mathrm{~Hz}$, $2 \mathrm{H}), 3.98(\mathrm{~d}, J=13.8 \mathrm{~Hz}, 1 \mathrm{H}), 3.80(\mathrm{~d}, J=13.9 \mathrm{~Hz}, 1 \mathrm{H}), 3.72(\mathrm{dd}, J=$ 7.6, $2.5 \mathrm{~Hz}, 1 \mathrm{H}), 2.91$ (ddd, $J=12.7,8.4,4.3 \mathrm{~Hz}, 1 \mathrm{H}), 2.63-2.56$ (m, 1H), 2.26-2.10 (comp, 2H), 2.03-1.87 (comp, 2H); $\left.{ }^{13} \mathrm{C} \mathrm{NMR} \mathrm{(125} \mathrm{MHz} \mathrm{CDCl}_{3}\right) \delta 147.3,145.2,129.3,123.7,117.6,55.8$, 
$53.4,51.3,29.5,21.9 ; \mathrm{m} / z$ (ESI-MS) $205.2[\mathrm{M}-\mathrm{CN}]^{+}$.

1-(2,4,6-Trimethylbenzyl)pyrrolidine-2-carbonitrile $(\mathbf{4 j})$ : Following the general procedure<smiles>Cc1cc(C)c(CN2CCCC2C#N)c(C)c1</smiles>
compound $4 \mathbf{j}$ was obtained from $L$-proline and mesitaldehyde as a colorless liquid in $95 \%$ yield $\left(\mathrm{R}_{\mathrm{f}}=0.16\right.$ in hexanes $/$ EtOAc $\left.97: 3 \mathrm{v} / \mathrm{v}\right)$; IR (KBr) 2954, 2858, 2221, 1613, 1461, 1374, 1332, 1120, 1046, 851, 665 $\mathrm{cm}^{-1}$; ${ }^{1} \mathrm{H}$ NMR $\left(500 \mathrm{MHz}, \mathrm{CDCl}_{3}\right) 6.84(\mathrm{~s}, 2 \mathrm{H}), 3.85(\mathrm{~d}, J=12.9 \mathrm{~Hz}, 1 \mathrm{H})$, $3.74(\mathrm{~d}, J=12.9 \mathrm{~Hz}, 1 \mathrm{H}), 3.70$ (dd, $J=7.1,3.4 \mathrm{~Hz}, 1 \mathrm{H}), 2.78$ (ddd, $J=$ $13.0,8.5,4.6 \mathrm{~Hz}, 1 \mathrm{H}), 2.66-2.55(\mathrm{~m}, 1 \mathrm{H}), 2.37(\mathrm{~s}, 6 \mathrm{H}), 2.27(\mathrm{~s}, 3 \mathrm{H}), 2.15-$ 2.06 (comp, $2 \mathrm{H}), 1.95-1.85(\mathrm{~m}, 1 \mathrm{H}), 1.85-1.76(\mathrm{~m}, 1 \mathrm{H}) ;{ }^{13} \mathrm{C} \mathrm{NMR}\left(125 \mathrm{MHz}, \mathrm{CDCl}_{3}\right) \delta 137.7$, $136.7,131.2,129.1,118.8,53.5,50.2,49.5,29.7,22.0,20.8,20.0 ; \mathrm{m} / z$ (ESI-MS) 202.0 [M$\mathrm{CN}]^{+}$.

1-(2-Bromobenzyl)pyrrolidine-2-carbonitrile (4k): Following the general procedure<smiles>N#CC1CCCN1Cc1ccccc1Br</smiles>
compound $4 \mathbf{k}$ was obtained from $L$-proline and $o$-bromobenzaldehyde as a colorless liquid in $89 \%$ yield $\left(\mathrm{R}_{\mathrm{f}}=0.19\right.$ in hexanes/EtOAc 93:7 v/v); IR $(\mathrm{KBr})$ $3059,2959,2814,2221,1567,1468,1439,1375,1335,1246,1134,1028,994$, $882,754,660 \mathrm{~cm}^{-1} ;{ }^{1} \mathrm{H}$ NMR $\left(500 \mathrm{MHz}, \mathrm{CDCl}_{3}\right) 7.55$ (app dd, $J=7.9,1.2 \mathrm{~Hz}$, $1 \mathrm{H}), 7.42$ (app dd, $J=7.6,1.6 \mathrm{~Hz}, 1 \mathrm{H}), 7.28$ (app td, $J=7.5,1.2 \mathrm{~Hz}, 1 \mathrm{H}), 7.13$ (app td, $J=7.7,1.7 \mathrm{~Hz}, 1 \mathrm{H}), 3.95(\mathrm{~d}, J=13.8 \mathrm{~Hz}, 1 \mathrm{H}), 3.85(\mathrm{~d}, J=13.8 \mathrm{~Hz}$, 1H), 3.77 (dd, $J=7.5,2.7 \mathrm{~Hz}, 1 \mathrm{H}), 2.92(\mathrm{ddd}, J=12.8,8.4,4.5 \mathrm{~Hz}, 1 \mathrm{H}), 2.66(\mathrm{~m}, 1 \mathrm{H}), 2.40(\mathrm{~s}$, 3H), 2.23-2.09 (comp, 2H), 2.01-1.84 (comp, 2H); ${ }^{13} \mathrm{C} \mathrm{NMR} \mathrm{(125} \mathrm{MHz,} \mathrm{CDCl}_{3}$ ) $\delta 136.8,132.9$, $130.5,128.8,127.3,124.4,118.2,55.9,53.4,51.0,29.6,22.0 ; \mathrm{m} / \mathrm{z}$ (ESI-MS) $239.2[\mathrm{M}-\mathrm{CN}]^{+}$.

1-(Naphthalen-1-ylmethyl)pyrrolidine-2-carbonitrile (4I): Following the general procedure<smiles>N#CC1CCCN1Cc1cccc2ccccc12</smiles>
compound $\mathbf{4 l}$ was obtained from $L$-proline and 1-naphthaldehyde as an offwhite solid in $89 \%$ yield $\left(\mathrm{R}_{\mathrm{f}}=0.27\right.$ in hexanes/EtOAc 95:5 v/v); $)$; mp: $42-$ $44{ }^{\circ} \mathrm{C}$; IR (KBr) 2957, 2817, 2221, 1597, 1509, 1460, 1379, 1331, 1234, $1142,1019,880,779 \mathrm{~cm}^{-1} ;{ }^{1} \mathrm{H}$ NMR $\left(500 \mathrm{MHz}, \mathrm{CDCl}_{3}\right) 8.26$ (app d, $J=8.5$ $\mathrm{Hz}, 1 \mathrm{H}), 7.88(\mathrm{~m}, 1 \mathrm{H}), 7.83$ (app d, $J=8.2 \mathrm{~Hz}, 1 \mathrm{H}), 7.58-7.49$ (comp, 3H), $7.44(\mathrm{~m}, 1 \mathrm{H}), 4.45(\mathrm{~d}, J=12.8 \mathrm{~Hz}, 1 \mathrm{H}), 4.02(\mathrm{~d}, J=12.9 \mathrm{~Hz}, 1 \mathrm{H}), 3.64(\mathrm{~m}$, $1 \mathrm{H}), 3.02$ (ddd, $J=12.7,8.5,4.2,1 \mathrm{H}), 2.72-2.63(\mathrm{~m}, 1 \mathrm{H}), 2.15-2.06$ (comp, $2 \mathrm{H}$ ), 2.02-1.85 (comp, 2H); ${ }^{13} \mathrm{C}$ NMR $\left(125 \mathrm{MHz}, \mathrm{CDCl}_{3}\right) \delta 133.8,133.3,132.1,128.4(2), 128.4(0), 127.2$, $125.9,125.7,125.2,124.3,118.1,54.7,53.2,51.1,29.5,21.8 ; \mathrm{m} / z$ (ESI-MS) $210.1[\mathrm{M}-\mathrm{CN}]^{+}$.

1-(Pyridin-3-ylmethyl)pyrrolidine-2-carbonitrile (4m): Following the general procedure<smiles>N#CC1CCCN1Cc1cccnc1</smiles>
compound $\mathbf{4 m}$ was obtained from $L$-proline and 3-pyridinecarboxaldehyde as a colorless liquid in $88 \%$ yield $\left(\mathrm{R}_{\mathrm{f}}=0.19\right.$ in hexanes/EtOAc $\left.50: 50 \mathrm{v} / \mathrm{v}\right) ; \mathrm{IR}$ (KBr) 2962, 2822, 2222, 1656, 1579, 1479, 1427, 1378, 1330, 1187, 1124, $1029,799,714 \mathrm{~cm}^{-1} ;{ }^{1} \mathrm{H}$ NMR $\left(500 \mathrm{MHz}, \mathrm{CDCl}_{3}\right) 8.54$ (s, $\left.1 \mathrm{H}\right), 8.47$ (app d, $J$ $=4.1 \mathrm{~Hz}, 1 \mathrm{H}), 7.63(\operatorname{app~d}, J=7.9 \mathrm{~Hz}, 1 \mathrm{H}), 7.22(\operatorname{app~dd}, J=7.8,4.8 \mathrm{~Hz}, 1 \mathrm{H})$, $3.85(\mathrm{~d}, J=13.4 \mathrm{~Hz}, 1 \mathrm{H}), 3.65(\mathrm{~d}, J=13.2 \mathrm{~Hz}, 1 \mathrm{H}), 3.67-3.64(\mathrm{~m}, 1 \mathrm{H}), 2.84$ 
(ddd, $J=12.4,8.4,4.4 \mathrm{~Hz}, 1 \mathrm{H}$ ), 2.56-2.49 (comp, 1H), 2.18-2.03 (comp, 2H), 1.96-1.79 (comp, $2 \mathrm{H}) ;{ }^{13} \mathrm{C} \mathrm{NMR}\left(125 \mathrm{MHz}, \mathrm{CDCl}_{3}\right) \delta 149.9,148.7,136.3,133.0,123.3,117.6,53.7,53.1,51.0$, $29.4,21.8 ; \mathrm{m} / z(\mathrm{ESI}-\mathrm{MS}) 188.1[\mathrm{M}+\mathrm{H}]^{+}, 161.2[\mathrm{M}-\mathrm{CN}]^{+}$.

1-(Furan-2-ylmethyl)pyrrolidine-2-carbonitrile (4n): Following the general procedure

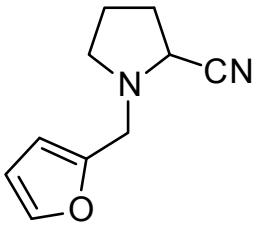
compound 4n was obtained from $L$-proline and furfural as a colorless liquid in $83 \%$ yield $\left(\mathrm{R}_{\mathrm{f}}=0.16\right.$ in hexanes/EtOAc $\left.90: 10 \mathrm{v} / \mathrm{v}\right) ; \mathrm{IR}(\mathrm{KBr}) 2962,2882,2818$, $2222,1601,1505,1445,1372,1335,1224,1149,1014,916,739, \mathrm{~cm}^{-1} ;{ }^{1} \mathrm{H}$ NMR (500 MHz, $\left.\mathrm{CDCl}_{3}\right) 7.40(\mathrm{~m}, 1 \mathrm{H}), 6.33(\mathrm{~m}, 1 \mathrm{H}), 6.29(\mathrm{~d}, J=3.1 \mathrm{~Hz}, 1 \mathrm{H})$, $3.88(\mathrm{~d}, J=13.8 \mathrm{~Hz}, 1 \mathrm{H}), 3.76(\mathrm{~d}, J=13.9 \mathrm{~Hz}, 1 \mathrm{H}), 3.73(\mathrm{dd}, J=7.8,2.8 \mathrm{~Hz}$, $1 \mathrm{H}), 2.96$ (ddd, $J=12.8,8.4,4.4 \mathrm{~Hz}, 1 \mathrm{H}), 2.66-2.59$ (m, 1H), 2.24-2.08 (comp, 2H), 2.02-1.86 (comp, 2H); ${ }^{13} \mathrm{C} \mathrm{NMR}\left(125 \mathrm{MHz}, \mathrm{CDCl}_{3}\right) \delta 151.2,142.8,118.0,110.5,109.1,53.2,51.4,48.7$, $29.8,22.2 ; \mathrm{m} / z(\mathrm{ESI}-\mathrm{MS}) 150.1[\mathrm{M}-\mathrm{CN}]^{+}$.

1-(Thiophen-2-ylmethyl)pyrrolidine-2-carbonitrile (40): Following the general procedure $\square$ compound 40 was obtained from $L$-proline and 2-thiophenecarboxaldehyde as a colorless liquid in 95\% yield $\left(\mathrm{R}_{\mathrm{f}}=0.24\right.$ in hexanes/EtOAc 90:10 v/v); IR $(\mathrm{KBr})$ 2959, 2808, 2222, 1645, 1444, 1377, 1329, 1223, 1117, 951, 851, $696 \mathrm{~cm}^{-1} ;{ }^{1} \mathrm{H}$ NMR $\left(500 \mathrm{MHz}, \mathrm{CDCl}_{3}\right) 7.25(\mathrm{dd}, J=5.1,1.1 \mathrm{~Hz}, 1 \mathrm{H}), 7.00(\mathrm{~m}, 1 \mathrm{H}), 6.94(\mathrm{dd}$, $J=5.1,3.5 \mathrm{~Hz}, 1 \mathrm{H}), 4.07$ (d, $J=13.8 \mathrm{~Hz}, 1 \mathrm{H}), 3.93(\mathrm{~d}, J=13.8 \mathrm{~Hz}, 1 \mathrm{H}), 3.77$ (dd, $J=7.6,2.5 \mathrm{~Hz}, 1 \mathrm{H}), 3.01$ (ddd, $J=12.5,8.3,4.2 \mathrm{~Hz}, 1 \mathrm{H}), 2.64-2.56(\mathrm{~m}, 1 \mathrm{H}), 2.22-2.08$ (comp, 2H), 2.02-1.86 (comp, 2H); ${ }^{13} \mathrm{C} \mathrm{NMR} \mathrm{(125} \mathrm{MHz,} \mathrm{CDCl}_{3}$ ) $\delta 140.6,126.5,126.3,125.4$, $117.7,52.8,51.0,50.7,29.4,21.8 ; \mathrm{m} / z(\mathrm{ESI}-\mathrm{MS}) 166.0[\mathrm{M}-\mathrm{CN}]^{+}$.

1-((1H-Indol-2-yl)methyl)pyrrolidine-2-carbonitrile (4p): Following the general procedure

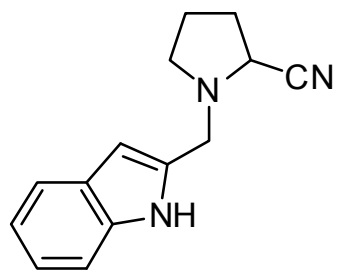
compound 4p was obtained from $L$-proline and indole-2-carboxaldehyde as a colorless liquid in $62 \%$ yield $\left(\mathrm{R}_{\mathrm{f}}=0.25\right.$ in hexanes $\left./ \mathrm{CH}_{2} \mathrm{Cl}_{2} 20: 80 \mathrm{v} / \mathrm{v}\right)$; IR (KBr) 3056, 2961, 2881, 2821, 2224, 1619, 1456, 1421, 1378, 1329, 1289, $1231,1141,1087,995,927,879,791,750 \mathrm{~cm}^{-1} ;{ }^{1} \mathrm{H}$ NMR $(500 \mathrm{MHz}$, $\left.\mathrm{CDCl}_{3}\right) 8.32$ (br s, $\left.1 \mathrm{H}\right), 7.59(\operatorname{app~d}, J=7.8 \mathrm{~Hz}, 1 \mathrm{H}), 7.34$ (app dd, $J=8.1$, $0.7 \mathrm{~Hz}, 1 \mathrm{H}), 7.19(\mathrm{~m}, 1 \mathrm{H}), 7.11(\mathrm{~m}, 1 \mathrm{H}), 6.47$ (s, 1H), 4.08 (d, $J=13.6 \mathrm{~Hz}$, $1 \mathrm{H}), 3.86(\mathrm{~d}, J=13.7 \mathrm{~Hz}, 1 \mathrm{H}), 3.72(\mathrm{dd}, J=7.2,3.0 \mathrm{~Hz}, 1 \mathrm{H}), 2.95$ (ddd, $J=12.7,8.4,4.3 \mathrm{~Hz}$, $1 \mathrm{H}), 2.69-2.61(\mathrm{~m}, 1 \mathrm{H}), 2.23-2.11$ (comp, $2 \mathrm{H}), 2.05-1.88$ (comp, $2 \mathrm{H}) ;{ }^{13} \mathrm{C}$ NMR $(125 \mathrm{MHz}$, $\left.\mathrm{CDCl}_{3}\right) \delta 136.1,134.7,128.1,121.8,120.4,119.7,117.8,110.7,101.8,53.2,51.3,49.5,29.4$, $21.9 ; \mathrm{m} / z$ (ESI-MS) $199.0[\mathrm{M}-\mathrm{CN}]^{+}$.

Ethyl 2-(2-cyanopyrrolidin-1-yl)acetate (4q): Following the general procedure compound 4q<smiles>CCOC(=O)CN1CCCC1C#N</smiles>
was obtained from $L$-proline and ethyl glyoxalate solution ( $\sim 50 \%$ in toluene) as a colorless liquid in $97 \%$ yield $\left(\mathrm{R}_{\mathrm{f}}=0.26\right.$ in hexanes $/$ EtOAc $\left.80: 20 \mathrm{v} / \mathrm{v}\right)$; IR (KBr) 2982, 2822, 2220, 1743, 1464, 1428, 1384, 1200, 1160, 1028, $863 \mathrm{~cm}^{-1}$; ${ }^{1} \mathrm{H} \mathrm{NMR}\left(500 \mathrm{MHz}, \mathrm{CDCl}_{3}\right) 4.13(\mathrm{t}, J=4.1 \mathrm{~Hz}, 2 \mathrm{H}), 4.08(\mathrm{dd}, J=7.8,4.1 \mathrm{~Hz}$, 1H), 3.51-3.40 (comp, 2H), 3.00 (ddd, $J=13.0,8.8,5.1 \mathrm{~Hz}, 1 \mathrm{H}), 2.66-2.58$ (m, 
1H), 2.26-2.16 (m, 1H), 2.12-2.14 (m, 1H), 1.95-1.84 (comp, $2 \mathrm{H}), 1.22(\mathrm{t}, J=7.2 \mathrm{~Hz}, 3 \mathrm{H}) ;{ }^{13} \mathrm{C}$ $\operatorname{NMR}\left(125 \mathrm{MHz}, \mathrm{CDCl}_{3}\right) \delta 169.7,118.0,60.7,52.8,52.4,51.3,29.8,22.1,13.9 ; \mathrm{m} / z(\mathrm{ESI}-\mathrm{MS})$ $156.1[\mathrm{M}-\mathrm{CN}]^{+}$.

1-(3-Phenylpropyl)pyrrolidine-2-carbonitrile (4r): Following the general procedure compound

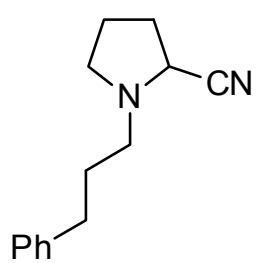
4r was obtained from $L$-proline and hydrocinnamaldehyde as colorless liquid in $72 \%$ yield $\left(\mathrm{R}_{\mathrm{f}}=0.16\right.$ in hexanes/EtOAc 90:10 v/v); IR $(\mathrm{KBr}) 3026,2942,2813$, 2220, 1602, 1496, 1454, 1386, 1318, 1182, 1145, 1123, 1079, 1030, 966, 882, 747, $700 \mathrm{~cm}^{-1}$; ${ }^{1} \mathrm{H}$ NMR (500 MHz, $\mathrm{CDCl}_{3}$ ) 7.33-7.27 (comp, 2H), 7.23-7.18 (comp, 3H), 3.76 (dd, $J=7.6,2.8 \mathrm{~Hz}, 1 \mathrm{H}$ ), 2.89 (ddd, $J=12.9,8.4,4.6 \mathrm{~Hz}, 1 \mathrm{H}$ ), $2.73(\mathrm{~m}, 1 \mathrm{H}), 2.69(\mathrm{t}, J=7.6 \mathrm{~Hz}, 2 \mathrm{H}), 2.64-2.52$ (comp, 2H), 2.23-2.08 (comp, 2H), 2.01-1.82 (comp, 4H); ${ }^{13} \mathrm{C}$ NMR (125 MHz, $\left.\mathrm{CDCl}_{3}\right) \delta 141.7,128.3,128.2,125.8,118.1$, $53.6,51.8,51.0,33.3,30.0,29.5,21.8 ; \mathrm{m} / z$ (ESI-MS) $188.3[\mathrm{M}-\mathrm{CN}]^{+}$.

1-Phenethylpyrrolidine-2-carbonitrile (4s): Following the general procedure compound 4s was

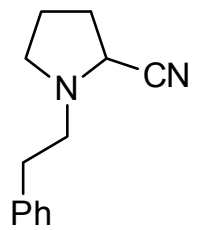
obtained from $L$-proline and phenylacetaldehyde as a colorless liquid in $91 \%$ yield $\left(\mathrm{R}_{\mathrm{f}}=0.24\right.$ in hexanes/EtOAc 85:15 v/v); IR (KBr) 3027, 2949, 2815, 2220, 1603, $1497,1454,1383,1342,1181,1146,1122,1079,1030,884,751,700 \mathrm{~cm}^{-1} ;{ }^{1} \mathrm{H}$ NMR (500 MHz, $\mathrm{CDCl}_{3}$ ) 7.33-7.27 (comp, 2H), 7.25-7.18 (comp, 3H), 3.84 (dd, $J$ $=7.4,2.6 \mathrm{~Hz}, 1 \mathrm{H}), 3.01-2.91$ (comp, 2H), 2.88-2.78 (comp, 3H), 2.63-2.57 (m, $1 \mathrm{H}), 2.23-2.09$ (comp, 2H), 2.01-1.85 (comp, 2H); $\left.{ }^{13} \mathrm{C} \mathrm{NMR} \mathrm{(125} \mathrm{MHz,} \mathrm{CDCl}_{3}\right) \delta 139.5,128.6$, $128.4,126.2,118.0,54.2,53.7,51.3,35.1,29.6,21.9 ; \mathrm{m} / z(\mathrm{ESI}-\mathrm{MS}) 174.2[\mathrm{M}-\mathrm{CN}]^{+}$.

1-(Cyclohexylmethyl)pyrrolidine-2-carbonitrile (4t): Following the general procedure<smiles>N#CC1CCCN1CC1CCCCC1</smiles>
compound $\mathbf{4 t}$ was obtained from $L$-proline and cyclohexanecarboxaldehyde as a colorless liquid in 97\% yield as a mixture of regioisomers; $\mathrm{rr}=2.2: 1\left(\mathrm{R}_{\mathrm{f}}=0.23\right.$ in hexanes/EtOAc 95:5 v/v); Characterization data of the major regioisomer: IR (KBr) 2923, 2851, 2810, 2221, 1449, 1341, 1244, 1189, 1147, 1114, 1082, $879 \mathrm{~cm}^{-1}$; ${ }^{1} \mathrm{H}$ NMR $\left(500 \mathrm{MHz}, \mathrm{CDCl}_{3}\right) 3.72(\mathrm{dd}, J=7.5,2.4 \mathrm{~Hz}, 1 \mathrm{H}), 2.83(\mathrm{~m}$, 1H), 2.54-2.42 (comp, 2H), 2.40-2.33 (m, 1H), 2.19-2.04 (comp, 2H), 1.961.81 (comp, 2H), 1.80-1.73 (comp, 2H), 1.73-1.60 (comp, 2H), 1.48-1.37 (m, 1H), 1.31-1.09 (comp, 4H), 0.94-0.74 (comp, 2H); $\left.{ }^{13} \mathrm{C} \mathrm{NMR} \mathrm{(125} \mathrm{MHz,} \mathrm{CDCl}_{3}\right) \delta 118.3,59.4,54.1,51.4,36.5$, 31.6, 31.5, 29.6, 26.7, 25.9(2), 25.9(1), 21.9; $\mathrm{m} / \mathrm{z}$ (ESI-MS) $166.2[\mathrm{M}-\mathrm{CN}]^{+}$.

1-Isopentylpyrrolidine-2-carbonitrile (4u): Following the general procedure compound $\mathbf{4 u}$ was

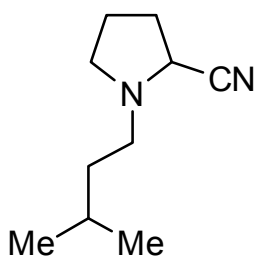
obtained from $L$-proline and isovaleraldehyde as a colorless liquid in $82 \%$ yield as a mixture of regioisomers; $\mathrm{rr}=4.6: 1\left(\mathrm{R}_{\mathrm{f}}=0.25\right.$ in hexanes/EtOAc 90:10 v/v); Characterization data of the major regioisomer: IR (KBr) 2956, 2870, 2813, 2220, 1468, 1385, 1367, 1152, 1125, 1097, $885 \mathrm{~cm}^{-1}$; ${ }^{1} \mathrm{H}$ NMR (500 MHz, $\left.\mathrm{CDCl}_{3}\right) 3.75(\mathrm{dd}, J=7.3,2.7 \mathrm{~Hz}, 1 \mathrm{H}), 2.87(\mathrm{ddd}, J=12.8,8.3,4.5 \mathrm{~Hz}, 1 \mathrm{H})$, 2.72-2.62 (m. 1H), 2.57-2.46 (comp, 2H), 2.20-2.05 (comp, 2H), 1.97-1.80 (comp, 2H), 1.62 (app sept, $J=6.7 \mathrm{~Hz}, 1 \mathrm{H}), 1.41-1.33$ (comp, 2H), $0.90(\mathrm{~d}, J=6.7 \mathrm{~Hz}, 6 \mathrm{H}) ;{ }^{13} \mathrm{C}$ 
$\operatorname{NMR}\left(125 \mathrm{MHz}, \mathrm{CDCl}_{3}\right) \delta 118.1,53.7,51.2,50.8,37.3,29.5,26.2,22.7,22.5,21.8 ; \mathrm{m} / z(\mathrm{ESI}-$ MS) $140.1[\mathrm{M}-\mathrm{CN}]^{+}$.

1-Benzhydrylpyrrolidine-2-carbonitrile (4v): Following the general procedure compound 4v $\square$ was obtained from $L$-proline and benzophenone as a white solid in $57 \%$ yield $\left(\mathrm{R}_{\mathrm{f}}=\right.$ N 0.22 in hexanes/EtOAc 97:3 v/v); mp: 108-111 ${ }^{\circ} \mathrm{C}$; IR (KBr) 3061, 3028, 2958, Ph $2821,2222,1598,1491,1453,1306,1186,1130,1076,1028,927,887,748,706$, 7.19 (comp, 2H), $4.61(\mathrm{~s}, 1 \mathrm{H}), 3.78(\operatorname{app~d}, J=7.3 \mathrm{~Hz}, 1 \mathrm{H}), 3.02-2.94(\mathrm{~m}, 1 \mathrm{H}), 2.44-2.36(\mathrm{~m}$, $1 \mathrm{H}), 2.25-2.14(\mathrm{~m}, 1 \mathrm{H}), 2.13-2.03(\mathrm{~m}, 1 \mathrm{H}), 2.01-1.87$ (comp, $2 \mathrm{H}) ;{ }^{13} \mathrm{C} \mathrm{NMR}\left(125 \mathrm{MHz}, \mathrm{CDCl}_{3}\right)$ $\delta 142.5,142.0,128.8,128.6,127.6,127.5,127.3(1), 127.3(0), 117.7,71.8,53.1,49.9,29.4,21.8$; $\mathrm{m} / \mathrm{z}(\mathrm{ESI}-\mathrm{MS}) 136.0[\mathrm{M}-\mathrm{CN}]^{+}$.

2-Benzyl-1,2,3,4-tetrahydroisoquinoline-3-carbonitrile (7): Following the general procedure compound 7 was obtained from (S)-(-)-1,2,3,4-tetrahydro-31 isoquinolinecarboxylic acid and benzaldehyde as an off-white solid in $91 \%$ yield $\left(\mathrm{R}_{\mathrm{f}}=0.21\right.$ in hexanes/EtOAc 93:7 v/v); mp: 110-112 ${ }^{\circ} \mathrm{C} ; \mathrm{IR}(\mathrm{KBr}) 2818,2222$,<smiles>CN(CC=[W])Cc1ccccc1</smiles>
$1644,1496,1455,1357,1315,1145,1091,1074,1028,989,741,701 \mathrm{~cm}^{-1} ;{ }^{1} \mathrm{H}$ NMR (500 MHz, $\left.\mathrm{CDCl}_{3}\right)$ 7.42-7.30 (comp, 5H), 7.21-7.15 (comp, 2H), 7.13$7.10(\mathrm{~m}, 1 \mathrm{H}), 7.06(\mathrm{~m}, 1 \mathrm{H}), 4.03(\mathrm{~d}, J=6.3 \mathrm{~Hz}, 1 \mathrm{H}), 3.98(\mathrm{~d}, J=15.6 \mathrm{~Hz}, 1 \mathrm{H})$, $3.92(\mathrm{~d}, J=13.2 \mathrm{~Hz}, 1 \mathrm{H}), 3.78(\mathrm{~d}, J=15.5 \mathrm{~Hz}, 1 \mathrm{H}), 3.70(\mathrm{~d}, J=13.2 \mathrm{~Hz}, 1 \mathrm{H}), 3.31$ (dd, $J=16.3$, $6.1 \mathrm{~Hz}, 1 \mathrm{H}), 2.98(\mathrm{~d}, J=16.5 \mathrm{~Hz}, 1 \mathrm{H}) ;{ }^{13} \mathrm{C} \mathrm{NMR}\left(125 \mathrm{MHz}, \mathrm{CDCl}_{3}\right) \delta 136.4,132.8,129.8$, 129.0, 128.7(2), 128.7(0), 127.9, 126.7, 126.6, 126.5, 116.3, 60.2, 51.6, 49.3, 32.6; m/z (ESI-MS) $222.2[\mathrm{M}-\mathrm{CN}]^{+}$.

\section{References:}

[1] (a) Ficini, J.; Normant, H. Bull. Soc. Chim. Fr., 1957, 1454; (b) Sakai, H.; Ito, K.; Sekiya, M. Chem. Pharm. Bull., 1973, 21, 2257.

[2] Ho, B.; Castagnoli, Jr, N. J. Med. Chem., 1980, 23, 133.

[3] (a) Gall, E. L.; Hurvois, J.-P.; Sinbandhit, S. Eur. J. Org. Chem., 1999, 2645; (b) Bahde, R. J.; Rychnovsky, S. D. Org. Lett., 2008, 10, 4017.

[4] Harcourt, D. N.; Waigh, R. D. J. Chem. Soc. C, 1971, 967.

[5] Mojtahedi, M. M.; Abaee, M. S.; Alishiri, T. Tetrahedron Lett., 2009, 50, 2322.

[6] Yoshikai, N.; Mieczkowski, A.; Matsumoto, A.; Ilies, L.; Nakamura, E. J. Am. Chem. Soc., 2010, 132, 5568.

[7] Xiao, K.-J.; Wang, Y.; Ye, K.-Y.; Huang, P.-Q. Chem.-Eur. J., 2010, 16, 12792. 
${ }^{1} \mathrm{H}$ NMR of $\mathbf{4 b}$ in $\mathrm{CDCl}_{3}$
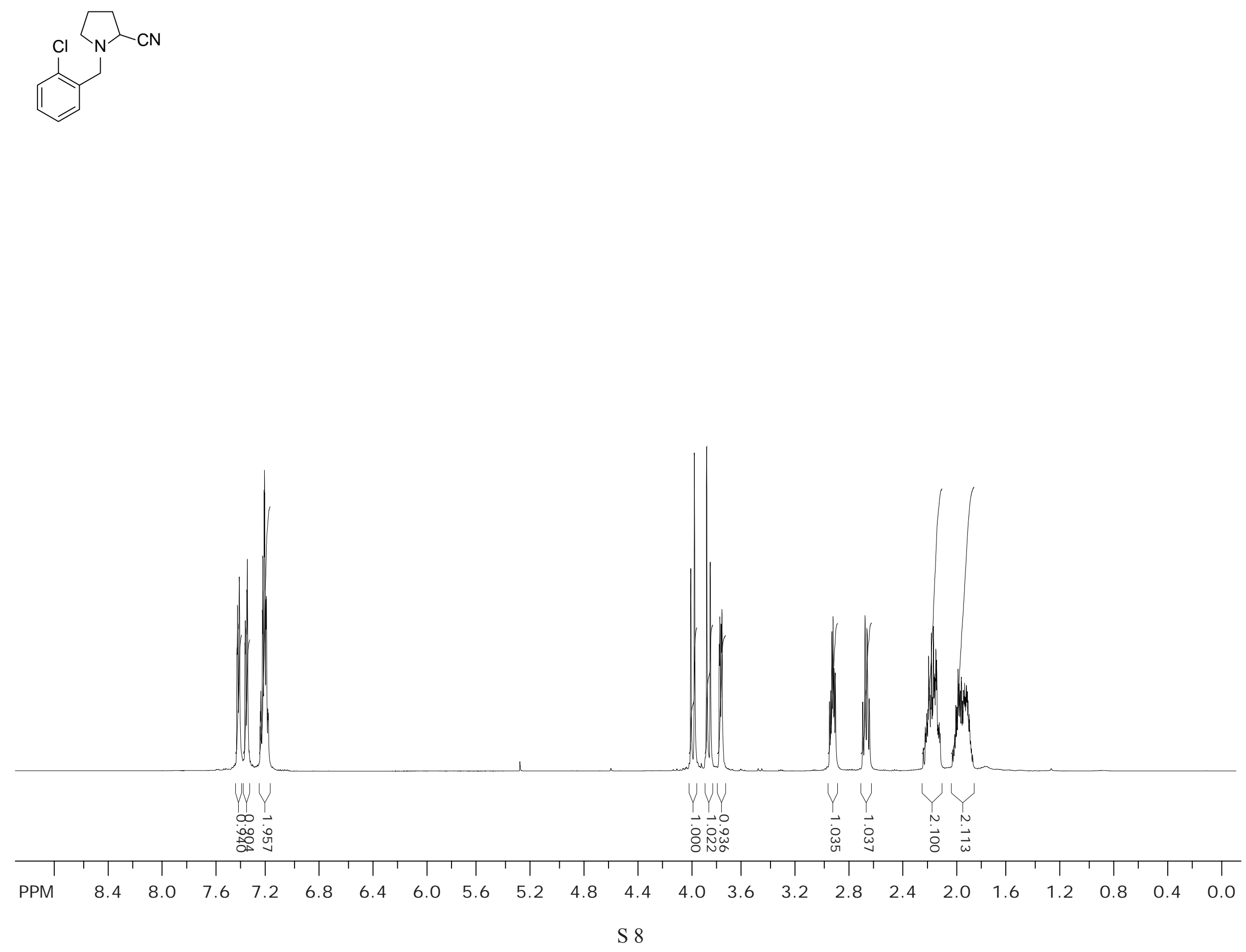
${ }^{13} \mathrm{C} \mathrm{NMR}$ of $\mathbf{4 b}$ in $\mathrm{CDCl}_{3}$

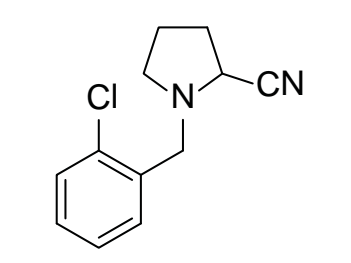

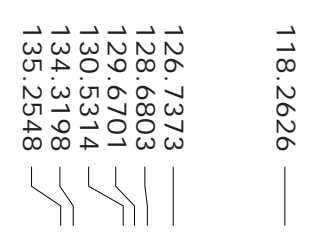

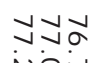

जio

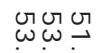

vin

NNG

Y)

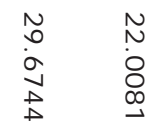
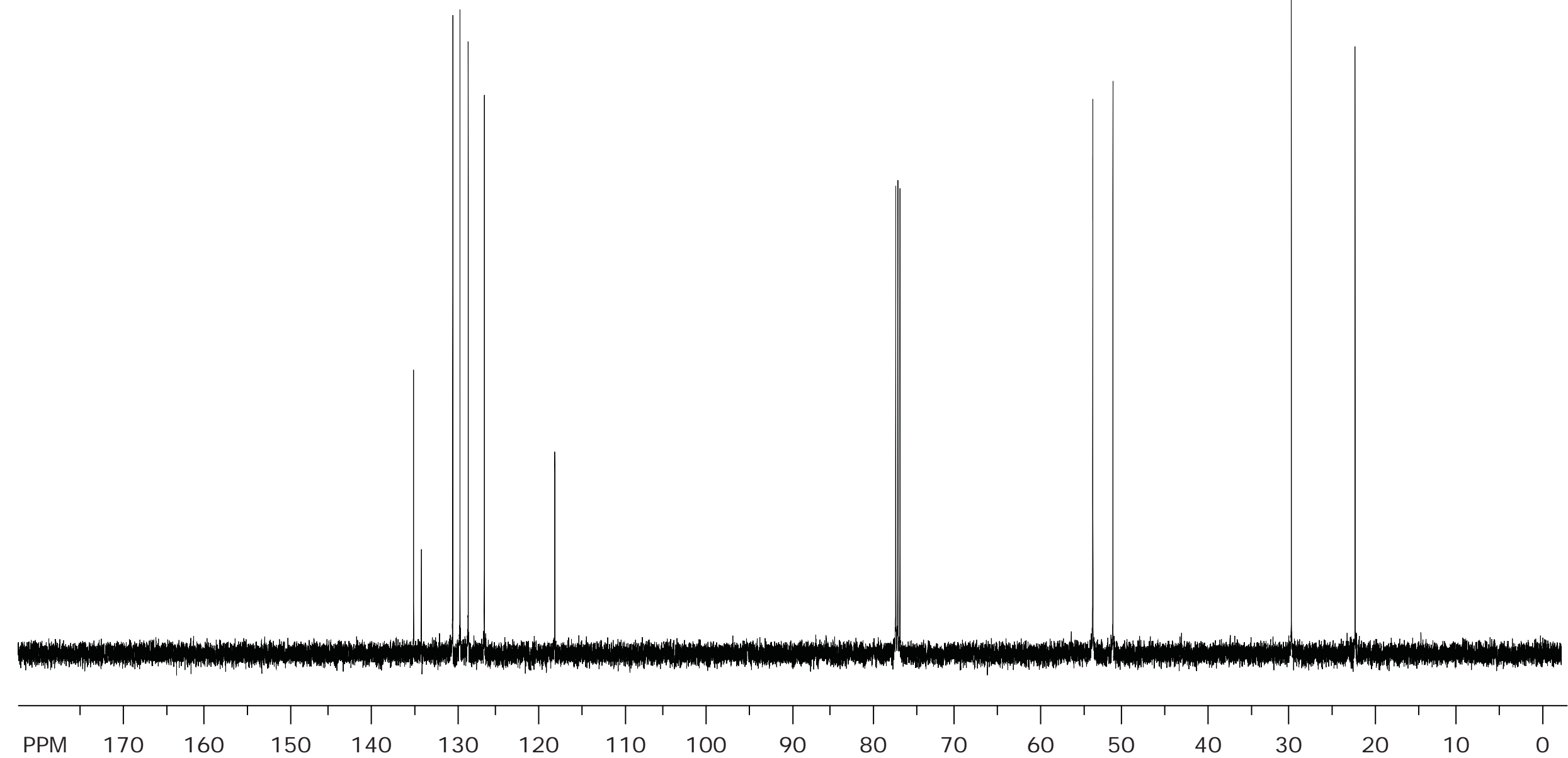

S 9 
${ }^{1} \mathrm{H} \mathrm{NMR}$ of $4 \mathrm{C}$ in $\mathrm{CDCl}_{3}$
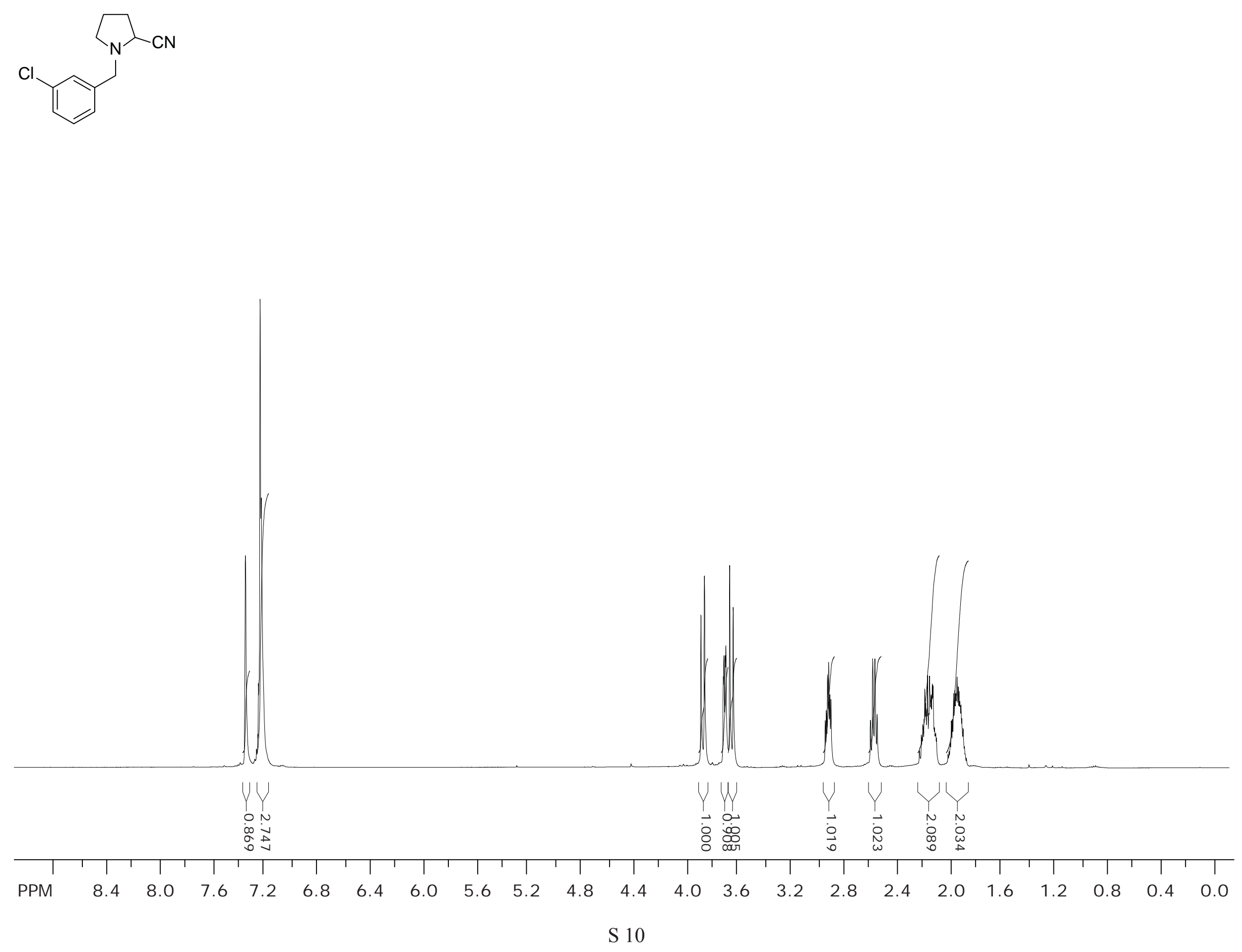
${ }^{13} \mathrm{C}$ NMR of $4 \mathrm{c}$ in $\mathrm{CDCl}_{3}$
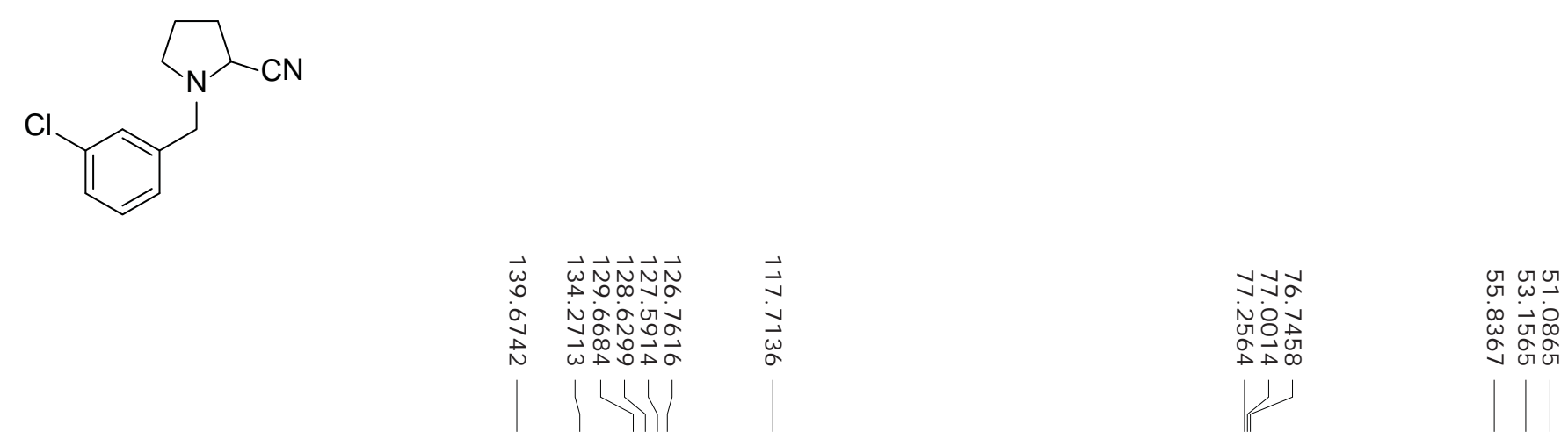

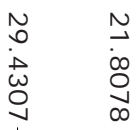
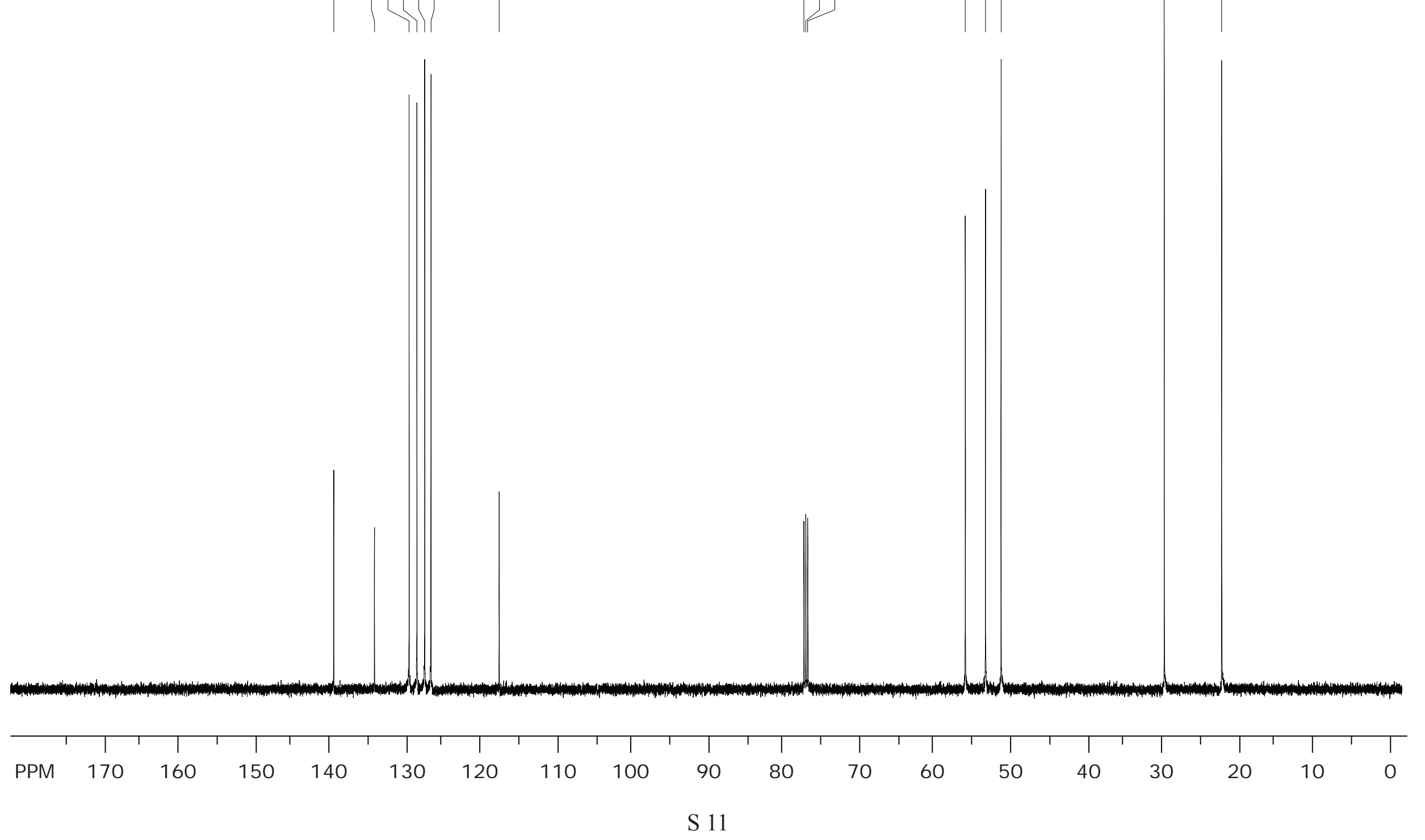


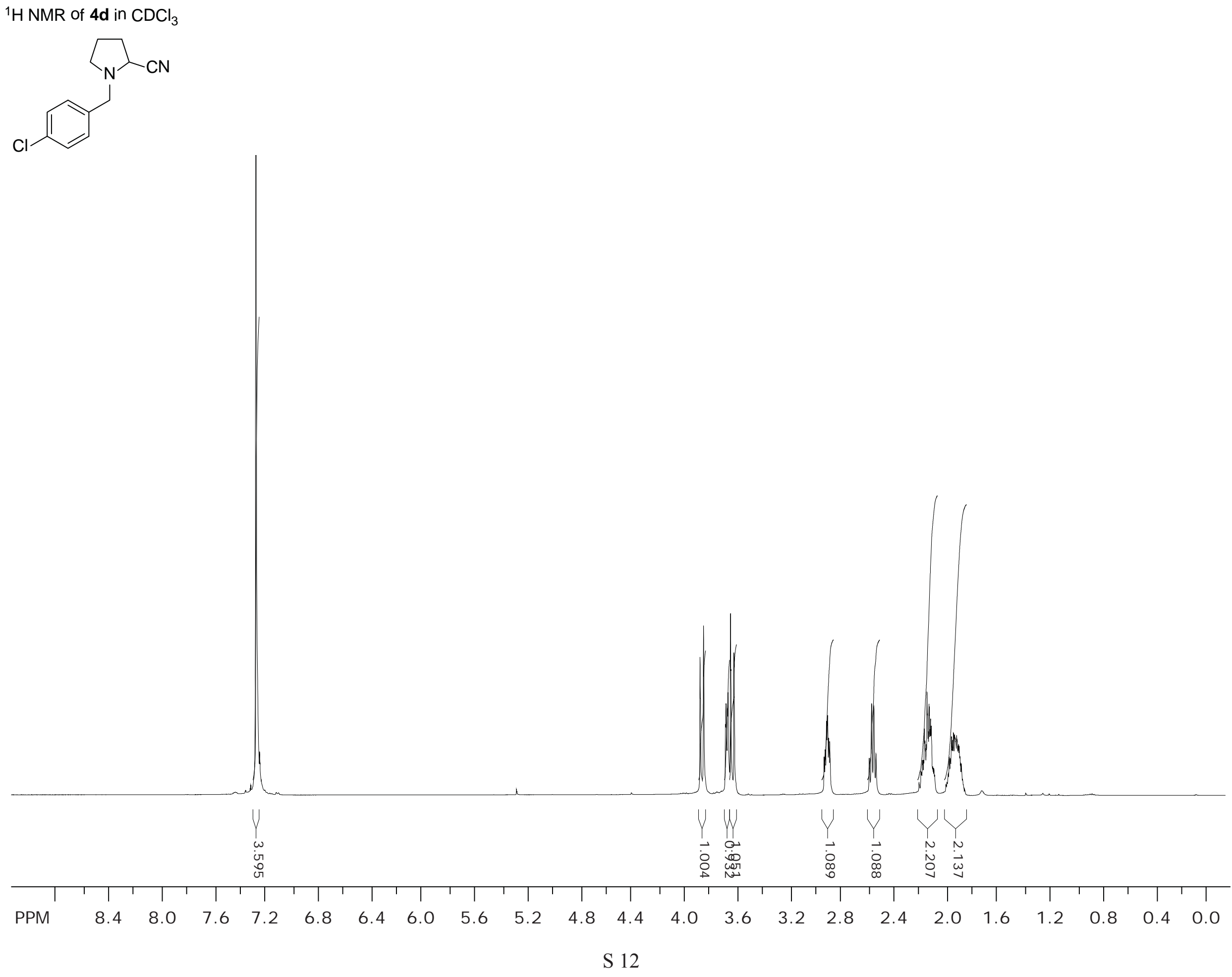


${ }^{13} \mathrm{C}$ NMR of $\mathbf{4 d}$ in $\mathrm{CDCl}_{3}$
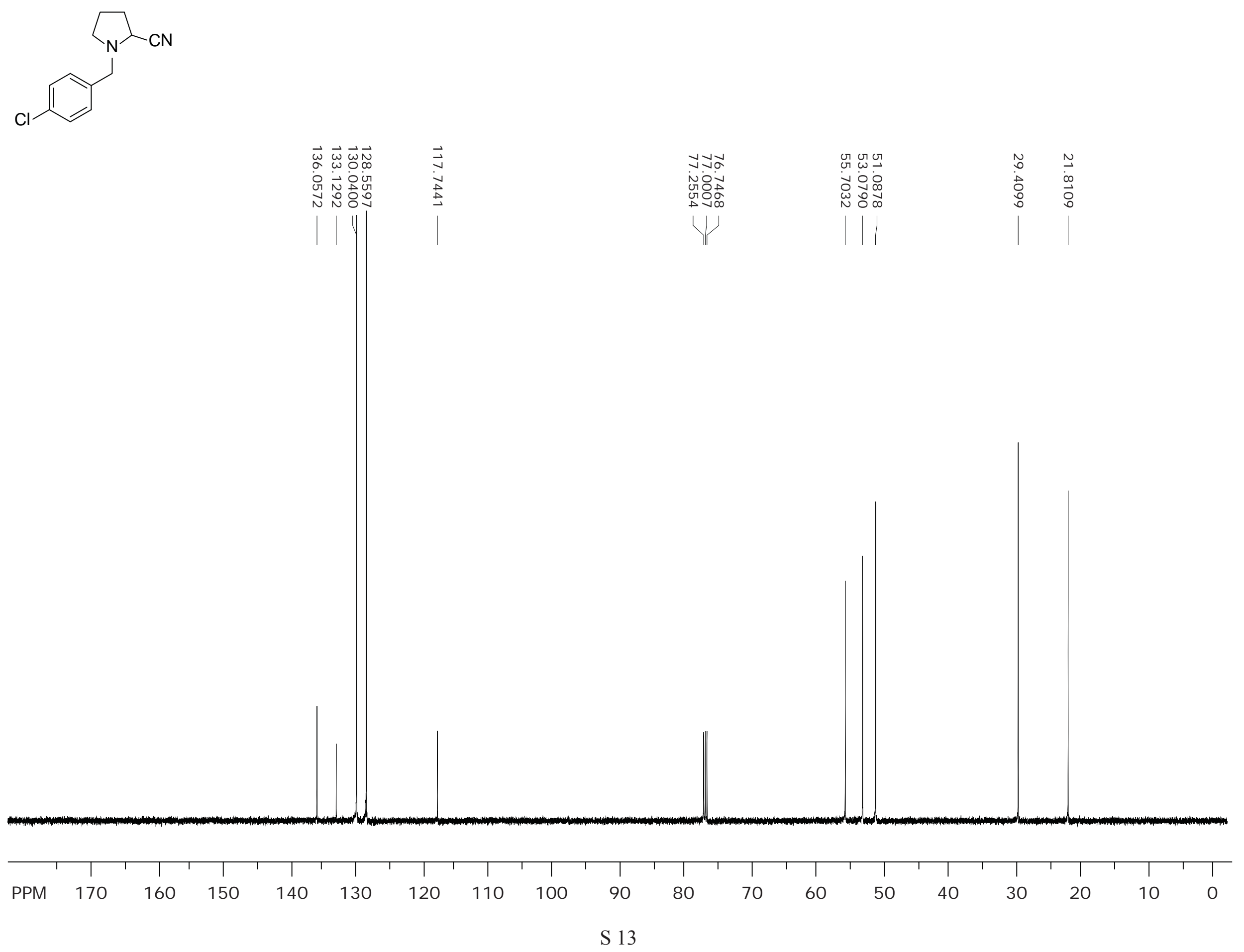
${ }^{1} \mathrm{H} \mathrm{NMR}$ of $4 \mathbf{e}$ in $\mathrm{CDCl}_{3}$
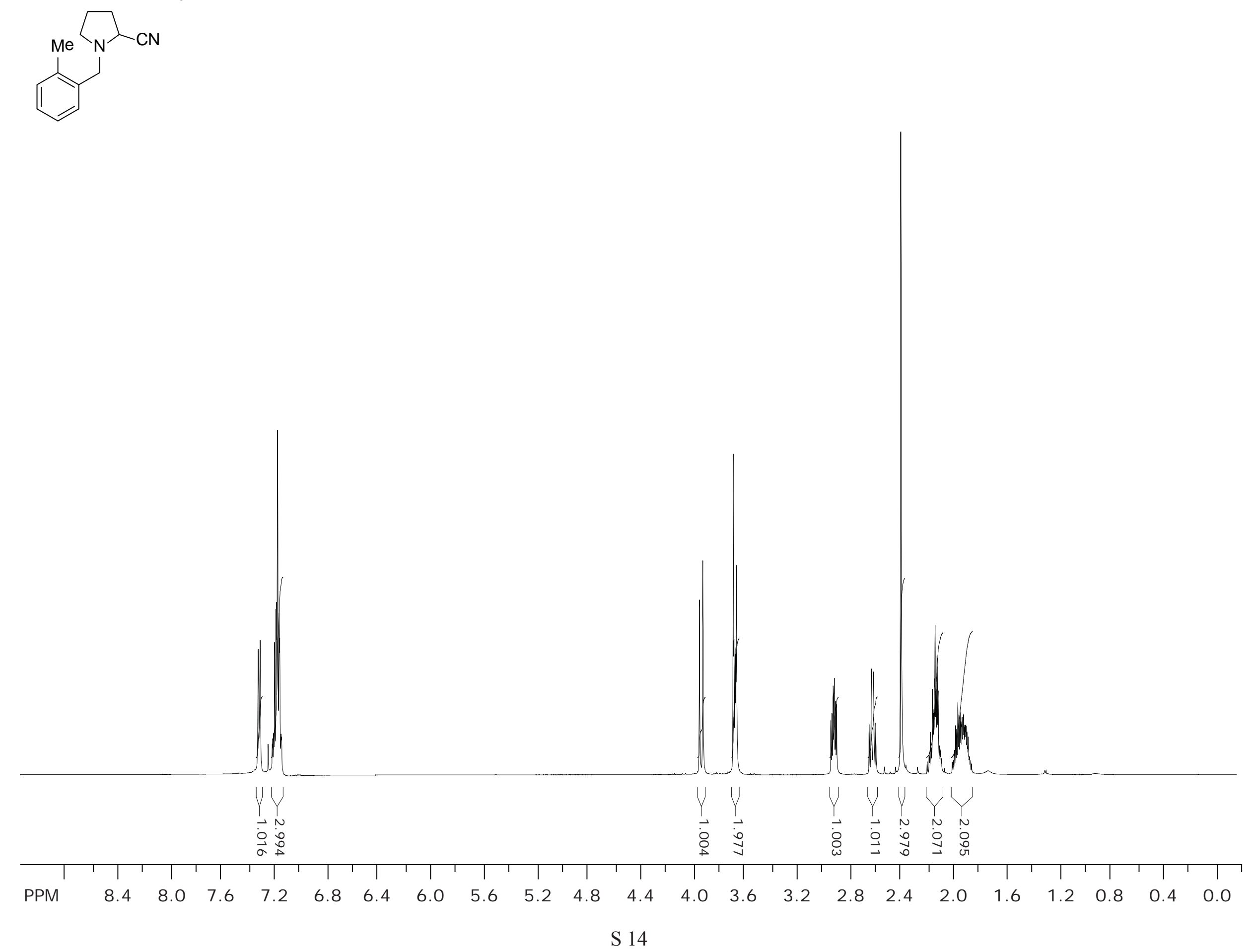
${ }^{13} \mathrm{C} \mathrm{NMR}$ of $4 \mathbf{e}$ in $\mathrm{CDCl}_{3}$
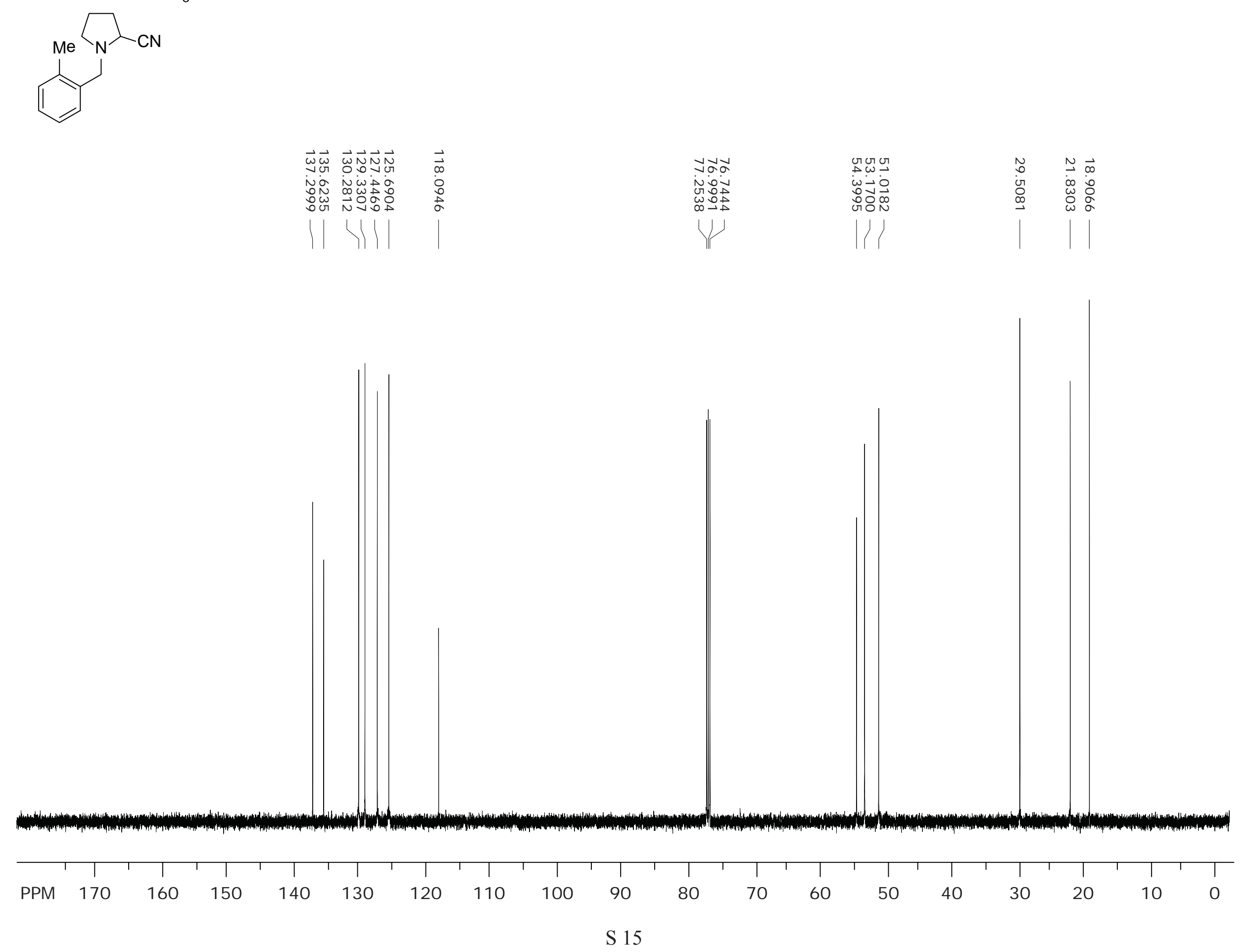


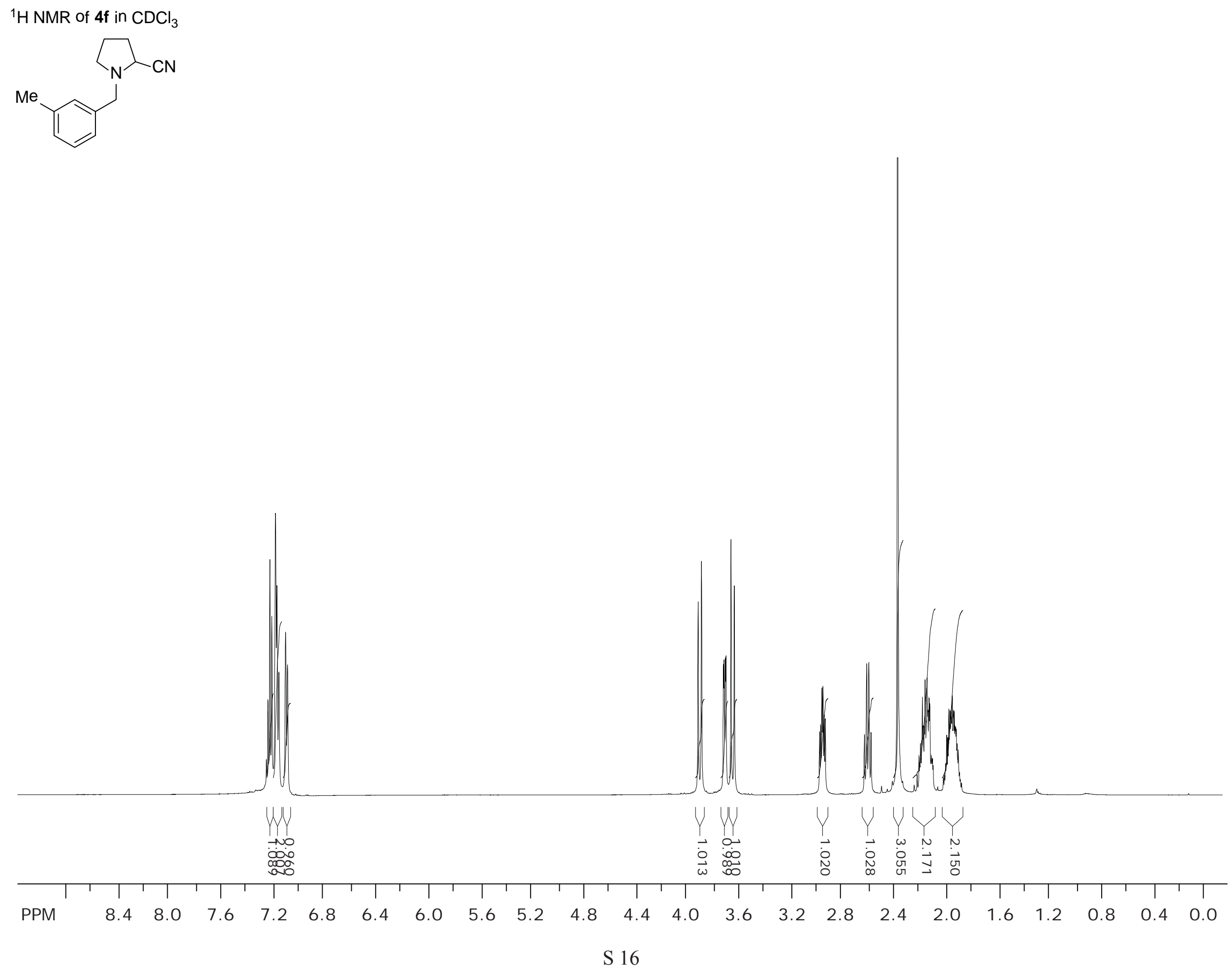


${ }^{13} \mathrm{C}$ NMR of $\mathbf{4 f}$ in $\mathrm{CDCl}_{3}$
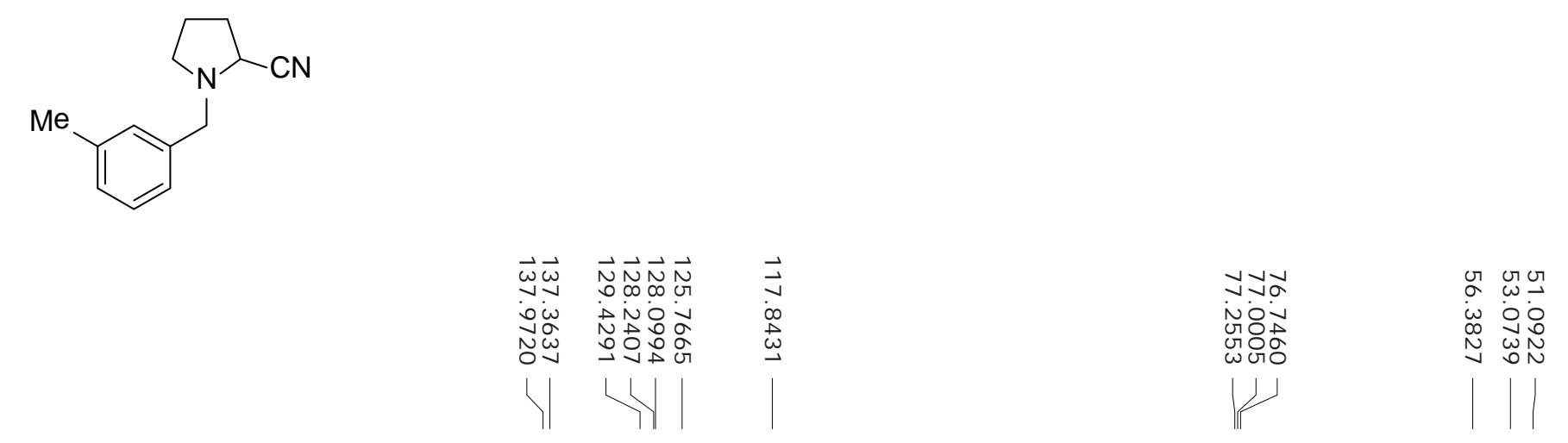

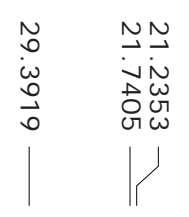

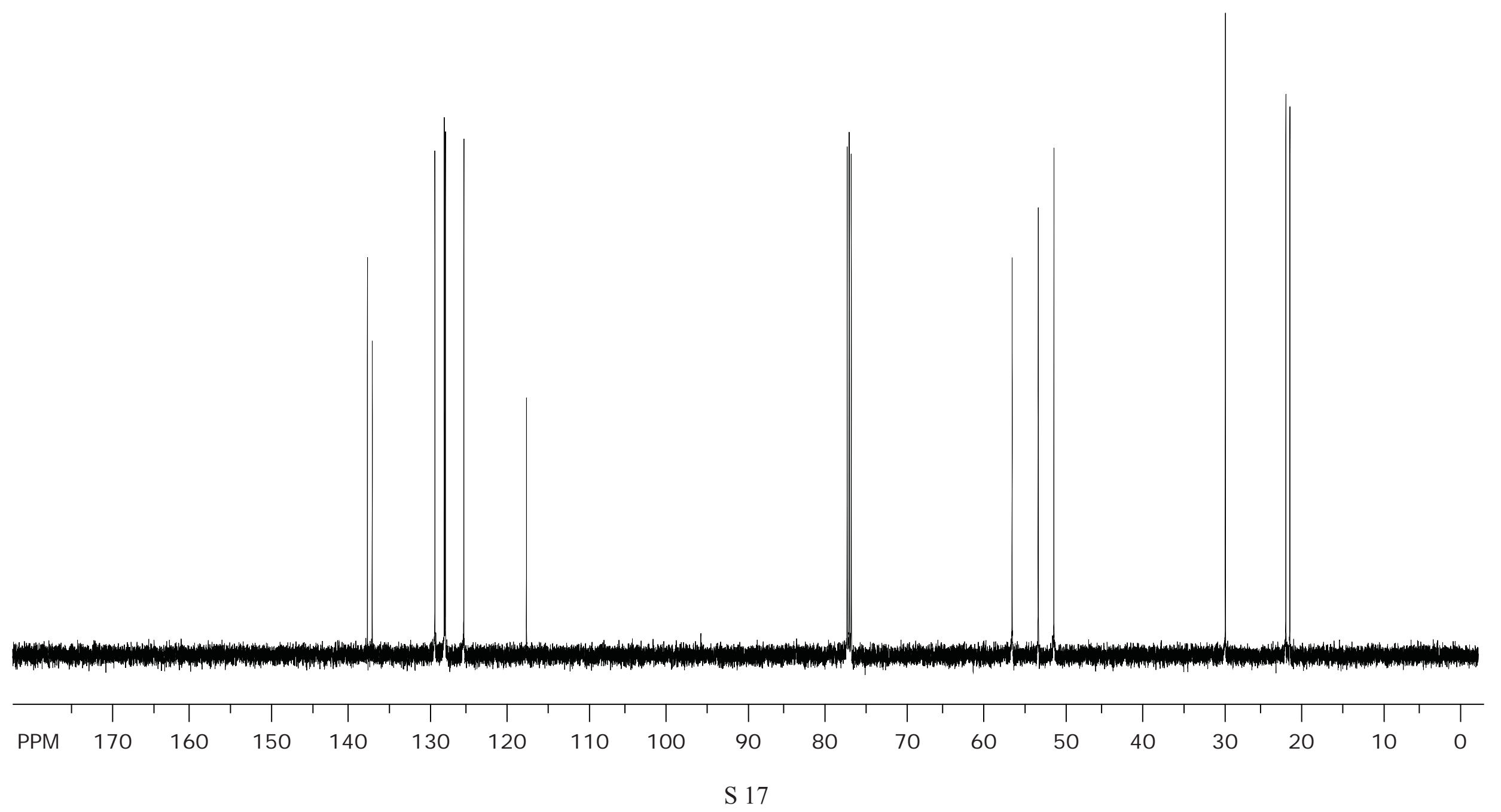


${ }^{1} \mathrm{H}$ NMR of $\mathbf{4} \mathbf{g}$ in $\mathrm{CDCl}_{3}$
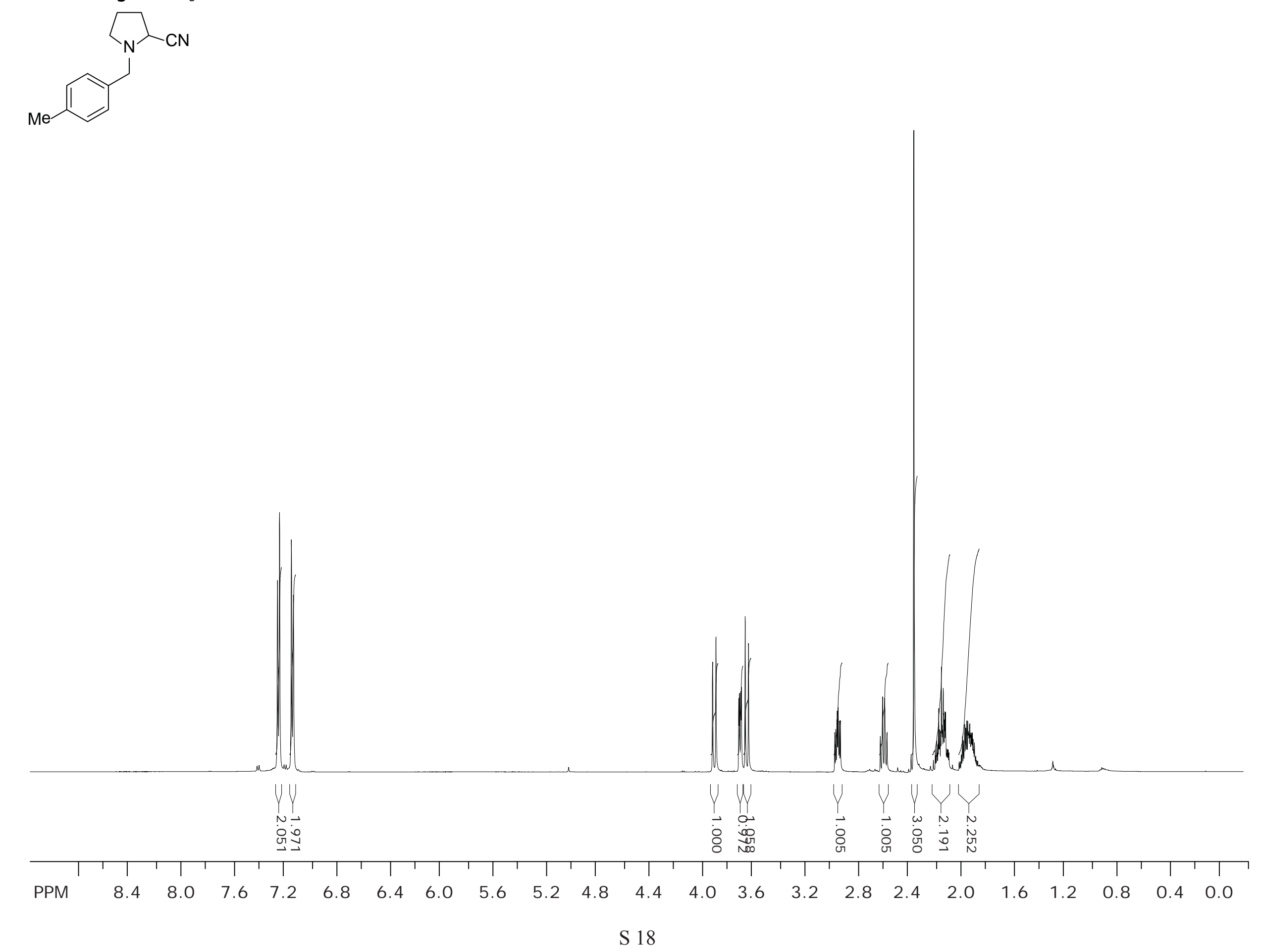
${ }^{13} \mathrm{C} \mathrm{NMR}$ of $\mathbf{4} \mathbf{g}$ in $\mathrm{CDCl}_{3}$
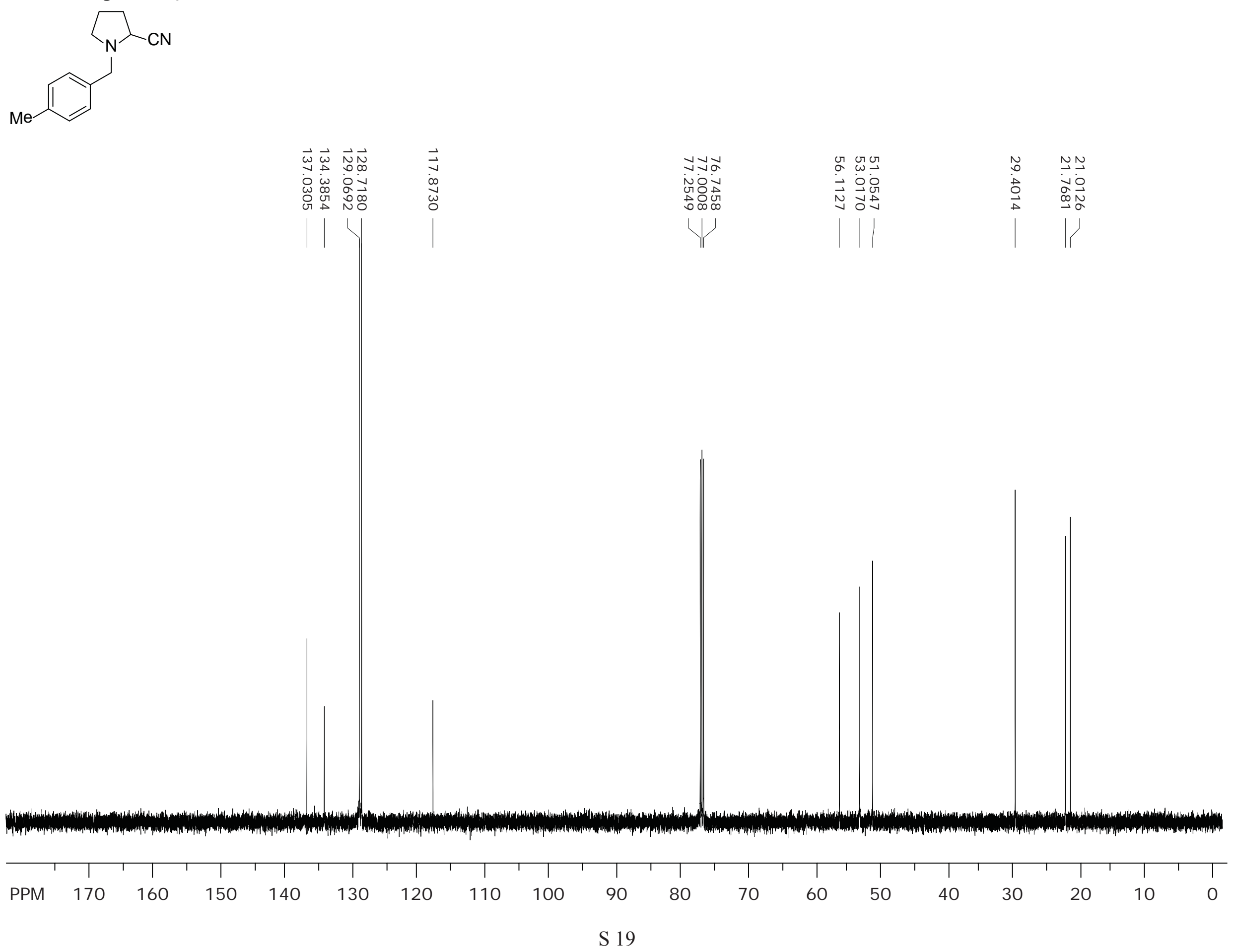
${ }^{1} \mathrm{H}$ NMR of $4 \mathrm{~h}$ in $\mathrm{CDCl}_{3}$
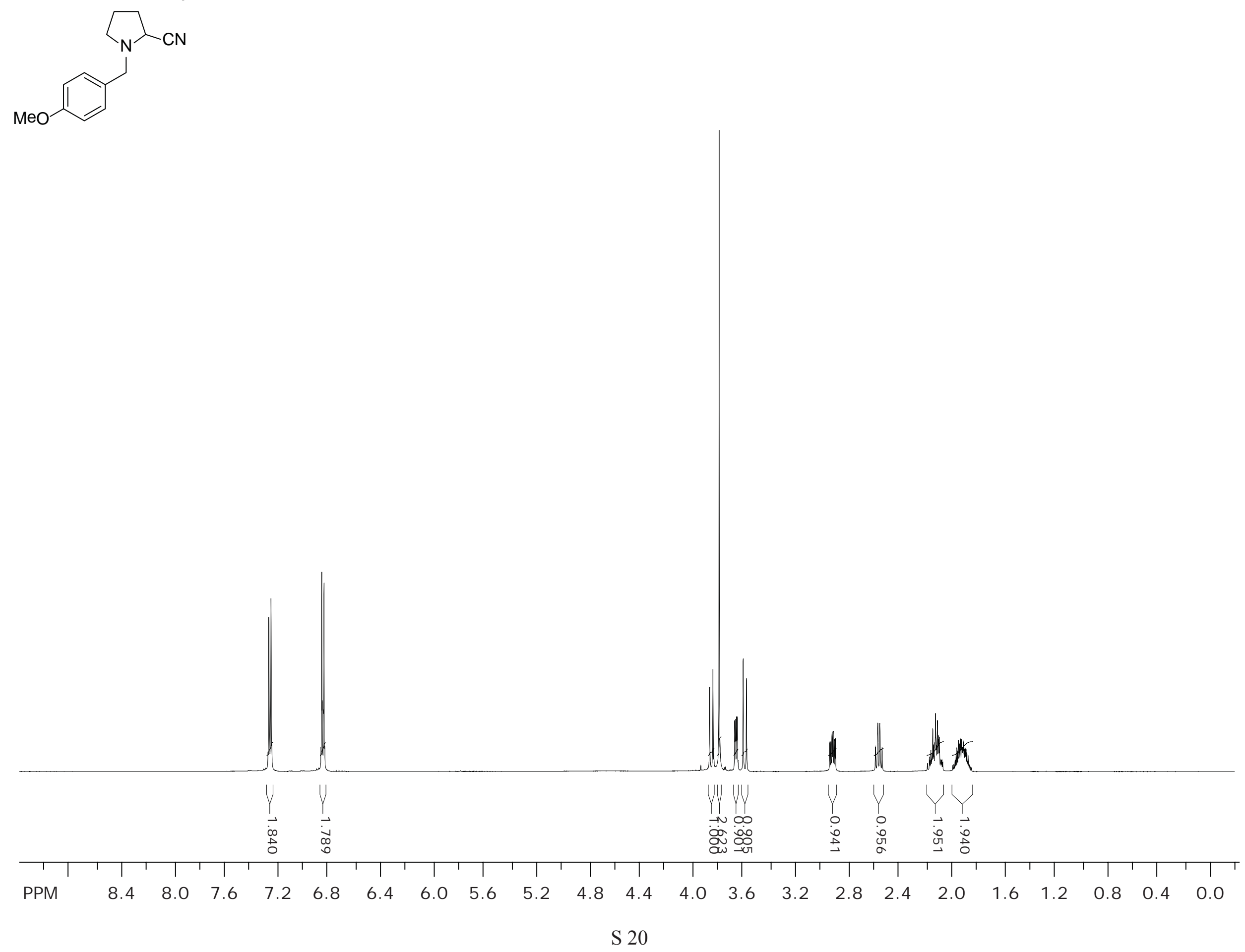
${ }^{13} \mathrm{C} \mathrm{NMR}$ of $4 \mathrm{~h}$ in $\mathrm{CDCl}_{3}$
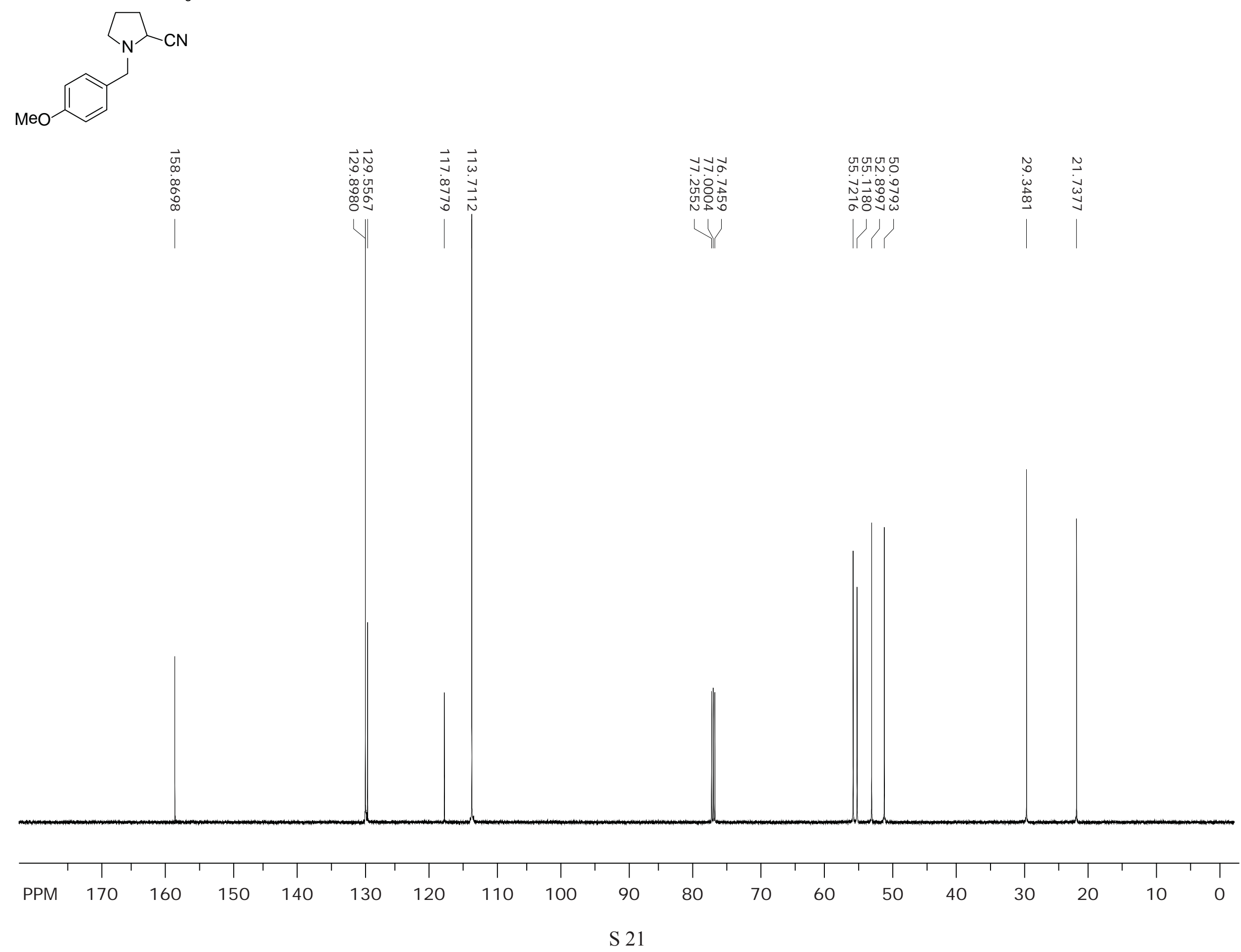
${ }^{1} \mathrm{H}$ NMR of $4 \mathbf{i}$ in $\mathrm{CDCl}_{3}$
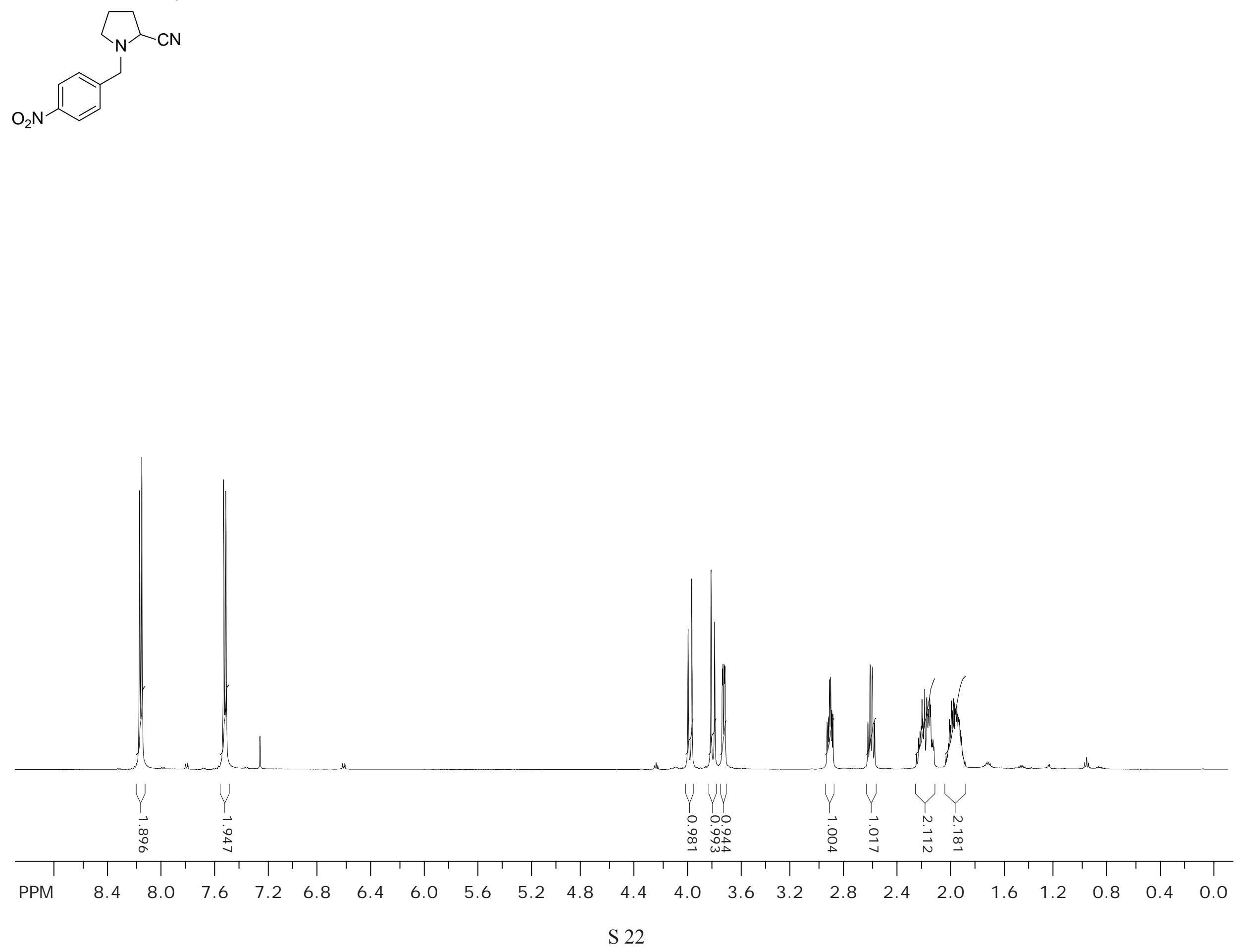
${ }^{13} \mathrm{C}$ NMR of $4 \mathbf{i}$ in $\mathrm{CDCl}_{3}$
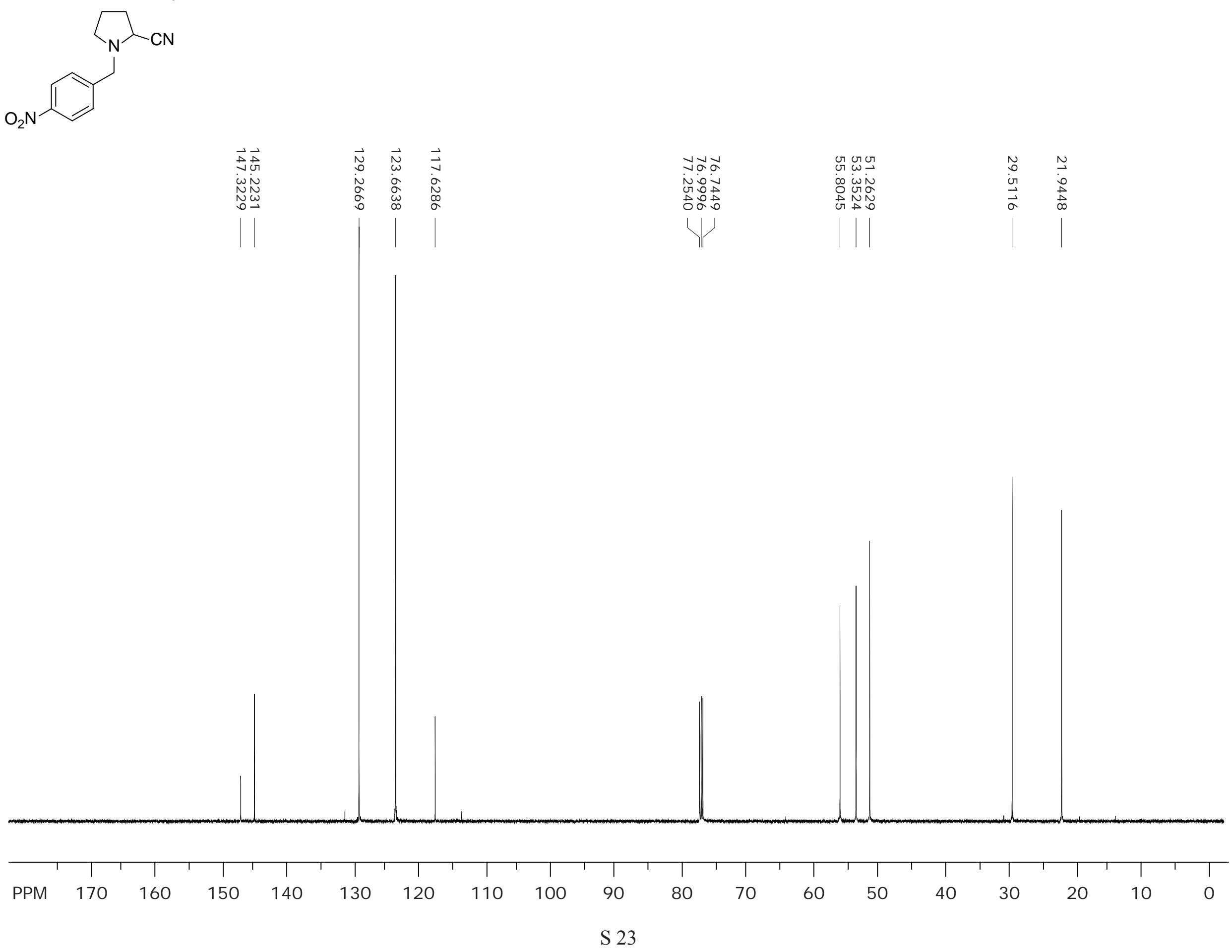
${ }^{1} \mathrm{H}$ NMR of $\mathbf{4}_{\mathbf{j}}$ in $\mathrm{CDCl}_{3}$
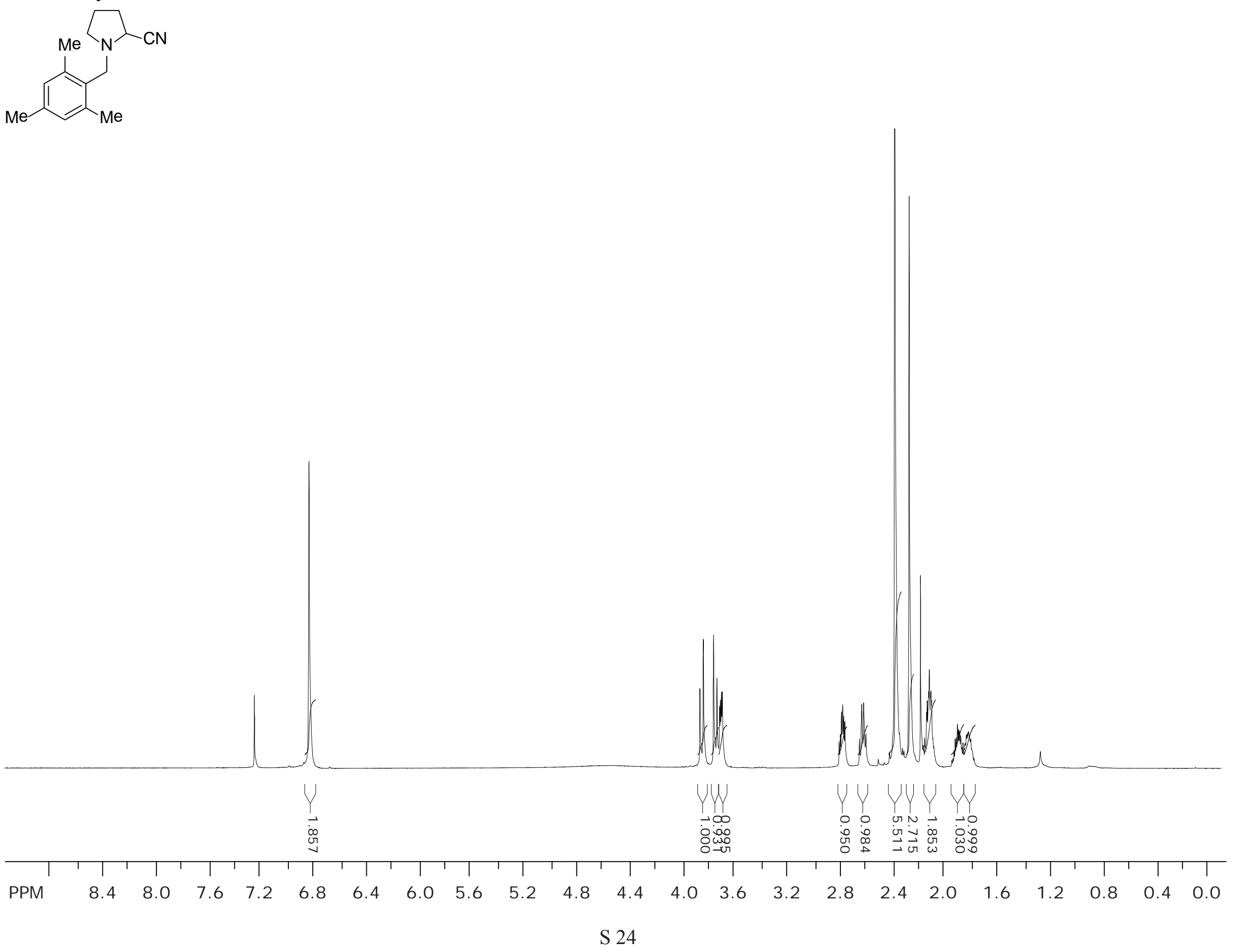
${ }^{13} \mathrm{C} \mathrm{NMR}$ of $\mathbf{4} \mathbf{j}$ in $\mathrm{CDCl}_{3}$
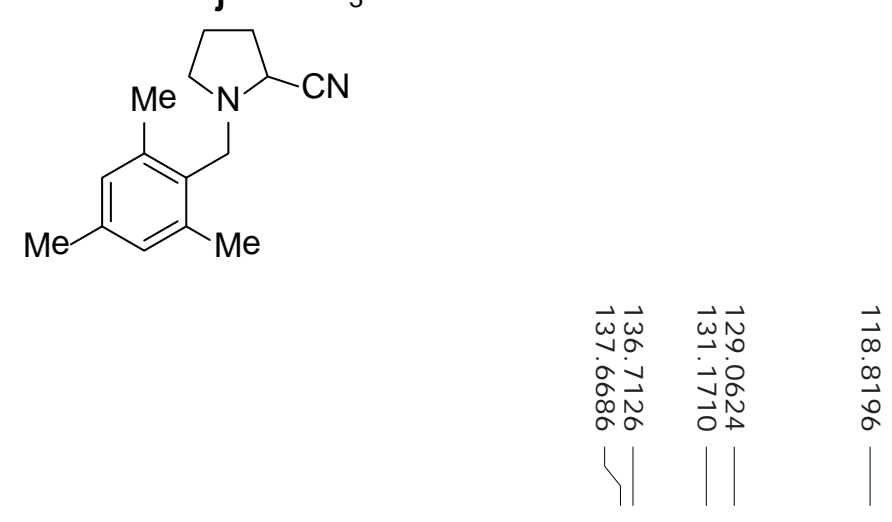

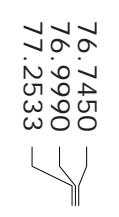
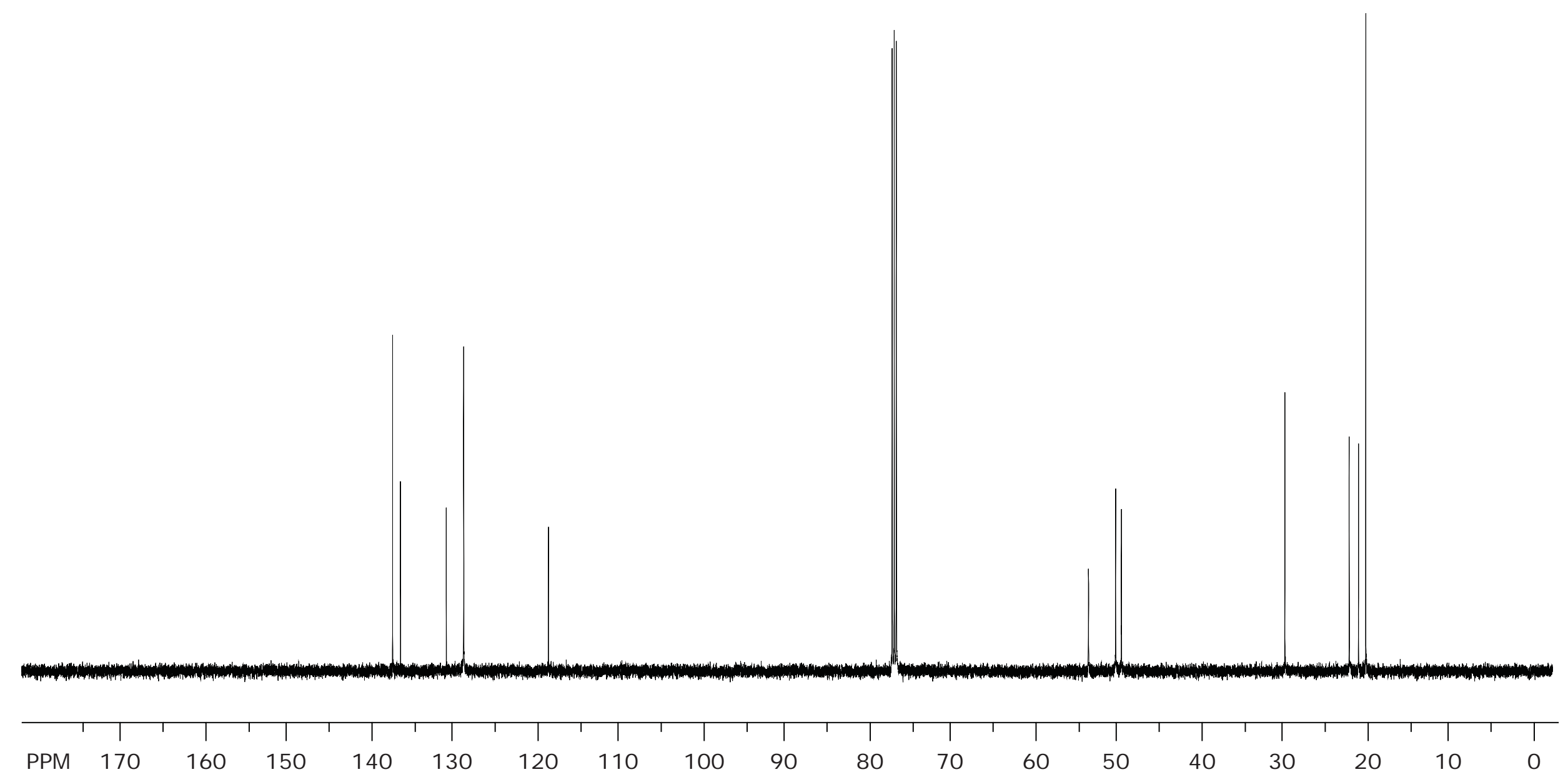
${ }^{1} \mathrm{H} \mathrm{NMR}$ of $\mathbf{4 k}$ in $\mathrm{CDCl}_{3}$
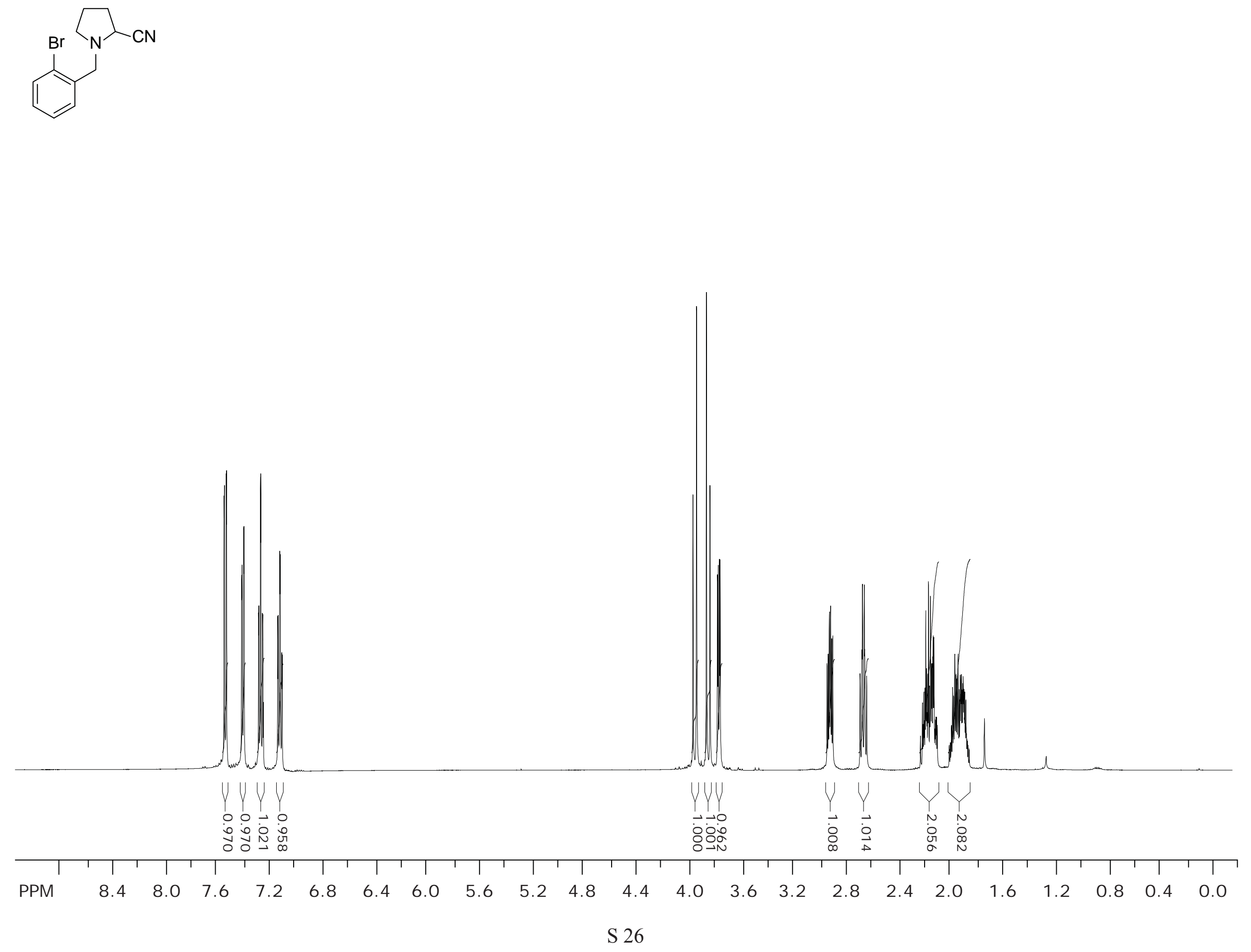
${ }^{13} \mathrm{C} \mathrm{NMR}$ of $\mathbf{4 k}$ in $\mathrm{CDCl}_{3}$
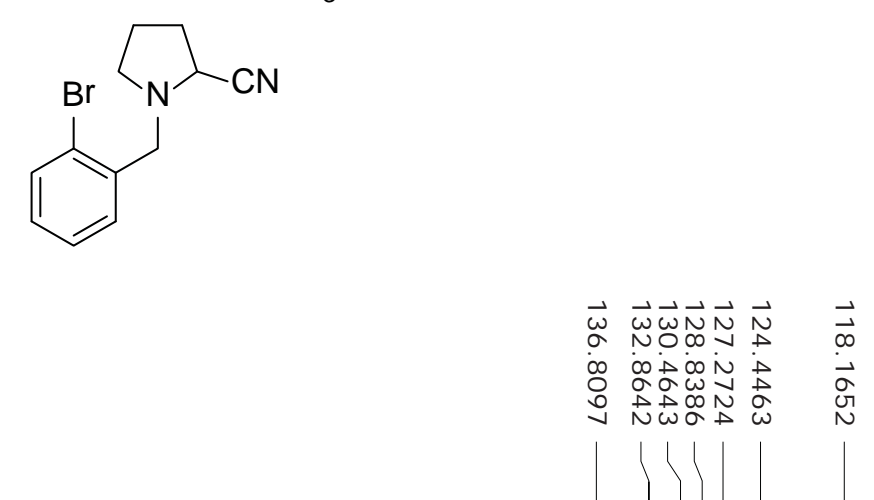

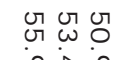

웅ㅎํ

w

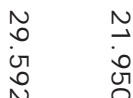

जั०ं

जูำ

$\mid$

ज
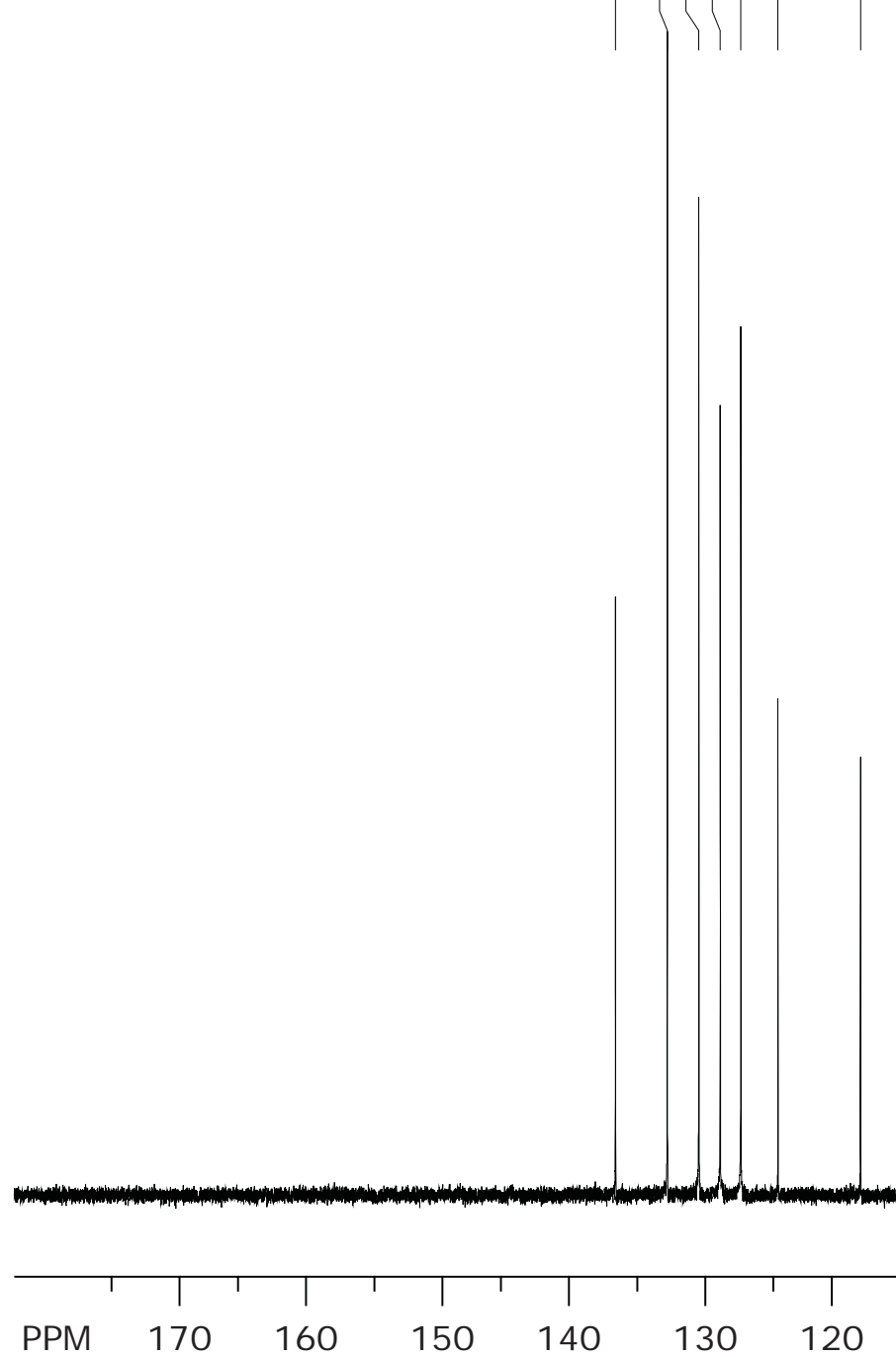

110

100

90

80

70

60

50

40

30

20

10 
${ }^{1} \mathrm{H}$ NMR of $4 \mathrm{l}$ in $\mathrm{CDCl}_{3}$
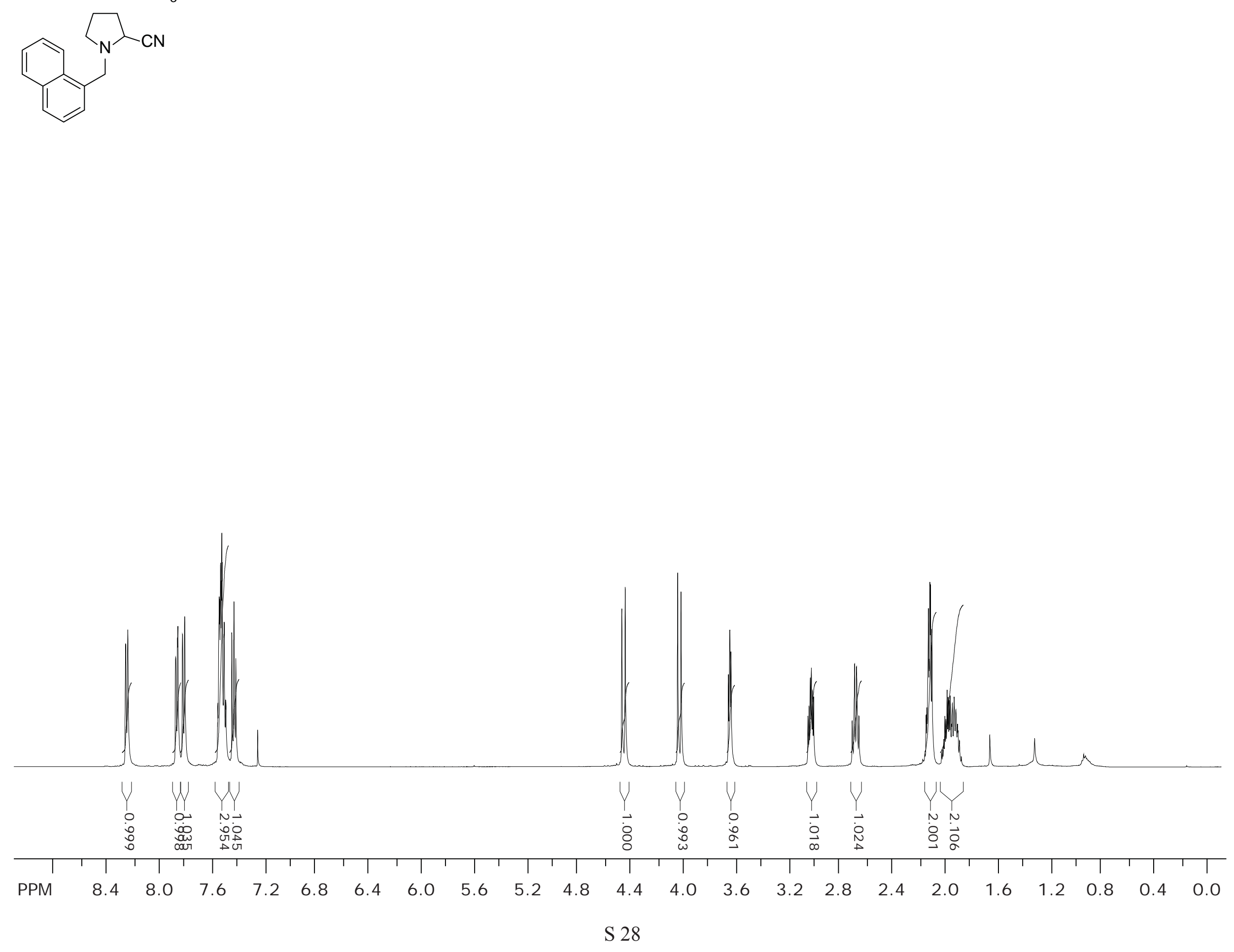
${ }^{13} \mathrm{C}$ NMR of $4 \mathrm{I}$ in $\mathrm{CDCl}_{3}$
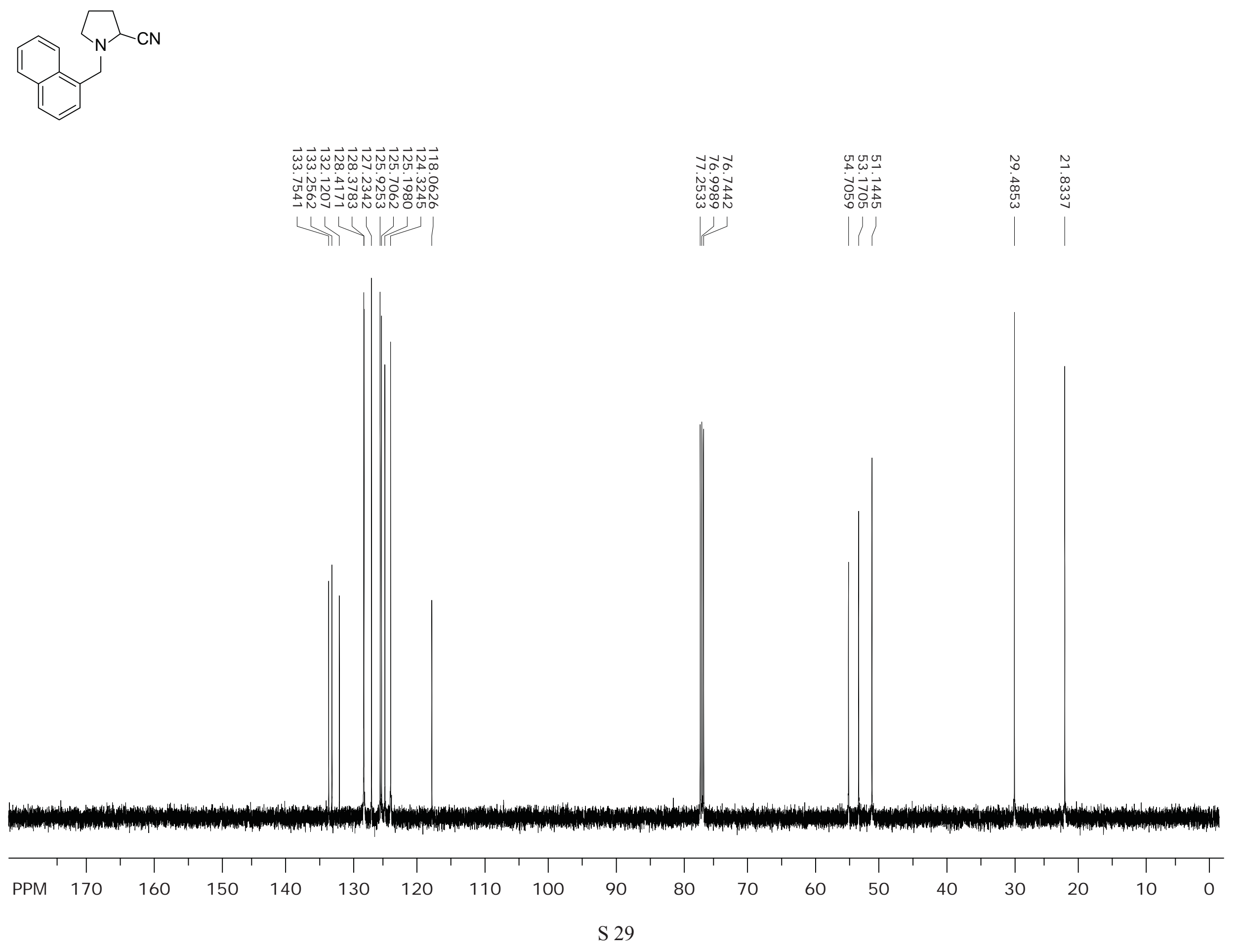
${ }^{1} \mathrm{H}$ NMR of $4 \mathrm{~m}$ in $\mathrm{CDCl}_{3}$
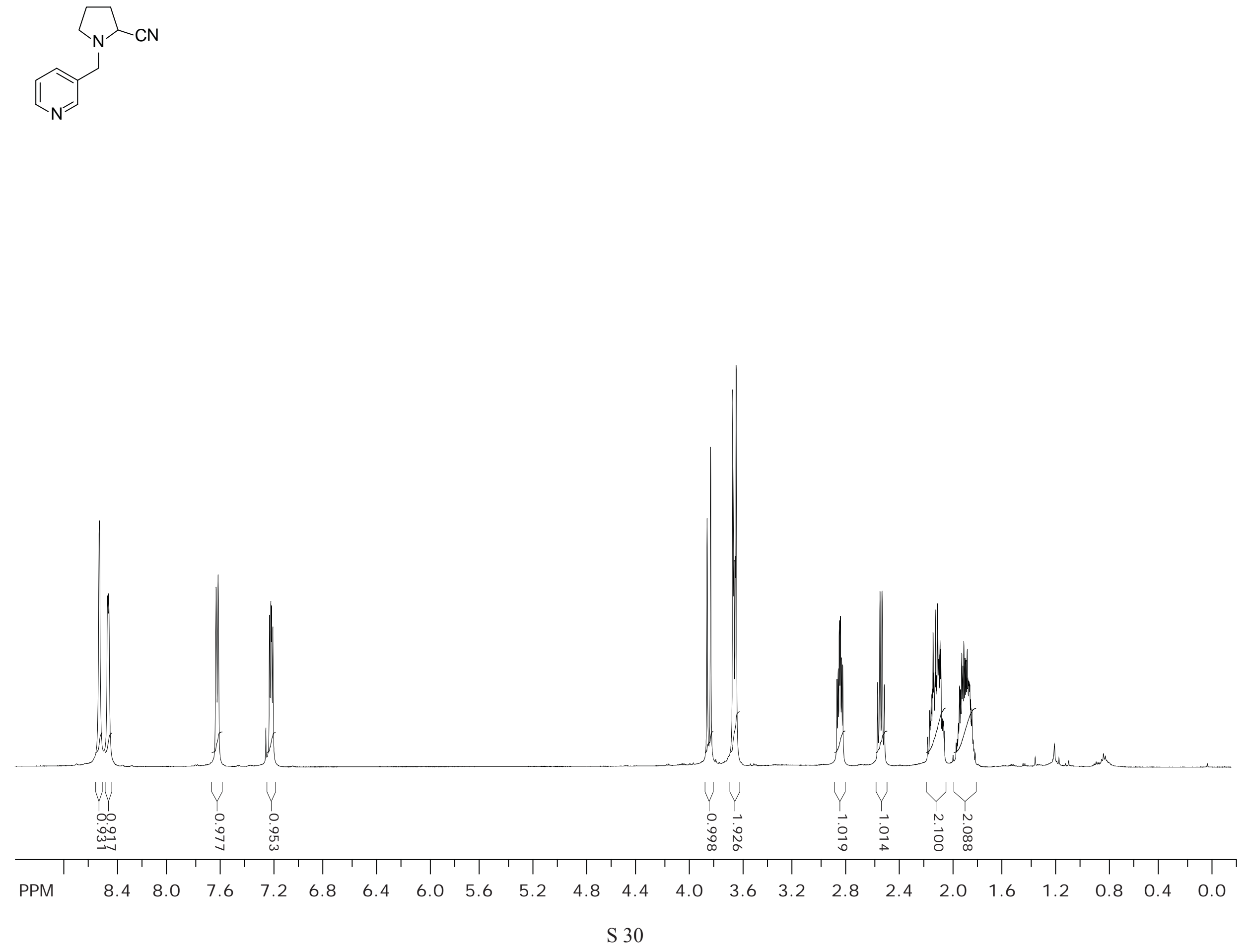
${ }^{13} \mathrm{C} \mathrm{NMR}$ of $\mathbf{4 m}$ in $\mathrm{CDCl}_{3}$
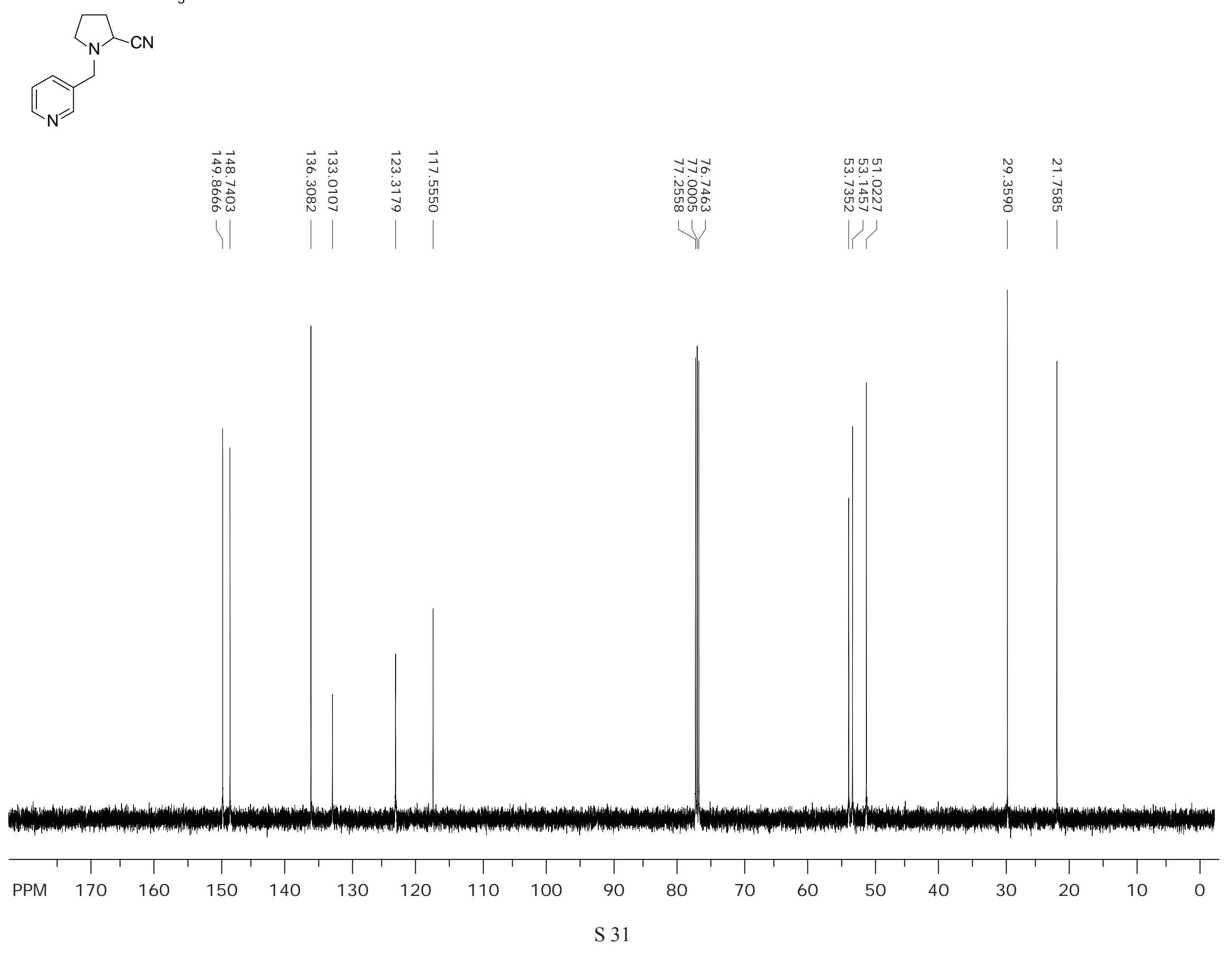
${ }^{1} \mathrm{H}$ NMR of $\mathbf{4 n}$ in $\mathrm{CDCl}_{3}$
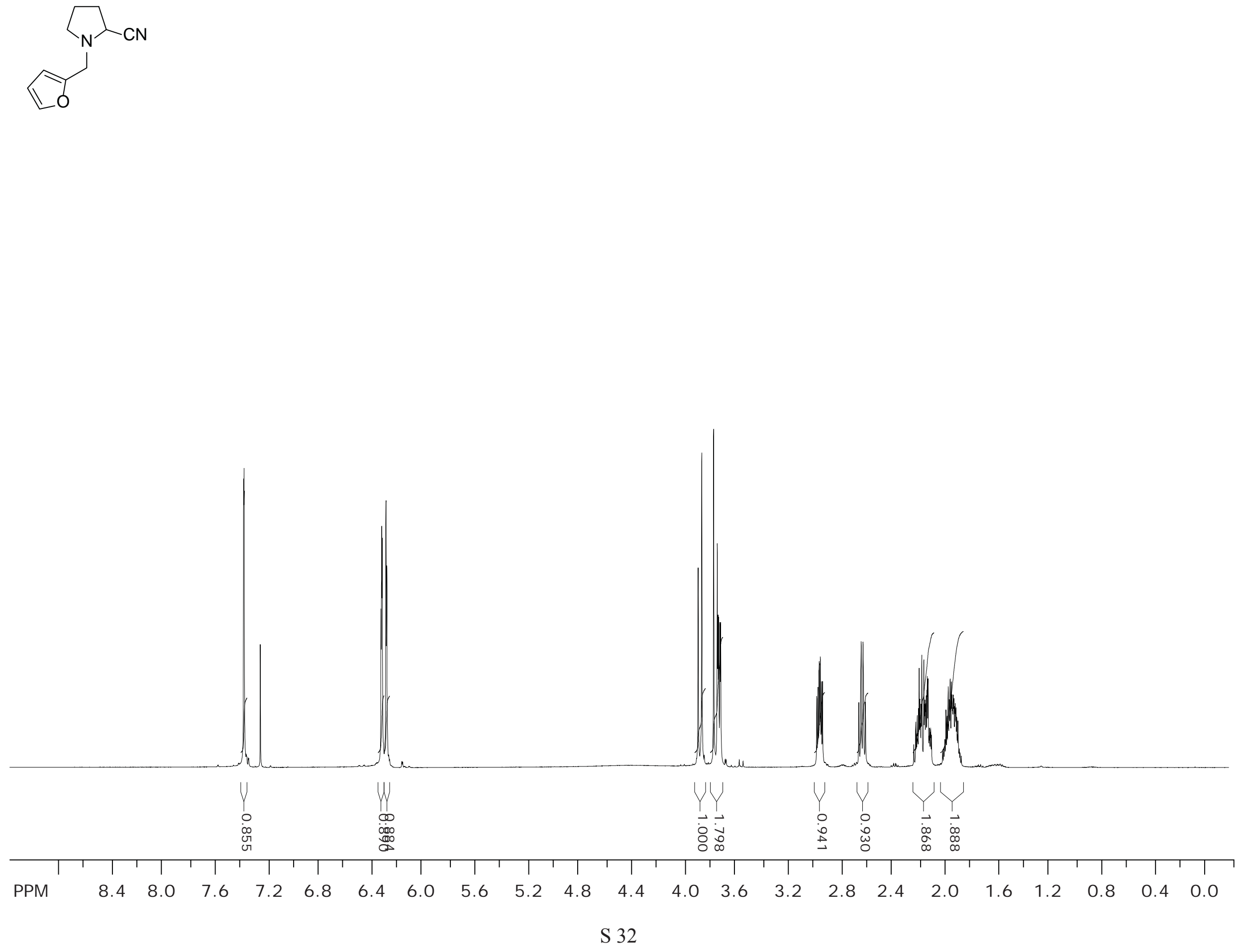
${ }^{13} \mathrm{C} \mathrm{NMR}$ of $\mathbf{4 n}$ in $\mathrm{CDCl}_{3}$
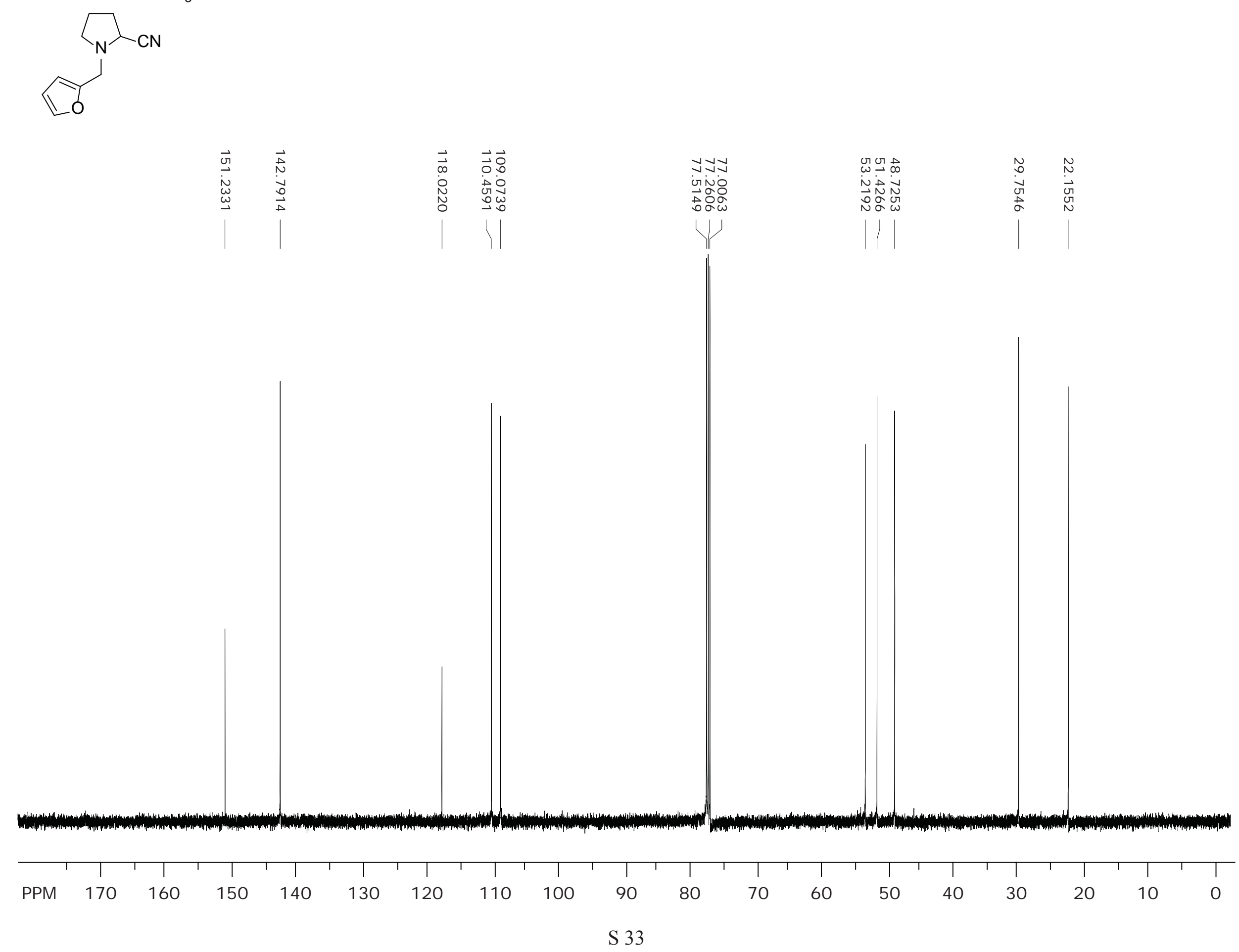
${ }^{1} \mathrm{H}$ NMR of 40 in $\mathrm{CDCl}_{3}$<smiles>N#CC1CCCN1</smiles>

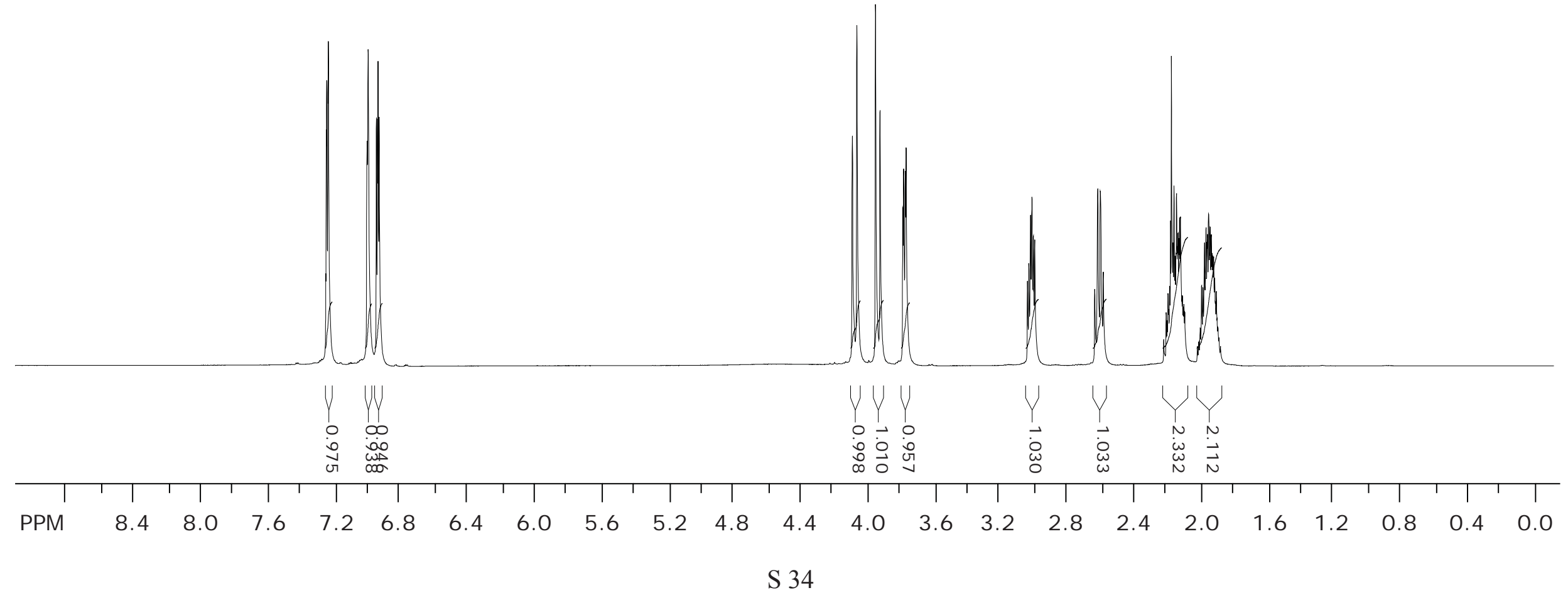


${ }^{13} \mathrm{C}$ NMR of 40 in $\mathrm{CDCl}_{3}$
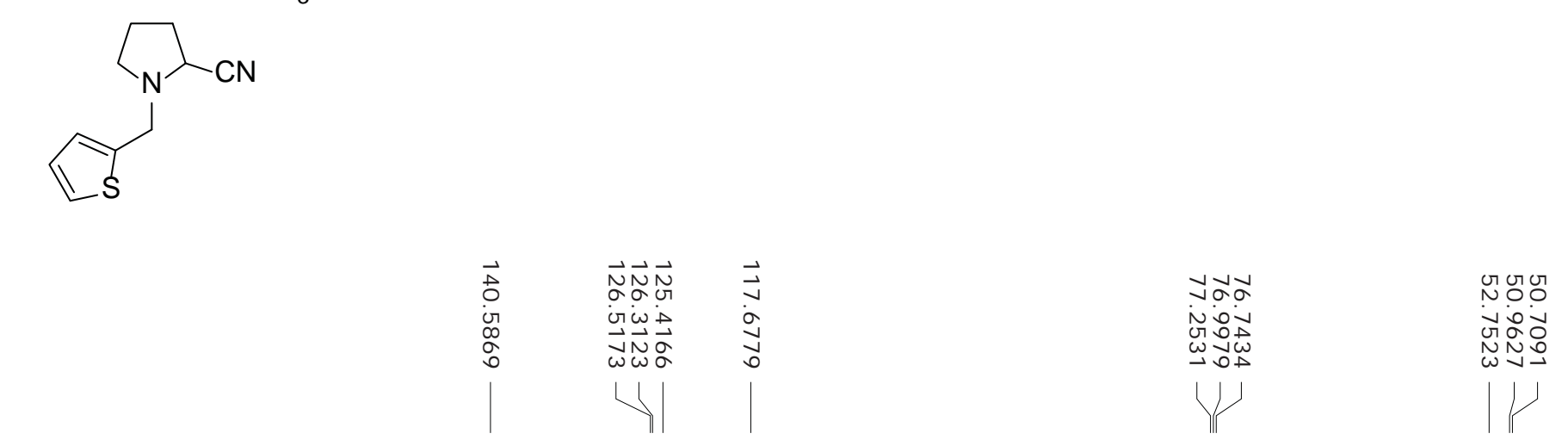

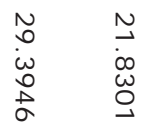
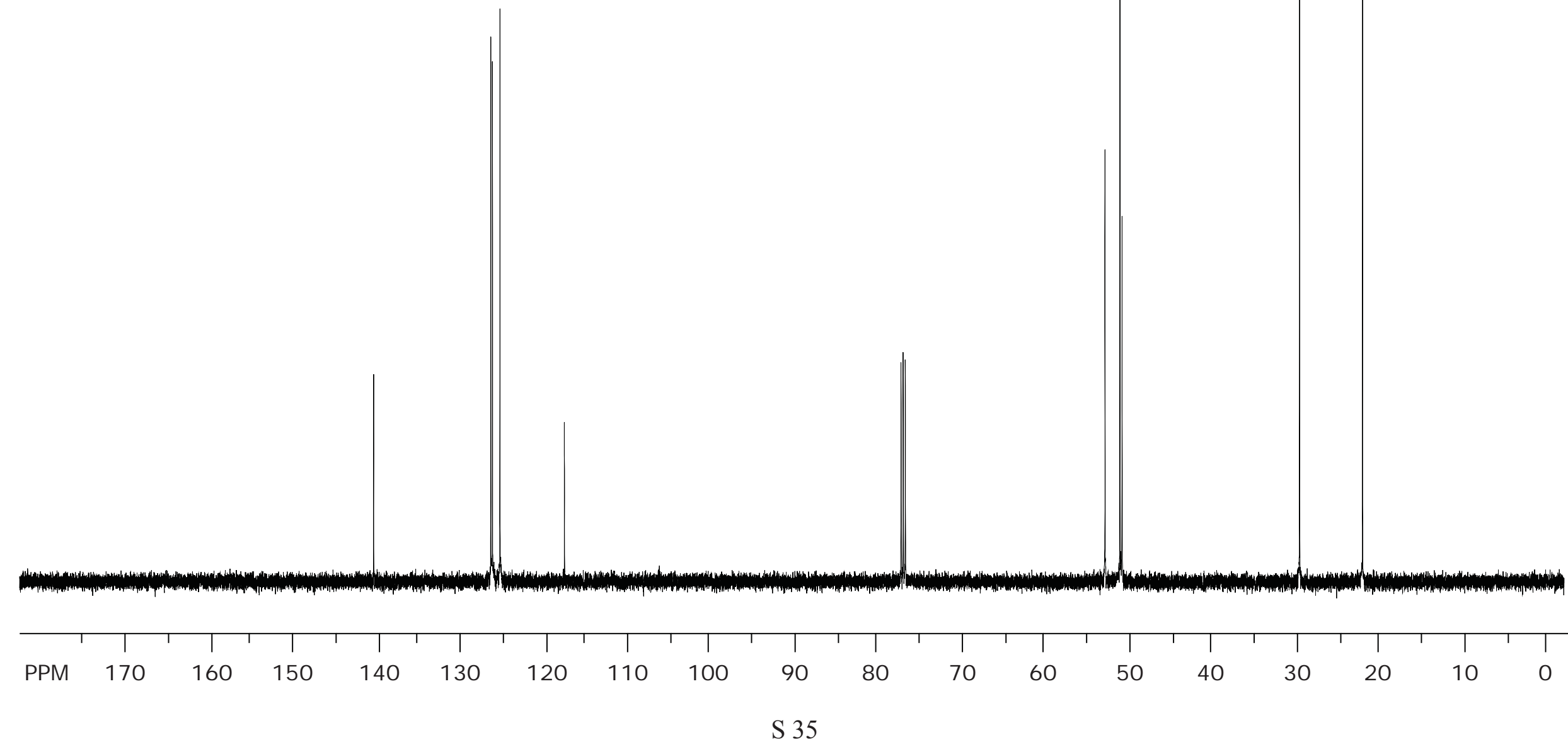


\section{${ }^{1} \mathrm{H} \mathrm{NMR}$ of $4 \mathrm{p}$ in $\mathrm{CDCl}_{3}$}
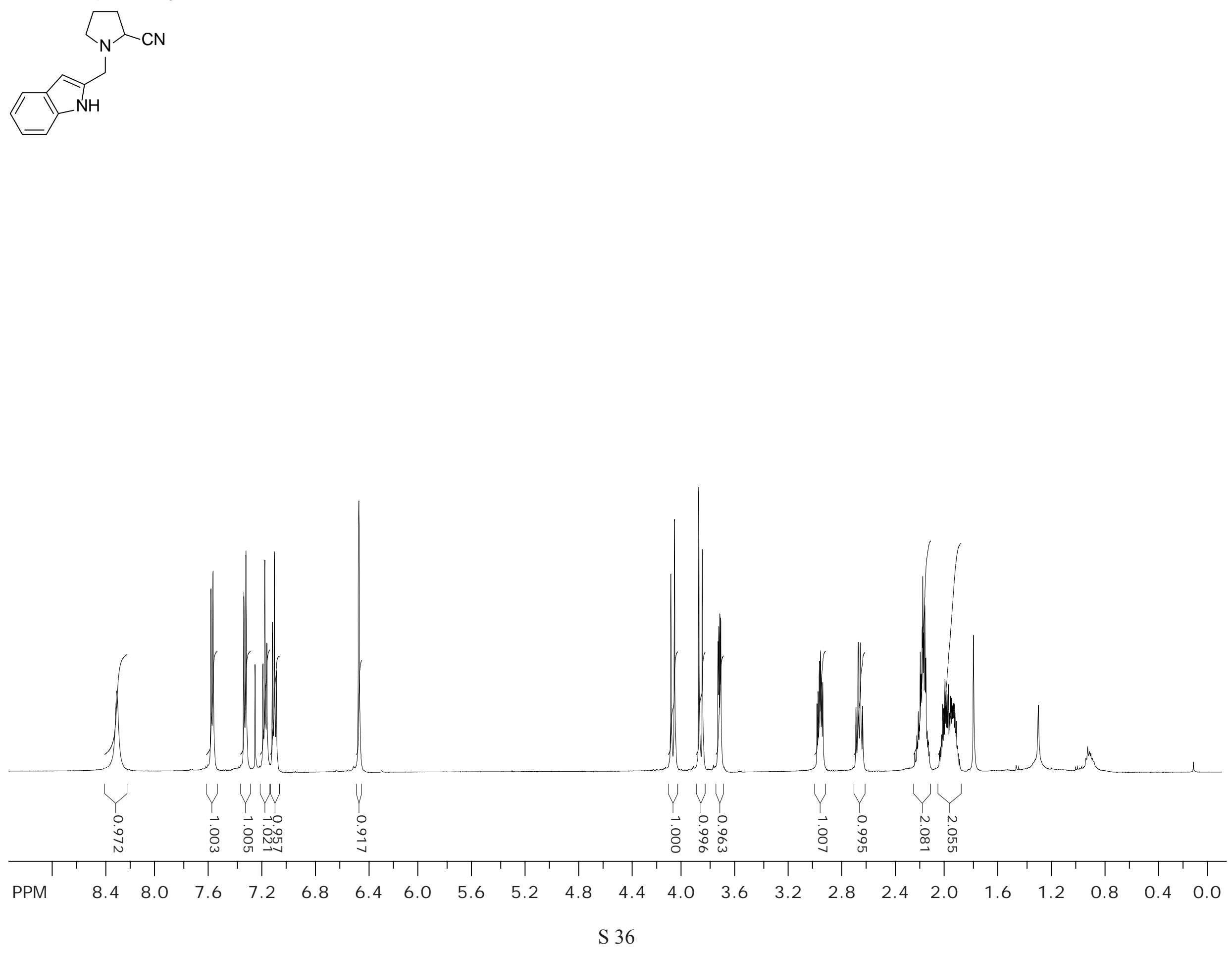
${ }^{13} \mathrm{C} \mathrm{NMR}$ of $\mathbf{4} \mathbf{p}$ in $\mathrm{CDCl}_{3}$

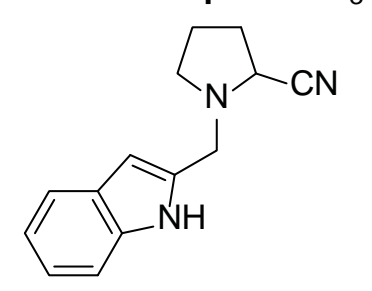

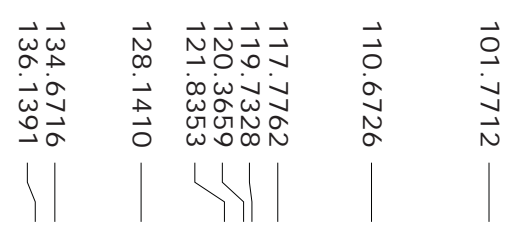

ㅊำ

जi:

무에

H

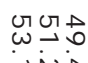

on

年

$1 \mid$

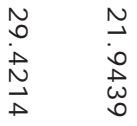

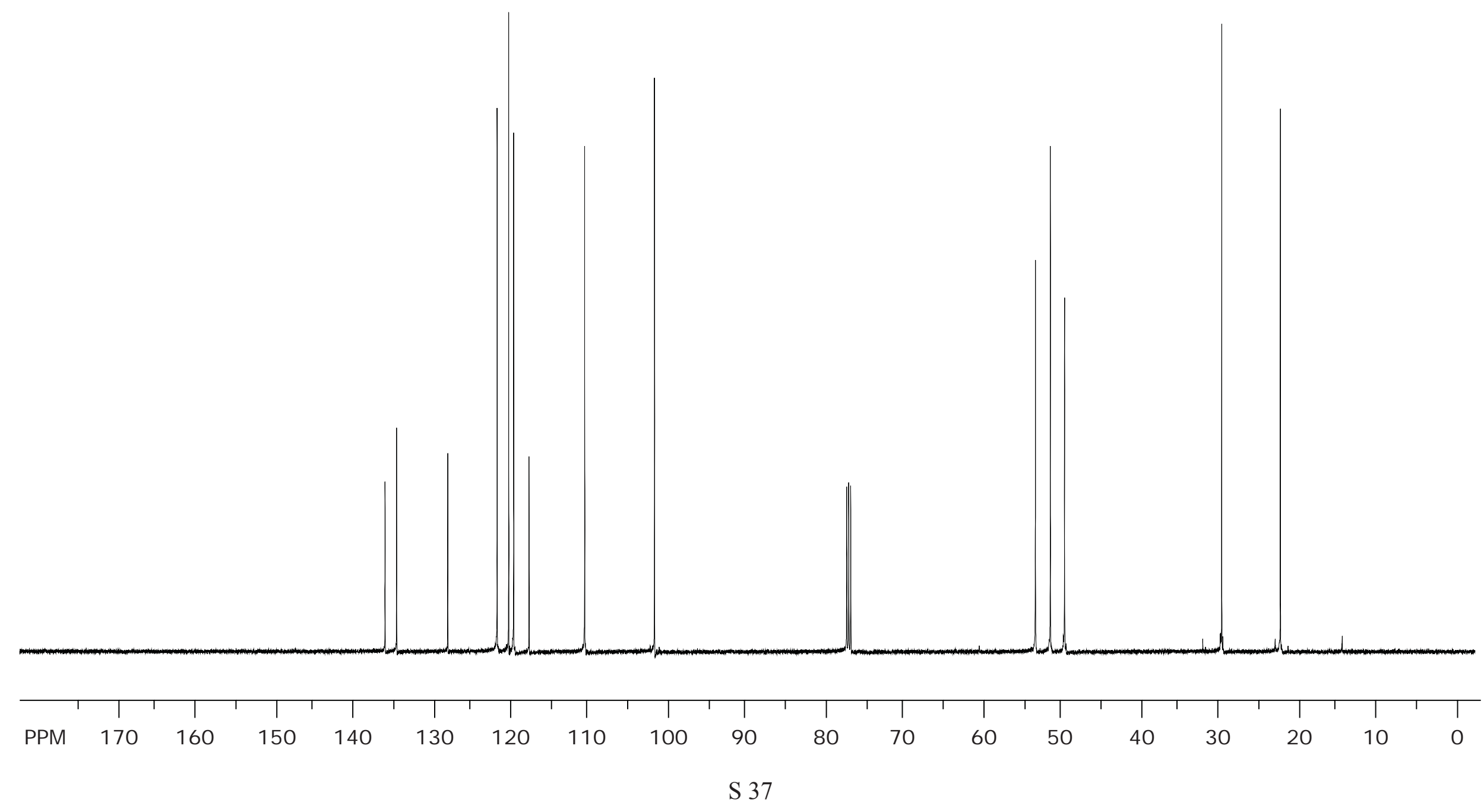


${ }^{1} \mathrm{H}$ NMR of $\mathbf{4} \mathbf{q}$ in $\mathrm{CDCl}_{3}$

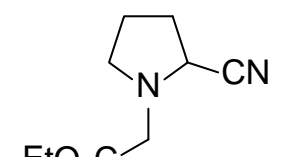

$\mathrm{EtO}_{2} \mathrm{C}$

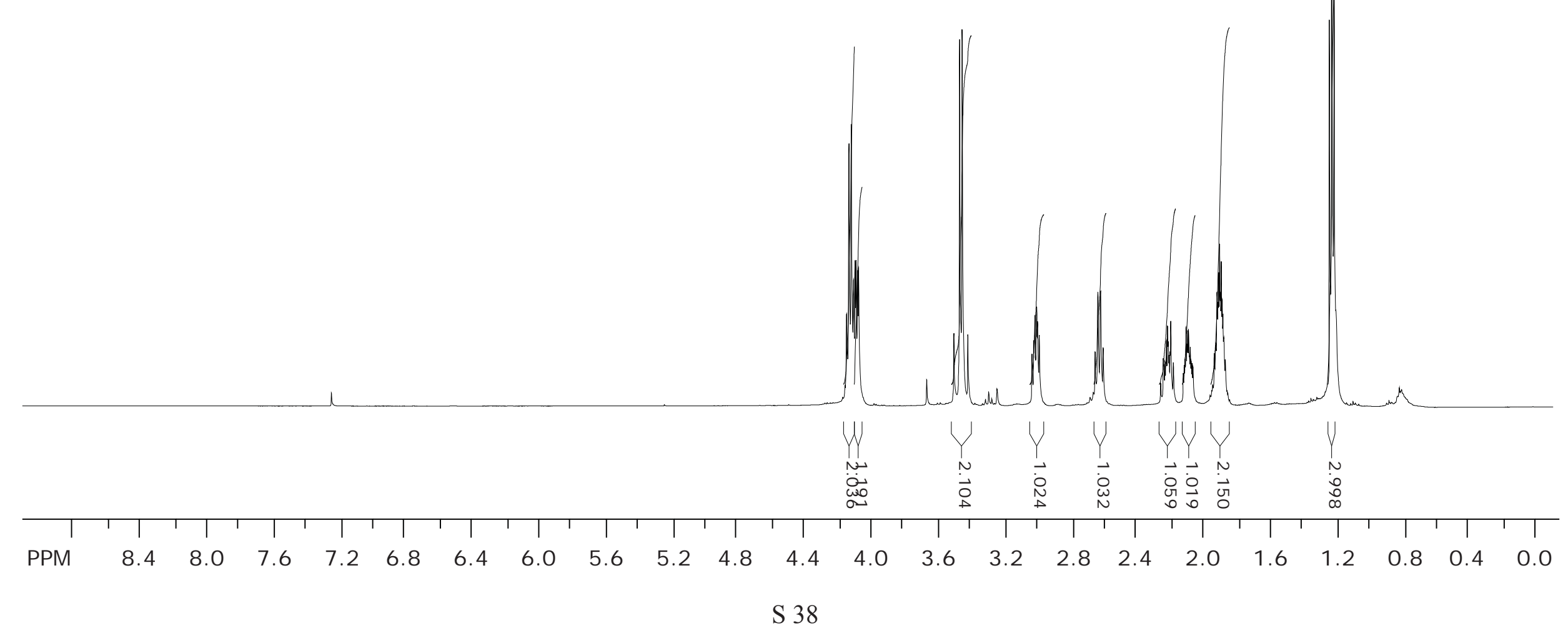


${ }^{13} \mathrm{C} \mathrm{NMR}$ of $\mathbf{4 q}$ in $\mathrm{CDCl}_{3}$

$$
\mathrm{EtO}_{2} \mathrm{C}
$$

\begin{tabular}{|c|c|c|c|c|c|c|}
\hline 苟 & $\begin{array}{l}\stackrel{⿱}{\Delta} \\
\dot{0} \\
\stackrel{\sim}{N} \\
\omega\end{array}$ & 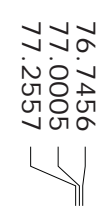 & $\begin{array}{l}0 \\
8 \\
8 \\
0 \\
0\end{array}$ & 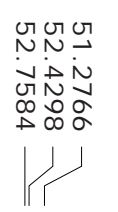 & $\begin{array}{l}\tilde{0} \\
\dot{U} \\
0 \\
\bullet\end{array}$ & \\
\hline
\end{tabular}

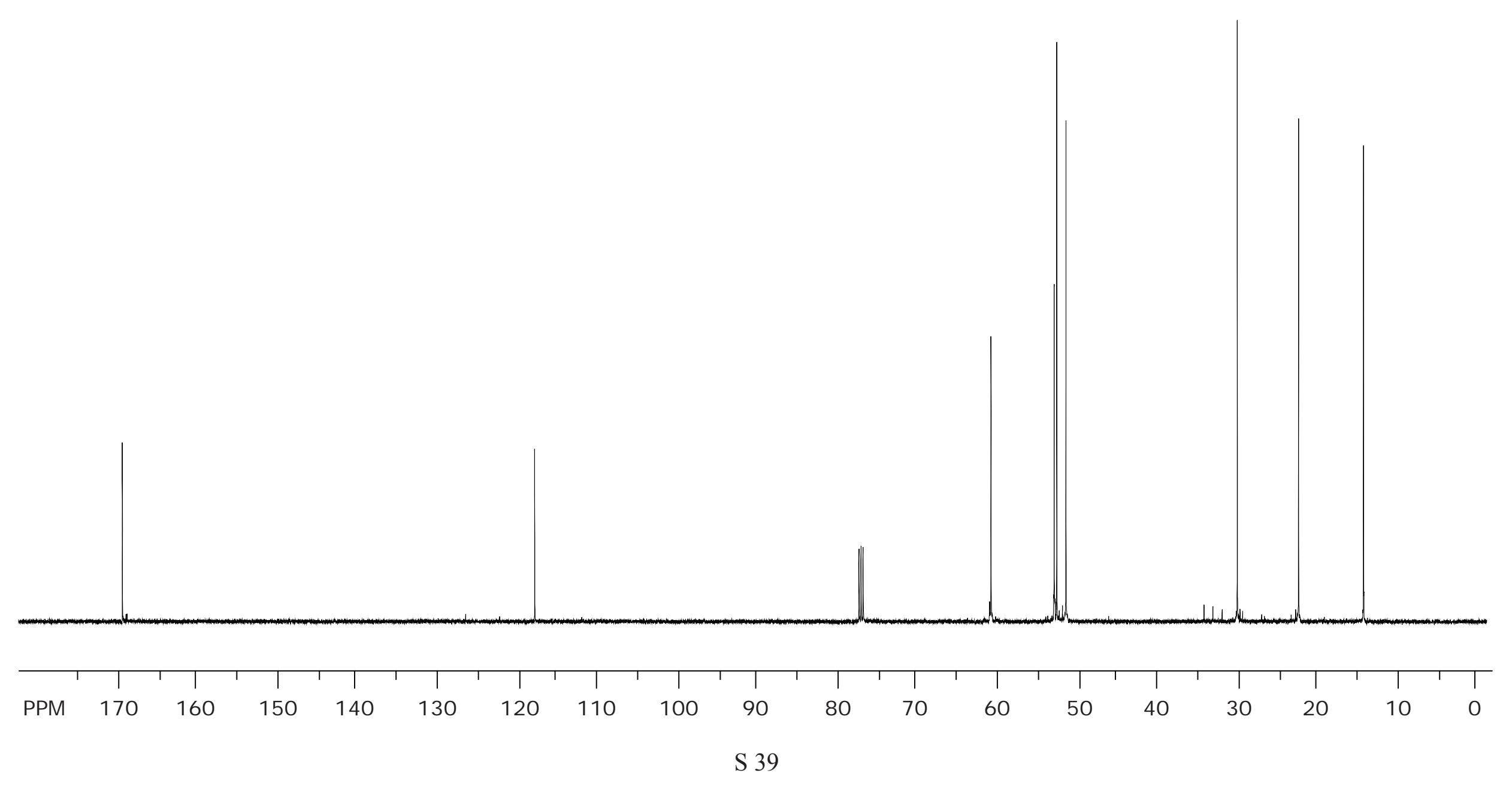




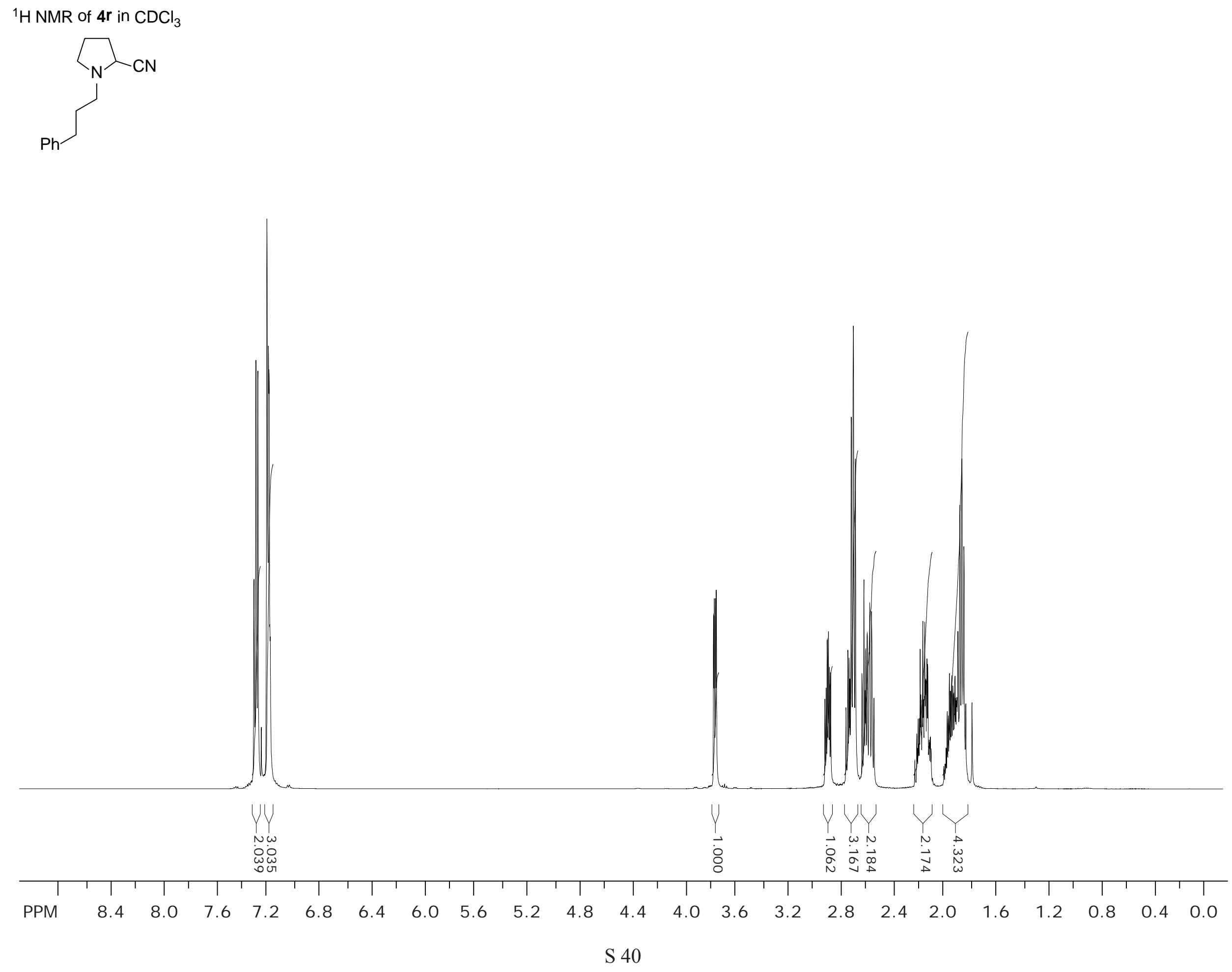


${ }^{13} \mathrm{C}$ NMR of $4 \mathbf{r}$ in $\mathrm{CDCl}_{3}$
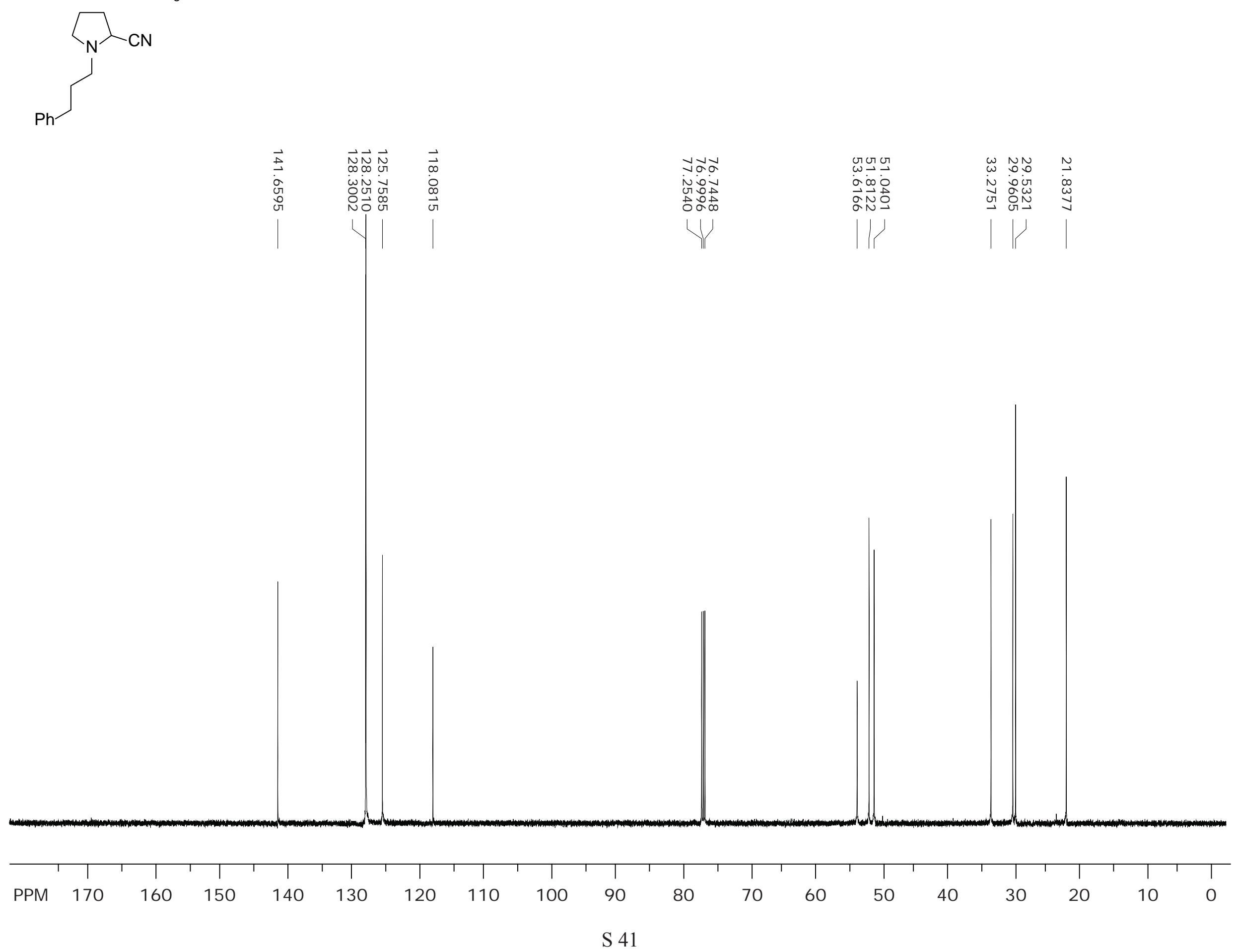
${ }^{1} \mathrm{H} \mathrm{NMR}$ of $4 \mathrm{~s}$ in $\mathrm{CDCl}_{3}$<smiles>N#CC1CCCN1CCc1ccccc1</smiles>

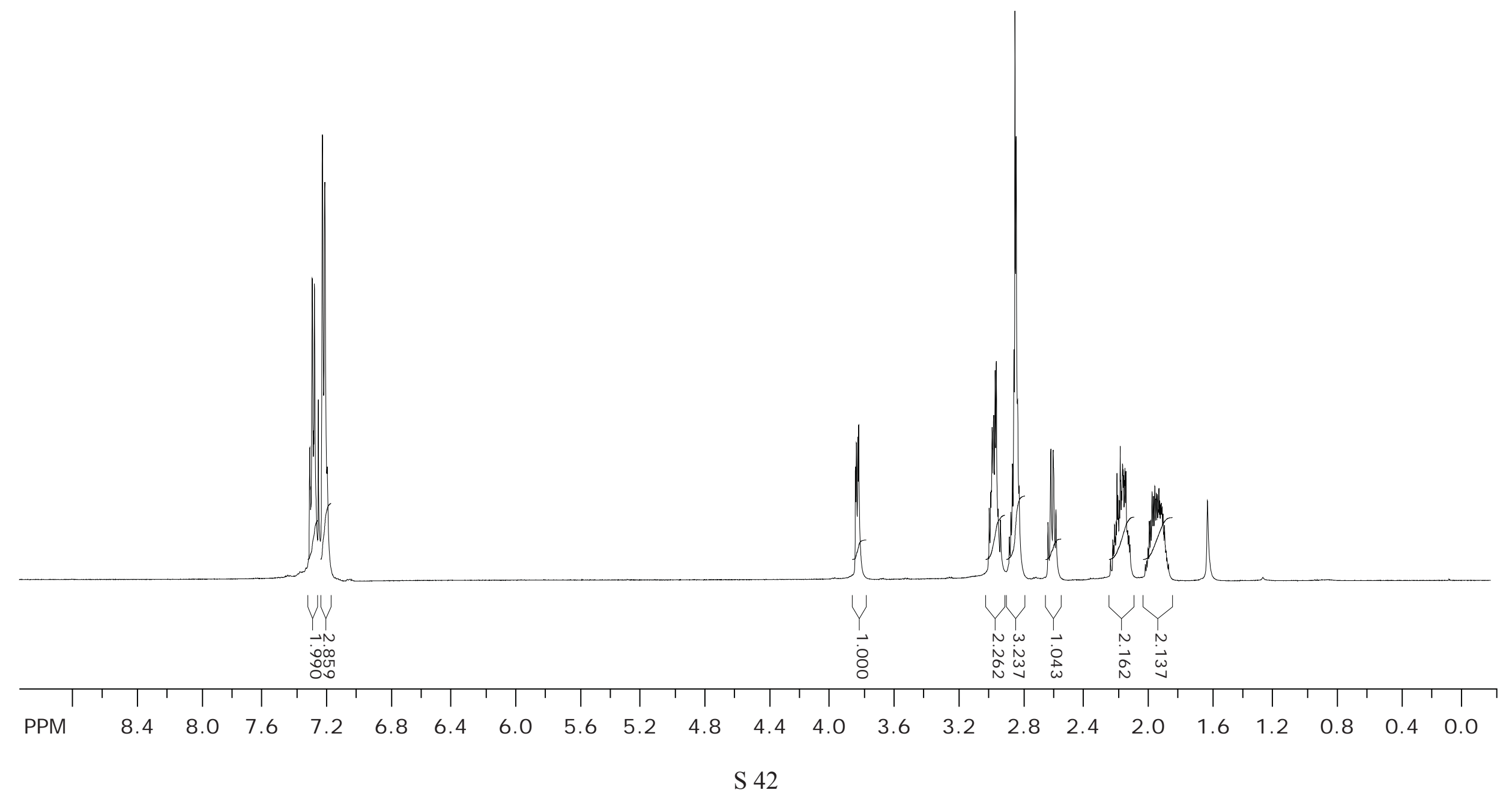


${ }^{13} \mathrm{C}$ NMR of $4 \mathrm{~s}$ in $\mathrm{CDCl}_{3}$<smiles>N#CC1CCCN1CCc1ccccc1</smiles>
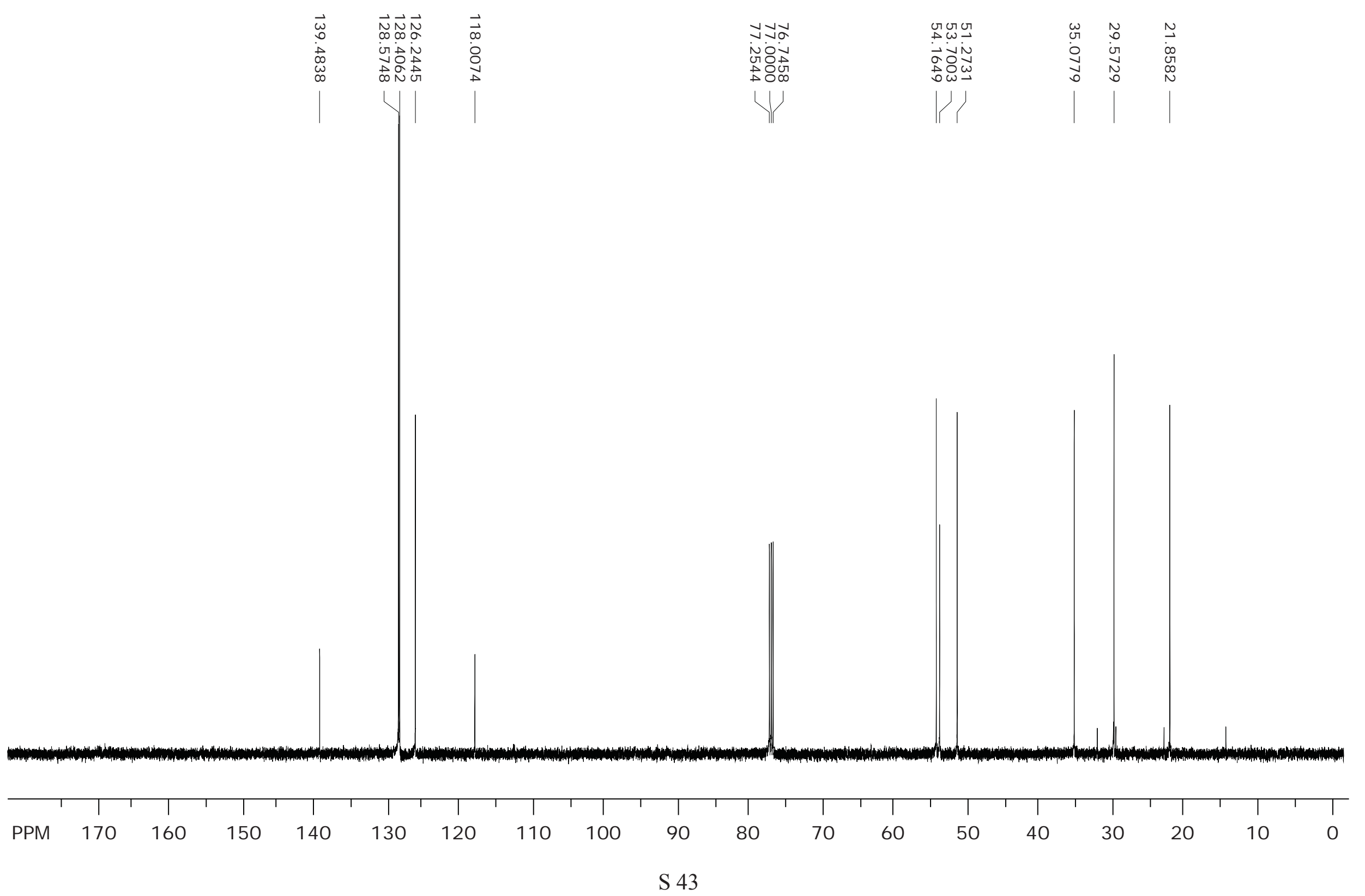
${ }^{1} \mathrm{H} \mathrm{NMR}$ of $4 \mathrm{t}$ in $\mathrm{CDCl}_{3}$
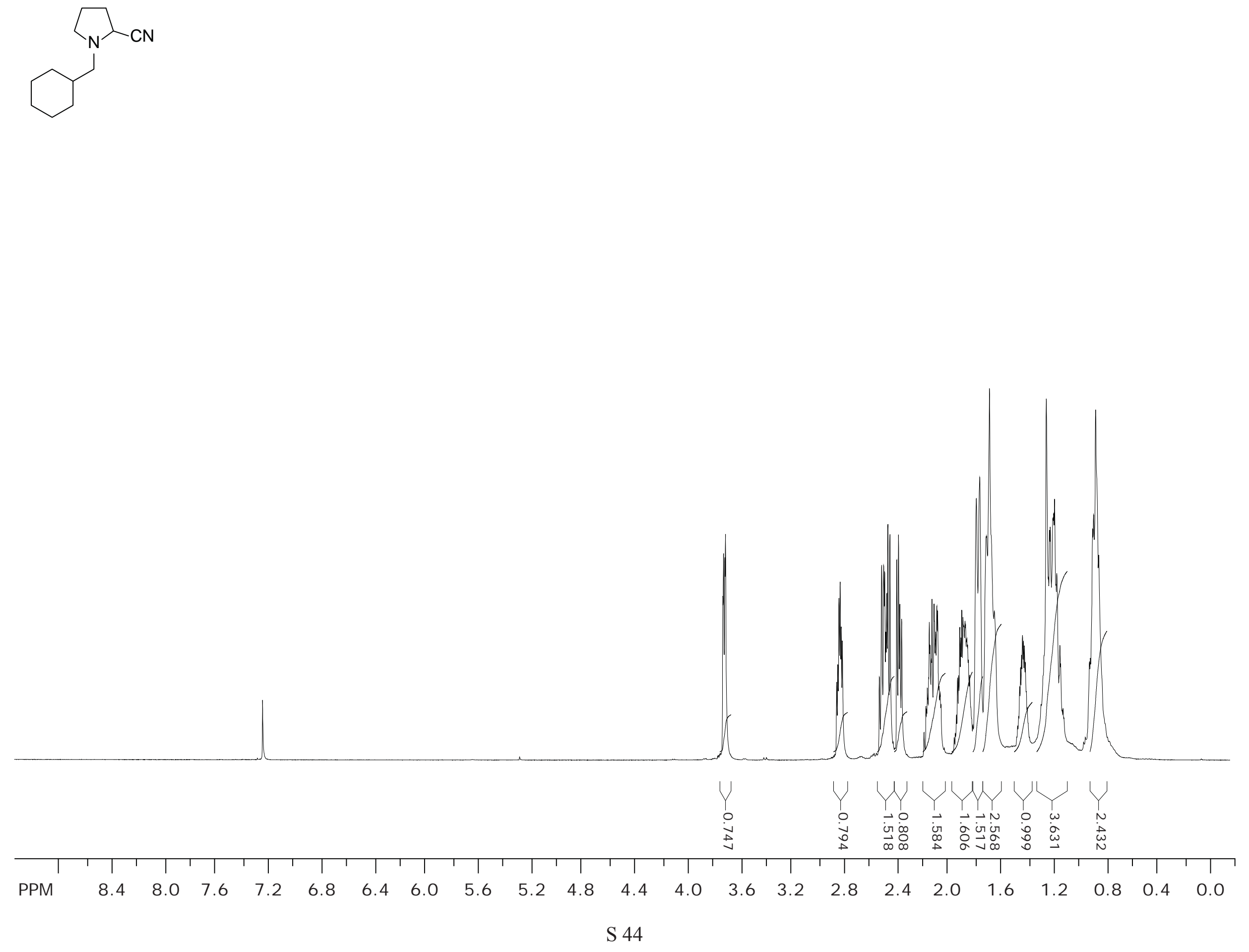
${ }^{13} \mathrm{C} \mathrm{NMR}$ of $\mathbf{4 t}$ in $\mathrm{CDCl}_{3}$
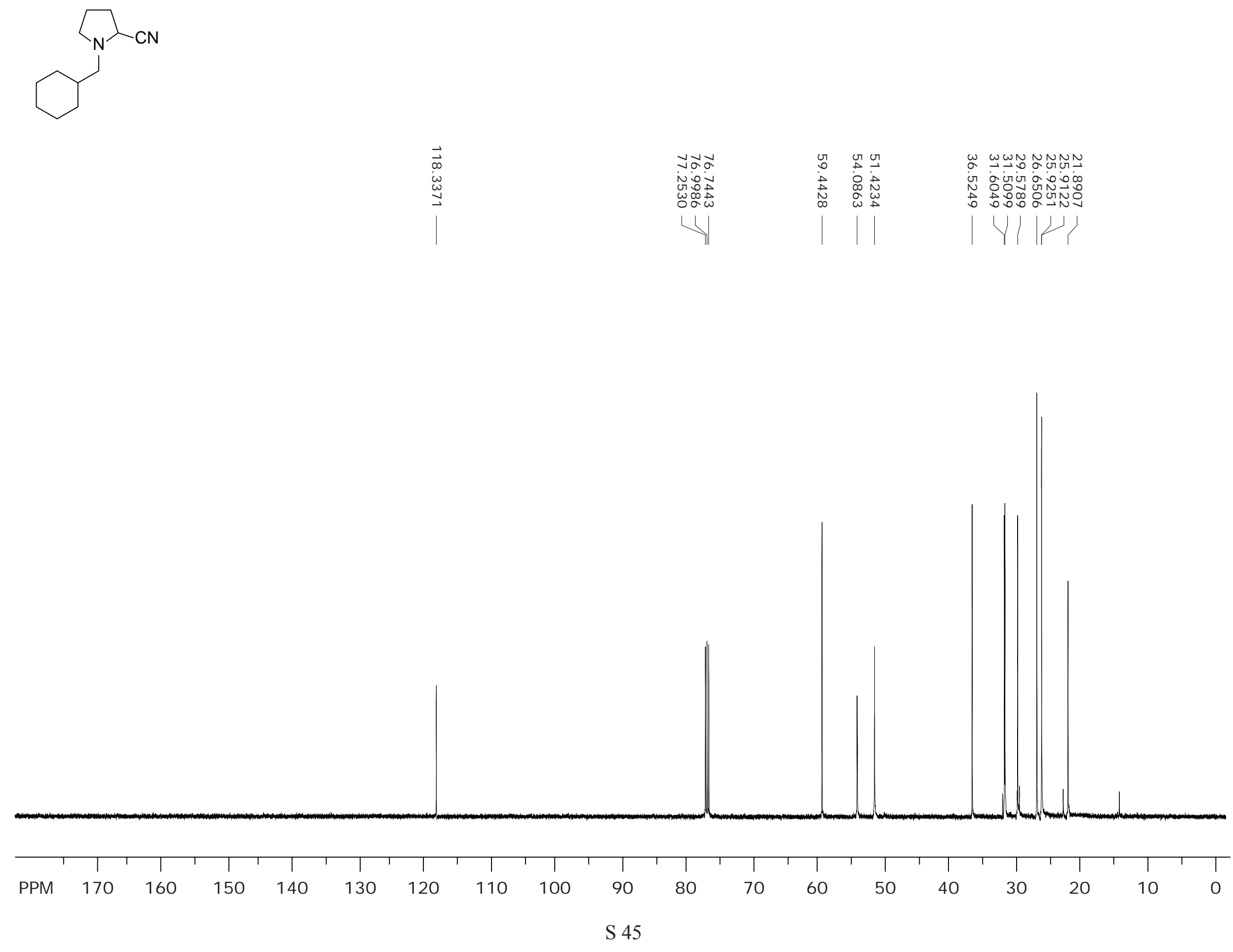
${ }^{1} \mathrm{H} \mathrm{NMR}$ of $\mathbf{4 u}$ in $\mathrm{CDCl}_{3}$
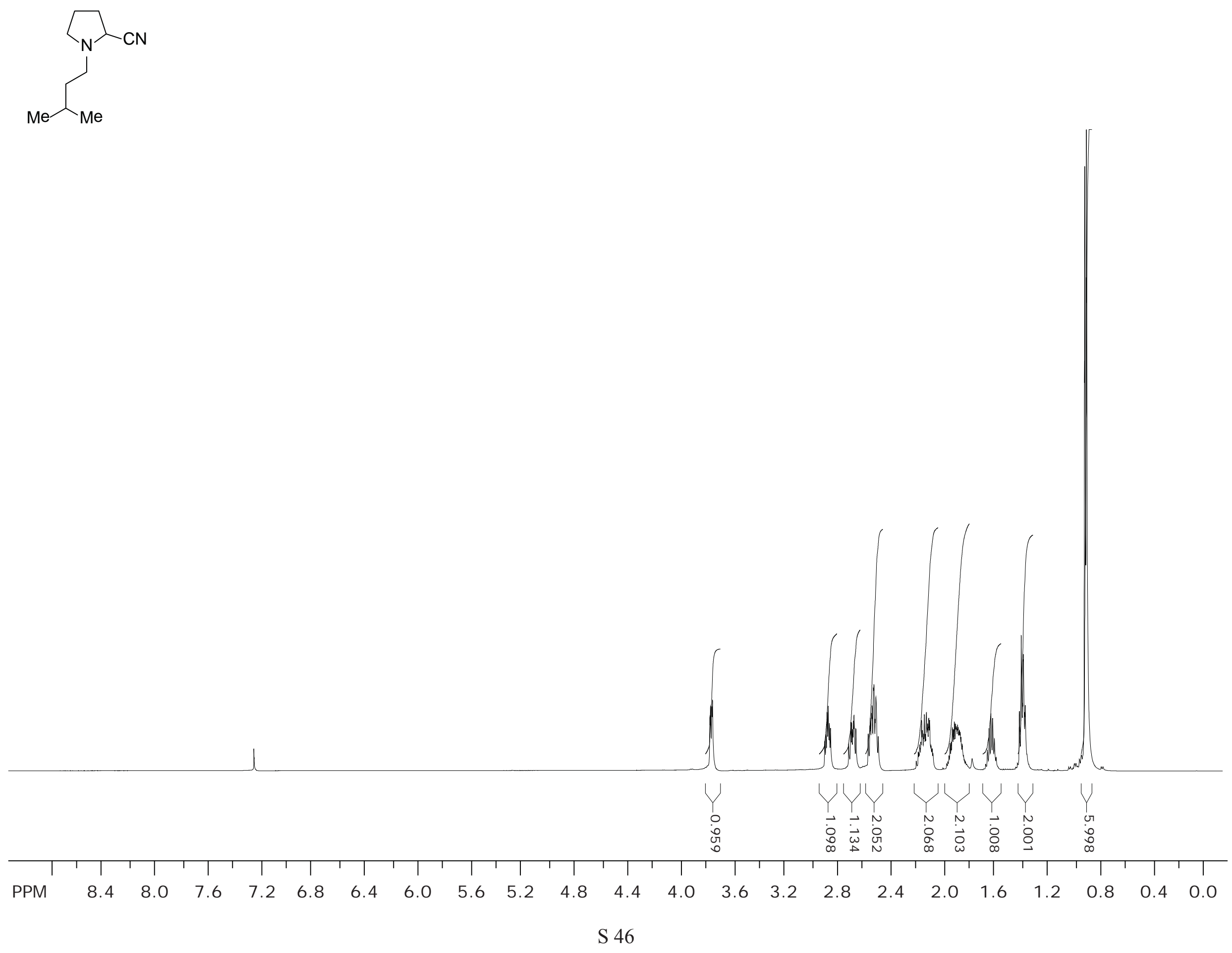
${ }^{13} \mathrm{C}$ NMR of $4 \mathbf{u}$ in $\mathrm{CDCl}_{3}$
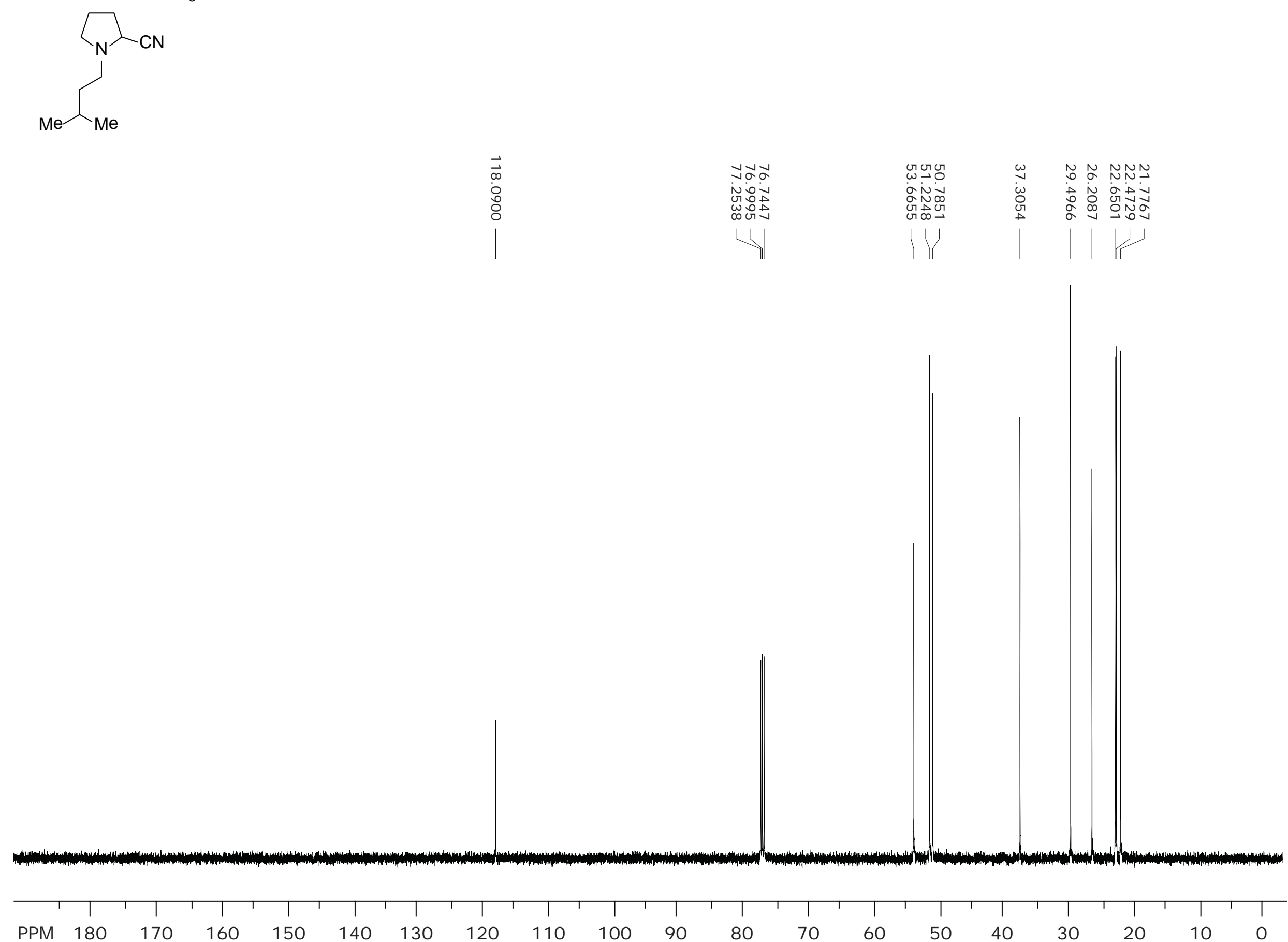
${ }^{1} \mathrm{H}$ NMR of $\mathbf{4} \mathbf{v}$ in $\mathrm{CDCl}_{3}$
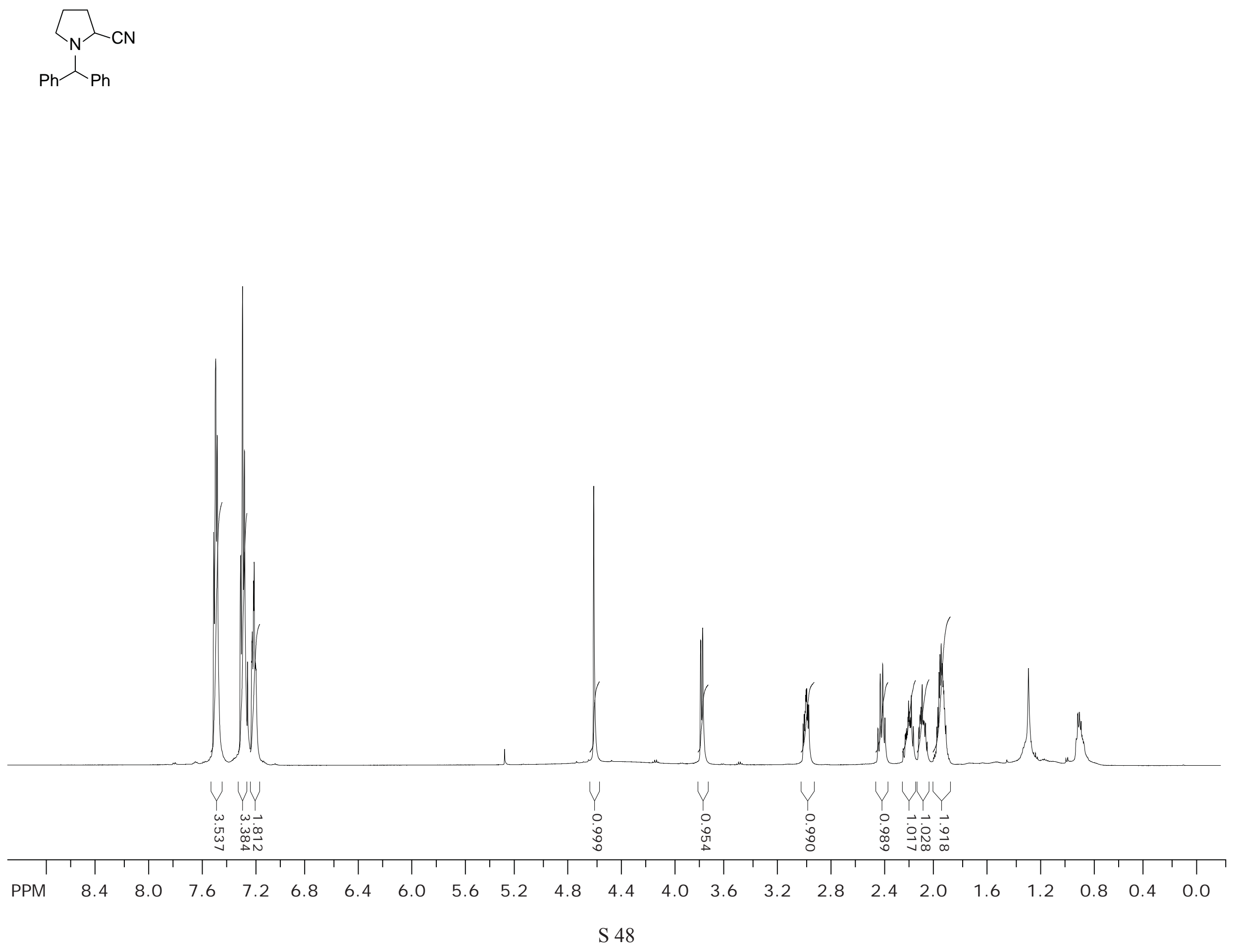
${ }^{13} \mathrm{C}$ NMR of $\mathbf{4} \mathbf{v}$ in $\mathrm{CDCl}_{3}$
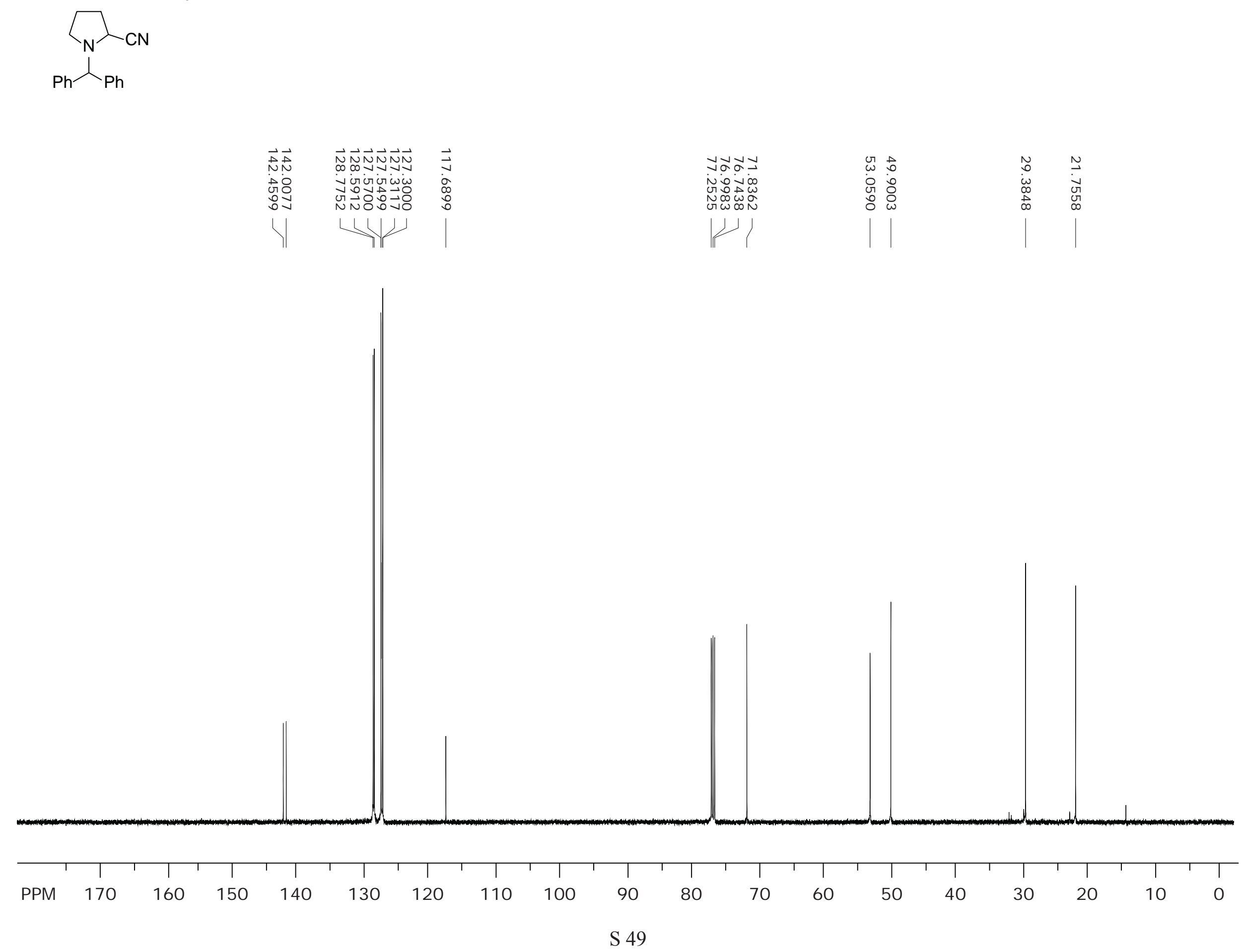
${ }^{1} \mathrm{H}$ NMR of 7 in $\mathrm{CDCl}_{3}$
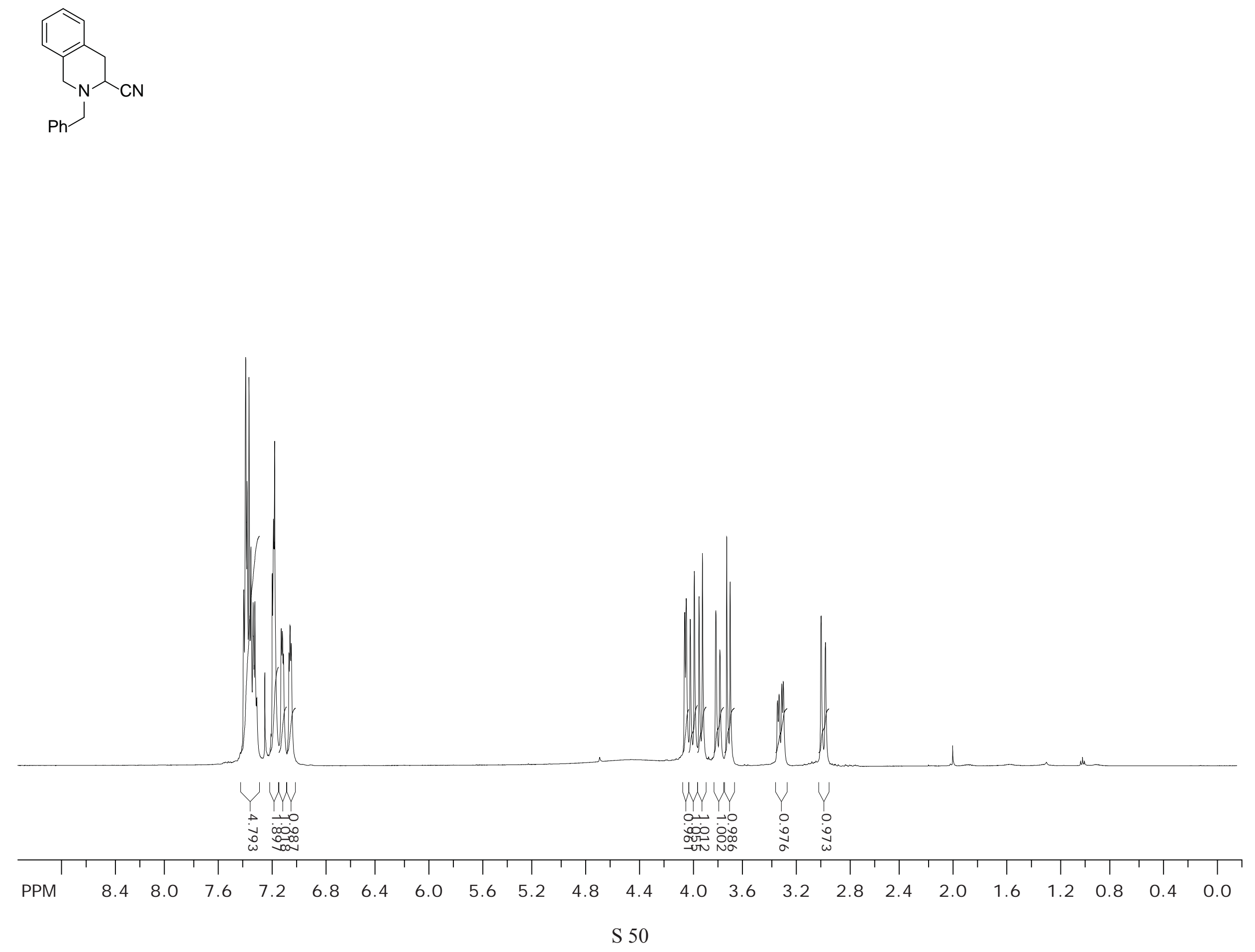
${ }^{13} \mathrm{C}$ NMR of 7 in $\mathrm{CDCl}_{3}$
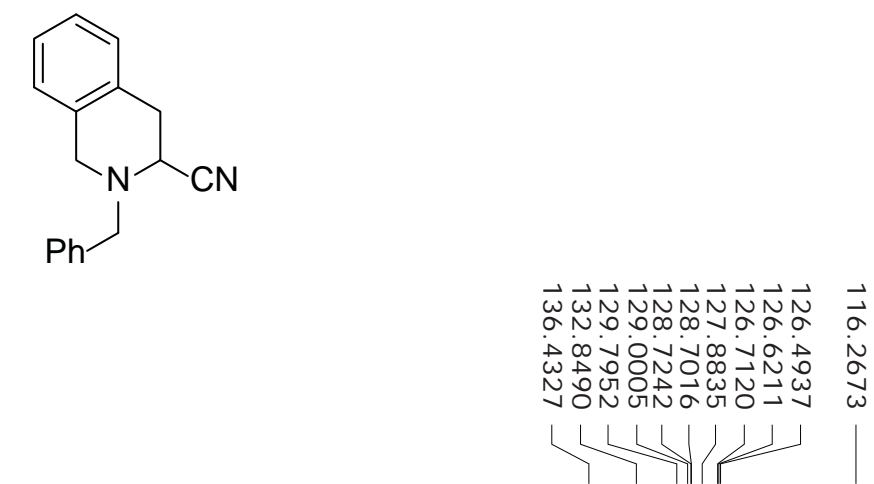

ㅊำ

जU:

जू०ष

|

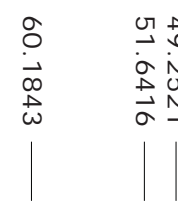

$w$
$\stackrel{w}{0}$
$\dot{\circ}$
บ
0

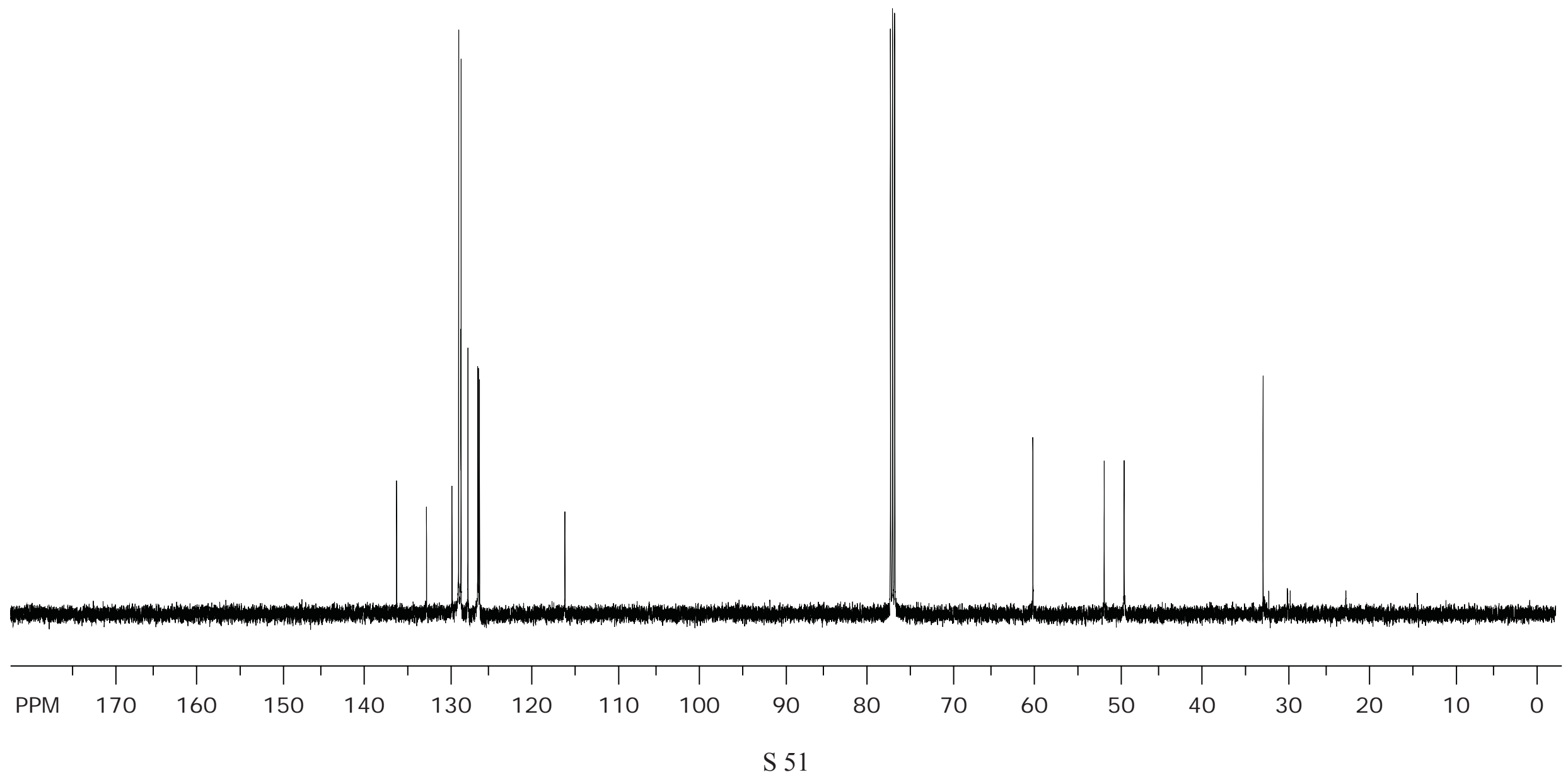

